\title{
Molecular Dynamics of Soft Wetting
}


Molecular Dynamics of Soft Wetting

Liz Mensink 


\section{Thesis committee members:}

Prof. dr. J. L. Herek (chairman) Universiteit Twente

Prof. dr. J. H. Snoeijer (promotor) Universiteit Twente

Dr. S. de Beer (co-promotor) Universiteit Twente

Prof. dr. U. Thiele

Universität Münster

Prof. dr. ir. J. T. Padding

Universiteit Delft

Prof. dr. S. Luding

Prof. dr. G. J. Vancso

Universiteit Twente

Dr. ir. W. M. de Vos

Universiteit Twente

Universiteit Twente
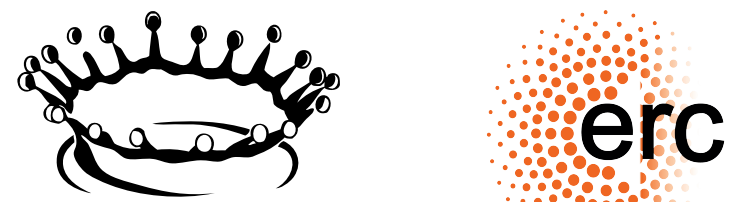

Physics of Fluids

The work in this thesis was carried out at the Physics of Fluids group of the Faculty of Science and Technology of the University of Twente. This thesis was financially supported by the European Research Council (ERC) Consolidator grant no. 616918.

Nederlandse titel:

Moleculaire Dynamica van bevochtiging op zachte oppervlakken

Front cover: Sjoukje Schoustra \& Liz Mensink

Publisher:

Liz Mensink, Physics of Fluids, University of Twente, P.O. Box 217, 7500 AE Enschede, The Netherlands pof.tnw.utwente.nl

Print: Gildeprint B.V., Enschede.

(C) Liz Mensink, Enschede, The Netherlands 2019.

No part of this work may be reproduced by print photocopy or any other means without the permission in writing from the publisher.

ISBN: 978-90-365-4839-7

DOI: $10.3990 / 1.9789036548397$ 


\section{MOLECULAR DYNAMICS OF SOFT WETTING}

\section{PROEFSCHRIFT}

ter verkrijging van

de graad van doctor aan de Universiteit Twente, op gezag van de rector magnificus,

Prof. dr. T.T.M. Palstra, volgens besluit van het College voor Promoties in het openbaar te verdedigen op vrijdag 11 oktober 2019 om 14:45 uur

door

Liz Ida Sien Mensink geboren op 5 juni 1991

te Oldenzaal, Nederland 
Dit proefschrift is goedgekeurd door de promotor:

Prof. dr. J. H. Snoeijer

en door de co-promotor:

Dr. S. de Beer 


\section{Contents}

1 Introduction 1

1.1 Matter: solids, liquids, gases and the in-between . . . . . . . . . 1

1.2 Bio-inspired soft matter research . . . . . . . . . . . . 2

1.3 Wetting of brushes $\ldots \ldots \ldots \ldots \ldots$

1.3 .1 Polymer brushes . . . . . . . . . . . . . . . . . . . . . . . . .

1.3 .2 Wetting and droplets . . . . . . . . . . . . . . 4

1.4 A macroscopic view on soft wetting . . . . . . . . . . . 6

1.4 .1 Wetting ridges . . . . . . . . . . . . . 6

1.4 .2 Surface tension and surface free energy . . . . . . . . 7

1.5 A guide through this thesis $\ldots \ldots \ldots \ldots$

2 Molecular Dynamics Simulations 13

2.1 Molecular Dynamics . . . . . . . . . . . . . . . . . . 13

$2.1 .1 \quad$ Interaction potentials . . . . . . . . . . . . . . . . . . . . . . . . . . .

2.1 .2 Ensembles . . . . . . . . . . . . . . . . . . . . . . . . 16

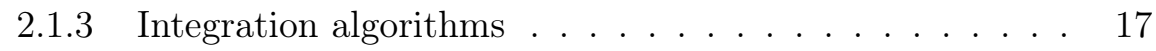

2.2 Observables and characterization $\ldots \ldots \ldots \ldots \ldots$

2.2 .1 Density, pressure and strain . . . . . . . . . . . . . 18

$2.2 .2 \quad$ Surface tension . . . . . . . . . . . . . . . . . . . . 19

2.2 .3 Contact angle . . . . . . . . . . . . . . . 20

2.2 .4 Overlap integrals and binary interactions . . . . . . 20

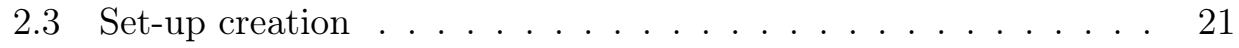

2.3 .1 Polymer brush $\ldots \ldots \ldots \ldots \ldots . \ldots \ldots 22$

2.3 .2 Polymer gel . . . . . . . . . . . . . . . . . . . 23

$\begin{array}{lll}3 & \text { Wetting of Polymer Brushes by Polymeric Nanodroplets } & 27\end{array}$

3.1 Introduction . . . . . . . . . . . . . . . . 27

3.2 Model and Methods . . . . . . . . . . . . . . . . . . 28

3.3 Results and Discussion . . . . . . . . . . . . . . . . . 30

3.4 Conclusions . . . . . . . . . . . . . . . . . . . . . . 36 
3.5 Appendix $\ldots \ldots \ldots \ldots \ldots$

$3.5 .1 \quad$ Transition regime $\ldots \ldots \ldots \ldots . \ldots . \ldots . \ldots$

3.5 .2 Wetting ridges . . . . . . . . . . . . . . . . . . . . 39

4 The role of Entropy in Wetting of Polymer Brushes 47

4.1 Introduction . . . . . . . . . . . . . . . . . . 48

4.2 Model and Methods . . . . . . . . . . . . . . . . . . . . . . 50

$4.2 .1 \quad$ Molecular Dynamics Simulations . . . . . . . . . . 50

4.3 Theory $\ldots \ldots \ldots \ldots \ldots \ldots \ldots$

4.3 .1 Mixing-Demixing . . . . . . . . . . . . . . . 53

$4.3 .2 \quad$ Complete to Partial Wetting . . . . . . . . . . . 56

4.4 Results and discussion . . . . . . . . . . . . . . . . . . . . 57

$4.4 .1 \quad$ Mixing - demixing . . . . . . . . . . . . . . 57

4.4 .2 Partial wetting - complete wetting . . . . . . . . . 61

4.4 .3 Partial wetting . . . . . . . . . . . . . . . . . . . . . . . . .

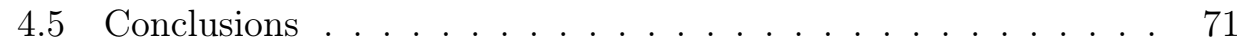

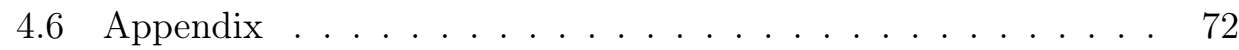

$4.6 .1 \quad$ Brush swelling . . . . . . . . . . . . . . . . . . 72

4.6 .2 Surface tensions. . . . . . . . . . . . . . . . . 72

5 Nanoscale elasto-capillary probes of the Shuttleworth effect 79

5.1 Introduction . . . . . . . . . . . . . . . . . . . . . . 79

5.2 Molecular Dynamics simulations $\ldots \ldots \ldots \ldots$

5.2 .1 Materials . . . . . . . . . . . . . . . . . . 81

5.2 .2 Configurations . . . . . . . . . . . . . . 83

5.3 Results . . . . . . . . . . . . . . . . . . . . . . . . . . 87

$5.3 .1 \quad$ Contact angles $\ldots \ldots \ldots \ldots \ldots$. . . . . . . . . . 87

$5.3 .2 \quad$ Elastic Wilhelmy plate . . . . . . . . . . . . . . . 88

5.3 .3 Direct measurement of surface tension . . . . . . . . . . 90

5.4 Discussion . . . . . . . . . . . . . . . . . . . . . . . . . . . . 93

$\begin{array}{lr}\text { Summary } & 97\end{array}$

$\begin{array}{ll}\text { Samenvatting } & 101\end{array}$

\begin{tabular}{ll}
\hline Acknowledgements & 105
\end{tabular} 


\section{Chapter 1}

\section{Introduction}

\subsection{Matter: solids, liquids, gases and the in-between}

For many centuries humankind has tried to create understanding of its surroundings by classifying them into different categories. For example, we classify living organisms into subcategories of bacteria, funghi, plants and animals. Likewise, we also organise matter into different categories, the most well-known of which are solids, liquids, and gases [1]. However, as one ventures deeper into the world of materials science, the boundaries between these substance classes become more vague as materials start to exhibit properties that cannot be allocated to one of the three most well-known categories. Examples of such materials one commonly encounters are for example the glass in window-panes, lightning bolts, silly putty and quicksand, but also liquid crystals used in LCD displays.

In the world of soft matter, these blurred boundaries becomes more apparent, as soft materials share properties of both the traditional solids and traditional liquids, thus forming a sub-category of its own. This subcategory
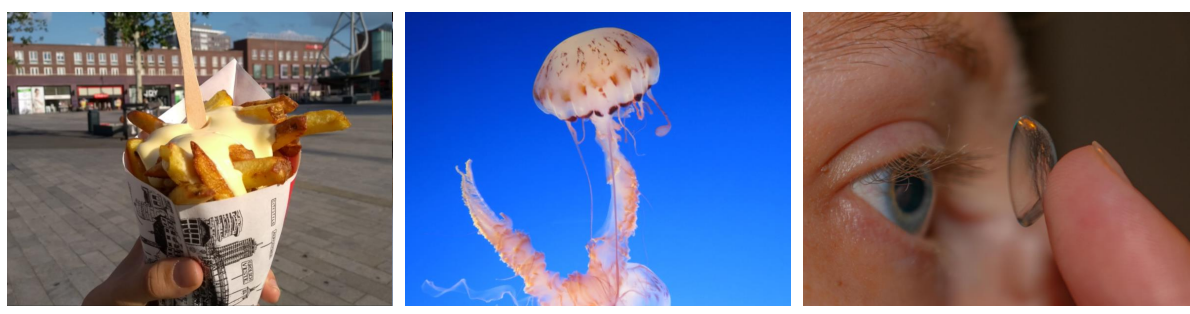

Figure 1.1: Examples of soft condensed matter: (a) Mayonnaise, (b) a jellyfish, and (c) a contact lens. 
comprises of many different materials, such as liquid crystals, foams, gels, polymers and granular materials [2], as is illustrated in figure 1.1. Many examples of soft matter can be found in biology, where many organisms consist of or use soft materials to their advantage.

Where solids such as metals and ceramics are considered to be nearly perfectly rigid, soft solids are capable of undergoing very large deformations without losing their structural integrity. In this thesis, we will further explore the properties of such soft solids. Specifically, we will look at the interactions between such soft, deformable surfaces and liquids, i.e. wetting phenomena.

\subsection{Bio-inspired soft matter research}

Nature has been a source of inspiration and fascination to many researchers, as nature has developed its own solutions to many of our modern-day challenges. A lot of these solutions are found inside living systems and their use of soft matter. A plethora of examples of soft matter used in clever ways in nature exists, such as the water-repellent lotus leaves, to hairy structures in ears and nose that catch and transport pollutants.

In turn, research is finding its way to incorporate soft matter to serve new purposes. For example, by developing an adhesive that directly mimics the glue-like properties of mussels and gecko feet [3]. A schematic to this approach is shown in figure 1.2(a), in which the gecko feet were used as an inspiration for structured substrates, which was then covered by polydopamine, a polymer that is found in the mussels' byssal threads. Other research has focused on creating surface coatings that are nonbiofouling, meaning that no proteins or cells will adhere to the surface [4. Several approaches to this challenge have arisen [5, 6], using either micropatterned surfaces, or polymer coatings to alter the wetting properties of the surface. Micropatterned surfaces with pillar-like structures are found in nature on lotus-leaves, to keep them water-repellent. Likewise, animals also show such patterned surfaces, the water-strider for example, can stand and stride on water due to an intricate needle-like structure on its legs that help repel water [7]. One last useful application we will discuss, uses brush-like structures as are also found inside the human body in for example lungs and intestines, where they aid in the selective absorption of substances into the body [9, 10]. In this last application [8, 11], cotton sheets are covered in such a polymer brush, to capture moisture from cool air, which is then released upon heating by thermo-activation of the polymer brush. A schematic of this thermo-activation is pictured in figure 1.2 (b), showing extended brush structures catching water vapor, then collapsing and releasing water under the influence of light, through a chemical alteration. This moisture-catching cloth 
(a)
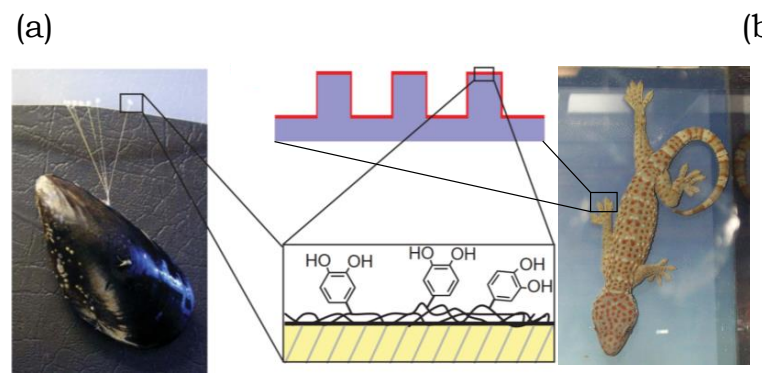

(b).

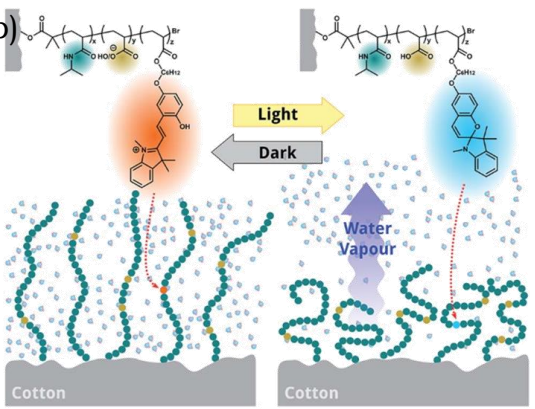

Figure 1.2: Examples of recent advances in soft materials: (a) A schematic showing the building blocks of a reversible adhesive, inspired by mussels and geckos. Image adapted from Lee et al. [3] with permission. (b) Light-activated water uptake and expulsion in polymer-covered cotton fibers. The left image shows and extended, moisture-capturing brush in the dark. The right image shows the same brush collapsed, releasing the captured moisture in the light. Image reproduced from ter Schiphorst et al. [8] with permission.

can for example serve as a way to collect water in desertous areas. In these last few examples, the wetting interactions between the soft matter and a solvent play an important role. The fundamental knowledge obtained in this thesis, will aid the further development of these applications.

\subsection{Wetting of brushes}

\subsubsection{Polymer brushes}

Many interesting applications, such as the fog-catching cotton sheets, involve the interaction of liquids in contact with a polymer brush. Polymer brush is the term used to describe the brush-like structure that arises when long polymer chains are grafted densely together onto a rigid substrate, as illustrated in figure 1.3(a). Important parameters of the brush are the polymer chain length $N_{\mathrm{B}}$, and the grafting density $\sigma_{\mathrm{GD}}$ which is defined by dividing the number of polymers $\sigma_{\mathrm{p}}$ by the square area $a^{2}, \sigma_{\mathrm{GD}}=\frac{\sigma_{\mathrm{p}}}{a^{2}}$. If the size of a free polymer coil in a bulk of solvent is smaller than the grafting density, this is referred to as a low grafting density. In such a case the polymers attached to the substrate, coil up into structures that are referred to as 'pancakes' or 'mushrooms' [12]. For higher grafting densities, polymer brushes are attained, due to sterical hindrance that forces the polymer chains to fan outward, resulting in a substrate that can be soft and deformable [13 16]. The conformations of these polymer structures are depicted in figure 1.3(a). Besides the grafting density, the 
(a)
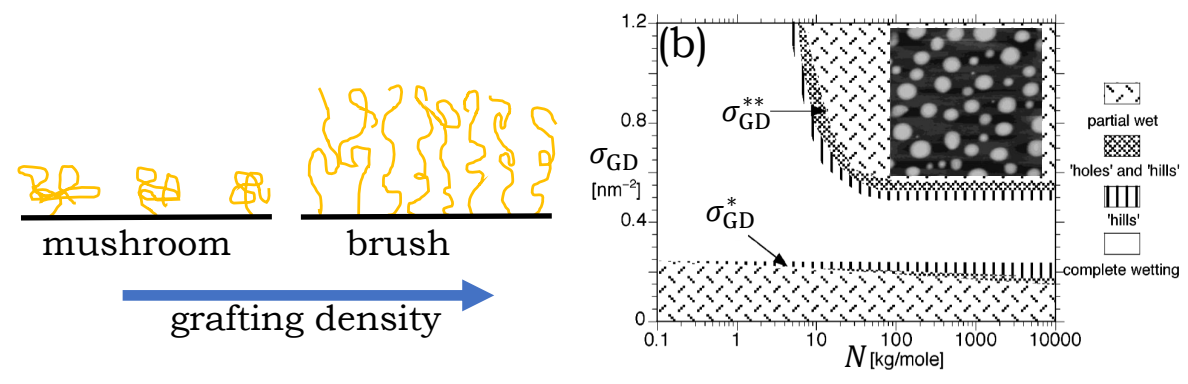

Figure 1.3: (a) Schematic showing the conformations of end-tethered polymer chains on a surface, using different grafting densities. Shown are mushrooms (left), and brushes (right). (b) Wetting phase diagram of a polystyrene melt wetting a polystyrene brush. $N$ denotes the number of polystyrene melt beads per chain. $\sigma_{\mathrm{GD}}$ is the brush grafting density. The inset shows an AFM image of the autophobic wetting phase. Adapted with permission from [20]. Copyright 2019 American Chemical Society.

presence of solvent also plays a key role in the structure of the polymer brush itself. In the polymer field solvents are divided into three categories: good, bad and $\theta$ solvents [17-19]. The solvent directly influences the degree of swelling in a polymer brush. A good solvent causes a brush to fan out more, meaning that the brush 'prefers' to interact with the solvent over interactions with other brushes. A bad solvent on the contrary causes the brush to become more collapsed, as the brush now prefers to interact with itself over the solvent. A $\theta$-solvent here is a special case in which the solvent is exactly at the transition point from a poor to a good solvent. In such a solvent, polymer chains act like freely-jointed chains, neglecting any interactions with other monomers in the chain.

\subsubsection{Wetting and droplets}

A bulk phase of a liquid solvent can, besides influencing the degree of swelling of the brush, also give rise to different wetting phases when interacting with a polymer brush. Already in the simplest case, where the brush is wetted by an identical polymer melt, one encounters highly unexpected autophobic wetting behavior 20, for which an identical polymer melt does not wet its identical polymer brush substrate. Instead, a droplet of a finite contact angle will form on such a substrate. The wetting regimes for a polystyrene brush wetted by a polystyrene melt, as reported by Maas et al. [20] are given in figure $1.3(\mathrm{~b})$. In this figure, the grafting density $\sigma_{\mathrm{GD}}$ is plotted against the degree of polymerization of the identical polymer melt $N$. The different phases 


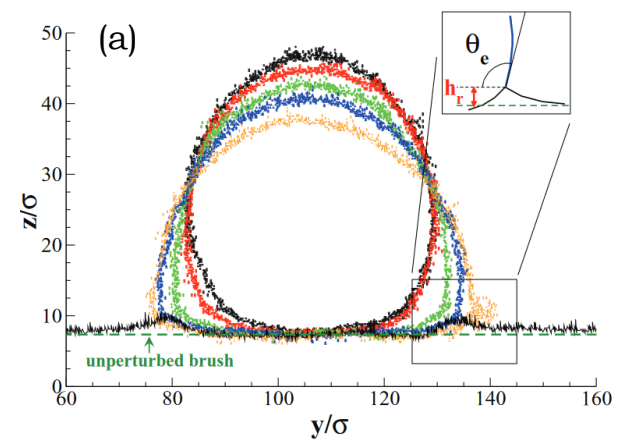

(b)

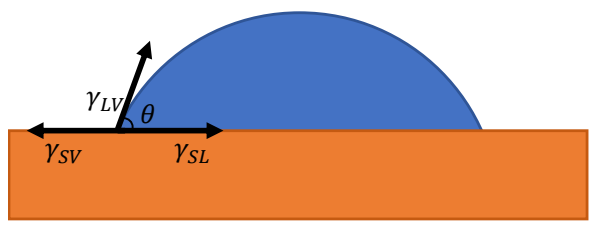

Figure 1.4: (a) Wetting ridges occuring in a polymer brush wetted by a polymer melt. Image reproduced from Léonforte and Müller [21] with permission. Shown are the isodensity contours for droplets and brush at an increasing wettability (black to orange). (b) A droplet wetting a rigid substrate. Drawn in the figure are the surface tensions $\gamma_{\mathrm{SV}}, \gamma_{\mathrm{LV}}$ and $\gamma_{\mathrm{SL}}$ acting along the interfaces, and the resulting contact angle $\theta$.

are denoted in the legend, where partial wetting indicates that the material is autophobic. The plot also shows two intermediate regimes, denoted using 'hills' and 'holes and hills'. In these cases, a combination of partial and complete wetting, and partial, complete and non-wetting states are found. The upper right part of the phase diagram shows that, in the case of a densely grafted brush wetted by a melt of long chains, the melt becomes unable to mix with the densely grafted polymer brush due to entropic reasons. An autophobic regime was also reached for low grafting densities, caused by the polymer melt interacting with the substrate below the polymer brush. In other cases away from the boundaries, complete wetting was observed.

Given that droplets can form, we should also consider the interactions between liquid droplets and a brush. An example of this is given in figure 1.4(a), showing density contours of a droplet wetting a polymer brush, with an increasing degree of wettability [21]. The figure clearly reveals the presence of a wetting ridge located underneath the three-phase contact line.

Besides brushes, it is also of interest to consider other types of soft substrates that have a very different molecular structure. For example, a polymer gel is a more homogeneously structured material than a polymer brush, consisting of a network of polymers that are either physically or chemically bonded together. A classical example of a gel is the famous gelatin pudding, in which gelatin forms a network that is capable of containing many times its own weight in water. Such elastomeric polymer gels, like polymer brushes, also display autophobicity, for similar entropic reasons [22, 23]. 
This structural difference raises the question whether wetting behavior is universal for all soft materials. For this, we now take a step back and consider a more macroscopic view on the wetting interactions.

\subsection{A macroscopic view on soft wetting}

The key macroscopic characteristic of wetting is the contact angle $\theta$ that a drop makes with respect to the substrate (see figure 1.4(b)). On a solid that is perfectly rigid, this angle is given by Young's law:

$$
\gamma_{\mathrm{SV}}=\gamma_{\mathrm{SL}}+\gamma_{\mathrm{LV}} \cos (\theta)
$$

Here $\gamma$ is the surface tension, and the subscripts S, V and L refer to solid, vapor and liquid, thus denoting the two phases that are separated by the interface. Young's law describes a macroscopic force balance in the horizontal direction. We have learned from the previous paragraph, however, that soft solids exhibit an interesting departure from these traditional wetting studies, in the form of a wetting ridge. It becomes apparent that the surface tension forces pull on the substrate (see also the vertical force component in figure 1.4(b)), which for very soft substrates results in a visible elastic deformation. Below we describe recent progress on the wetting on substrates that are characterized by an elastic Young's modulus $G$.

\subsubsection{Wetting ridges}

The wetting behavior can be divided into three regimes [24], depending on the elastocapillary length $\gamma / G$, where $\gamma$ is the surface free energy, and $G$ the Young's modulus of the material. This length needs to be compared to the range of the molecular interactions $a$, and the droplet size $R$, to determine the wetting regime.

In the first regime, where the capillary length is smaller than the range of the molecular interactions, $\gamma / E \ll a$, the surface deformation is negligible and Young's law applies. This is shown in figure 1.5(a). The second regime occurs when the elastocapillary length is larger than the molecular interactions, but small compared to the droplet size, $a \ll \gamma / G \ll R$. This is when wetting ridges are formed at a scale $\gamma / G[25,26$, yet Young's law holds at the macroscopic scale, as corresponding to figure 1.5(b). Lastly, for cases where $\gamma / G \gg R$ the macroscopic contact angles will also deviate from predictions by Young's law, and start to approach Neumann's law for liquid-liquid wetting instead [27, 28], as shown in figure 1.5(c). Note that in soft solids the elastic modulus can go as low as $1 \mathrm{kPa}$ or lower, meaning that the wetting ridge can reach up to 10-100 


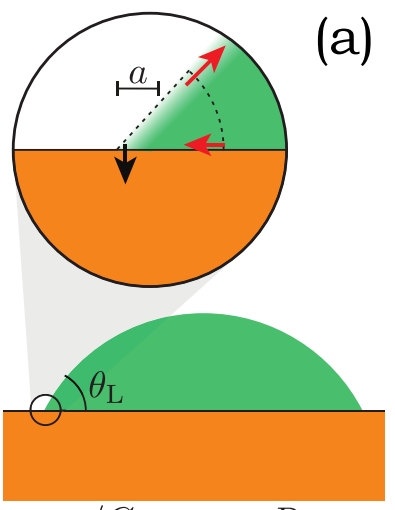

$\gamma / G \ll a \ll R$

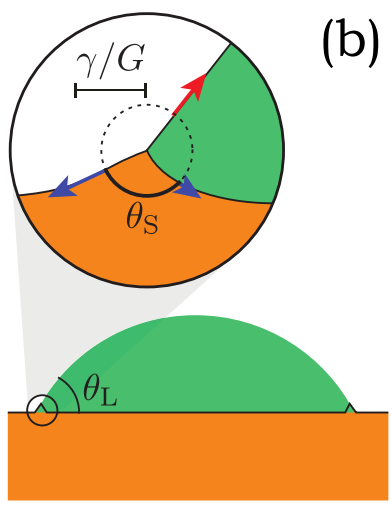

$a \ll \gamma / G \ll R$

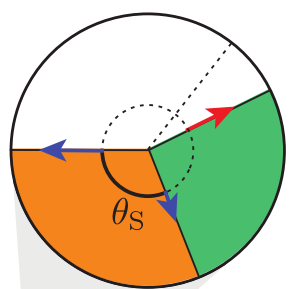

(c)

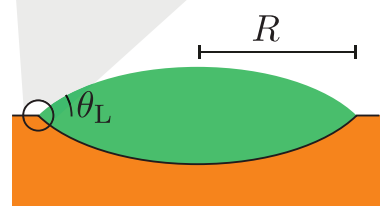

$a \ll R \ll \gamma / G$

Figure 1.5: Influence of the elastocapillary length $\gamma / G$ on wetting behavior (image adapted from Andreotti and Snoeijer, to appear in Annual Review Fluid Mechanics 2020 [24]). The panels correspond to different values of the elastocapillary length, with respect to the range of molecular interactions $a$, and the drop size $R$.

microns, values that can easily be detected experimentally. Thus, a low elastic modulus combined with the forces near an interface allow the wetting behavior for soft materials to be very different from more rigid solids.

\subsubsection{Surface tension and surface free energy}

We have shown that the surface energy leads to a force pulling along the interface between substances, and that wetting ridges can arise as a result. For liquids of constant composition and at a constant temperature, this surface energy takes on a constant value. For solids, however, the surface energy depends on the elastic strain on the solid, denoted as $\epsilon$, so that $\gamma(\epsilon)$. This implies that the contact angle of droplets on solids can depend on strain.

For this implication, we refer to experiments showing that the contact angles of droplets can indeed change for strained solids [29]. The results of this experiment are shown in figure 1.6. They report a significant change in the contact angle for a strained polycarbonate glass, in which the strained solid is more hydrophilic (figure 1.6(a)). However, no change was observed for the wetting angle of an elastomer (figure 1.6(b)). According to Young's law, equation 1.1, this indeed implies a strain-dependence of $\gamma_{\mathrm{SV}}-\gamma_{\mathrm{SL}}$ for the glassy substrate.

Interestingly, whether or not the wetting behavior changes in strained elastomers, is still debated. Other researchers have reported a significant change in the solid angle for strained elastomers [30, 31], pointing towards a significant 


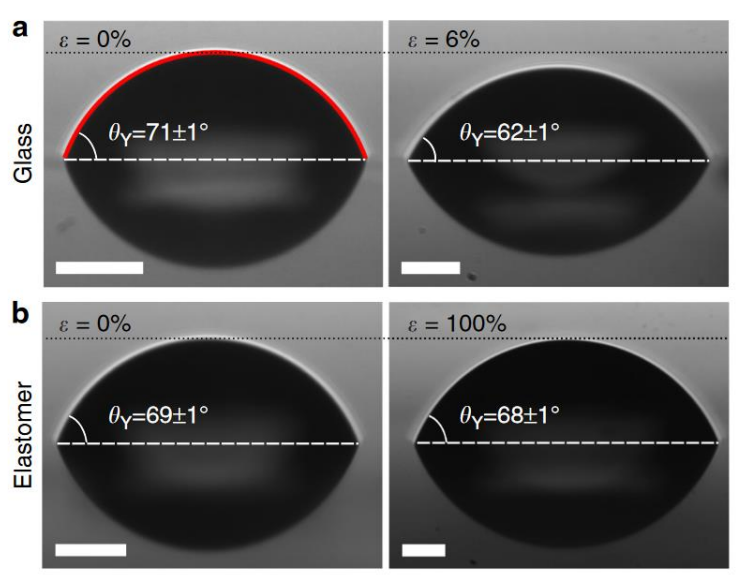

Figure 1.6: Contact angle measurements of a droplet wetting a polycarbonate glass (a) and an Elastollan elastomer (b). Pictures on the left side show the contact angles $\theta_{\mathrm{Y}}$ of a droplet wetting the unstrained solid. The right side shows the contact angles of droplets wetting a strained solid. The strain is indicated using $\epsilon$. Reprinted from Schulman et al. [29], CC-BY 4.0.

change in surface tensions.

A consequence of this strain dependence, is that the surface free energy and the surface tension are not equal. To make the difference more apparent, we will look at the respective definitions for the surface energy and the surface tension. The surface energy is defined by a lack of intermolecular bonds that occurs near a surface. This leads to an excess free energy per area, due to the presence of an interface, with units of $\mathrm{J} / \mathrm{m}^{2}$.

The surface tension is defined by the amount of (reversible) work that is needed to increase the area of an existing interface. It is the force per unit length, located in the interfacial region, meaning that this has a mechanical definition, with units of $\mathrm{N} / \mathrm{m}$. Therefore, the physical meaning of these quantities is different and in the case of soft solids, not necessarily identical [32]. These two quantities are related to one another by the Shuttleworth equation 33]:

$$
\Upsilon=\gamma+d \gamma / d \epsilon
$$

This equation also immediately shows why this distinction becomes only important in solids, as liquids do not have a reference state. This relation opens up questions such as how strong this Shuttleworth effect is, how it affects soft wetting behavior, and how the Shuttleworth effect can be measured. 


\subsection{A guide through this thesis}

In this thesis we use Molecular Dynamics to study the wetting behavior of two distinct soft solid structures, namely brushes and gels.

In chapter 2, we will first expand upon the basic principles of Molecular Dynamics, as well as analysis methods that are important to understanding the behavior and material properties from simulated soft solids.

In chapter 3 we explore the behavior of a polymer droplet wetting a polymer brush for a wide range of interactions. This chapter shows a remarkable difference in wetting behavior between the different kinds of soft solids, in which increasingly softer polymer brushes show increasingly hydrophobic wetting behavior. This is in contrast to the wetting of soft gels, where the softness induces hydrophilicity.

In chapter 4 we keep our focus on the wetting behavior of polymer brushes, where we now vary the grafting density of the polymer brush, the chain length of the polymer melt droplet, as well as the wettability of the polymer brush. We then study the effects of entropy on the wetting behavior of polymer brushes, by comparing our results to theories derived for chemically identical brushes and melts.

In chapter 5 we present a comparison of a soft gel to a nearly rigid solid, focusing on the effect of strain on the wetting behavior. This chapter further explores the Shuttleworth effect, which states that the surface free energy of a solid changes as it is put under strain. More specifically, we demonstrate multiple ways in which the Shuttleworth effect can be characterized, and we will quantify the strength of the Shuttleworth effect for model substrates.

\section{Bibliography}

[1] M. de Podesta, Understanding the Properties of Matter (UCL Press, London) (1996).

[2] D. M. Jones, J. R. Smith, W. T. S. Huck, and C. Alexander, "Variable adhesion of micropatterned thermoresponsive polymer brushes: Afm investigations of poly(n-isopropylacrylamide) brushes prepared by surfaceinitiated polymerizations", Adv. Mater. 14, 1130 (2002).

[3] H. Lee, B. P. Lee, and P. B. Messersmith, "A reversible wet/dry adhesive inspired by mussels and geckos", Nature 448, 338 (2007).

[4] A. M. Brzozowska, F. J. Parra-Velandia, R. Quintana, Z. Xiaoying, S. S. C. Lee, L. Chin-Sing, D. Jańczewski, S. L.-M. Teo, and J. G. Vancso, 
"Biomimicking micropatterned surfaces and their effect on marine biofouling", Langmuir 30, 9165-9175 (2014), URL https://doi.org/10.1021/ 1a502006s pMID: 25017490.

[5] W. J. Yang, T. Cai, K.-G. Neoh, E.-T. Kang, G. H. Dickinson, S. L.-M. Teo, and D. Rittschof, "Biomimetic anchors for antifouling and antibacterial polymer brushes on stainless steel", Langmuir 27, 7065-7076 (2011).

[6] V. B. Damodaran and N. S. Murthy, "Bio-inspired strategies for designing antifouling biomaterials", Biomaterials Research 20 (2016).

[7] X. Gao and L. Jiang, "Water-repellent legs of water striders", Nature 432, 36 (2004).

[8] J. ter Schiphorst, M. van den Broek, T. de Koning, J. N. Murhpy, A. P. H. J. Schenning, and A. C. C. Esteves, "Dual light and temperature responsive cotton fabric functionalized with a surface-grafted spiropyrannipaam-hydrogel", Journal of Materials Chemistry A 4 (2016).

[9] I. Szleifer, "Polymers and proteins: interactions at interfaces", Current Opinion in Solid State and Materials Science 2, 337 - 344 (1997), URL http://www.sciencedirect.com/science/article/pii/ S1359028697801258.

[10] L. M. Ensign, R. Cone, and J. Hanes, "Oral drug delivery with polymeric nanoparticles: The gastrointestinal mucus barriers", Advanced Drug Delivery Reviews 64, 557 - 570 (2012), URL http://www. sciencedirect. com/science/article/pii/S0169409X11003036, advances in Oral Drug Delivery: Improved Biovailability of Poorly Absorbed Drugs by Tissue and Cellular Optimization.

[11] H. Yang, H. Zhu, M. M. R. M. Hendrix, N. J. H. G. M. Lousberg, G. de With, A. C. C. Esteves, and J. H. Xin, "Temperature-triggered collection and release of water from fogs by a sponge-like cotton fabric", Advanced Materials 25, 1150-1154 (2013).

[12] W. J. Brittain and S. Minko, "A structural definition of polymer brushes", Polym. Sci., Part A: Polym. Chem. 45, 3505 (2007).

[13] P. G. de Gennes, "Conformations of polymers attached to an interface", Macromolecules 13, 1069 (1980).

[14] P. G. de Gennes, "Polymers at an interface, a simplified view", Advances in Colloid and Interface Science 27, 189-209 (1987). 
[15] S. T. Milner, T. A. Witten, and M. E. Cates, "Theory of the grafted polymer brush", Macromolecules 21, 2610 (1988).

[16] M. Murat and G. S. Grest, "Interaction between grafted polymeric brushes: A molecular-dynamics study", Phys. Rev. Lett. 63, 1074 (1989).

[17] F. Brochard and P. G. de Gennes, "Dynamical scaling for polymers in theta solvents", Macromolecules 10, 1157-1161 (1977), URL https:// doi.org/10.1021/ma60059a048.

[18] G. S. Grest and M. Murat, "Structure of grafted polymeric brushes in solvents of varying quality: a molecular dynamics study", Macromolecules 26, 3108-3117 (1993), URL http://dx.doi.org/10.1021/ma00064a019.

[19] A. Karim, S. K. Satija, J. F. Dougla, J. F. Ankner, and L. J. Fetters, "Neutron reflectivity study of the density profile of a model end-grafted polymer brush: Influence of solvent quality", Physical Review Letters 73, 3407-3410 (1994).

[20] J. H. Maas, G. J. Fleer, F. A. M. Leermakers, and M. A. Cohen Stuart, "Wetting of a polymer brush by a chemically identical polymer melt: Phase diagram and film stability", Langmuir 18, 8871-8880 (2002).

[21] F. Léonforte and M. Müller, "Statics of polymer droplets on deformable surfaces", The Journal of Chemical Physics 135, 214703 (2011), URL https://doi.org/10.1063/1.3663381.

[22] T. Kerle, R. Yerushalmi-Rozen, and J. Klein, "Cross-link-induced autophobicity in polymer melts: A re-entrant wetting transition", Europhysics Letters (EPL) 38, 207-212 (1997), URL https://doi.org/10. 1209\%2Fepl\%2Fi1997-00226-8.

[23] J. Jopp and R. Yerushalmi-Rozen, "Autophobic behavior of polymers at the melt-elastomer interface", Macromolecules 32, 7269-7275 (1999), URL https://doi.org/10.1021/ma990555i.

[24] B. Andreotti and J. H. Snoeijer, "Statics and dynamics of soft wetting", Annual Review of Fluid Mechanics (2020).

[25] E. R. Jerison, Y. Xu, L. A. Wilen, and E. R. Dufresne, "Deformation of an elastic substrate by a three-phase contact line", Phys. Rev. Lett. 106, 186103 (2011), URL https://link.aps.org/doi/10.1103/ PhysRevLett.106.186103. 
[26] L. A. Lubbers, J. H. Weijs, L. Botto, S. Das, B. Andreotti, and J. H. Snoeijer, "Drops on soft solids: free energy and double transition of contact angles", Journal of Fluid Mechanics 747, R1-12 (2014).

[27] A. Marchand, S. Das, J. H. Snoeijer, and B. Andreotti, "Contact angle on a soft solid: From young's law to neumann's law", Physical Review Letters 109 (2012).

[28] R. W. Style and E. R. Dufresne, "Static wetting on deformable surfaces, from liquids to soft solids", Soft Matter 8, 7177-7184 (2012).

[29] R. D. Schulman, M. Trejo, T. Salez, E. Raphael, and K. Dalnoki-Veress, "Surface energy of strained amorphous solids", Nature Communications 9, 982 (2018).

[30] Q. Xu, K. Jensen, R. Boltyanskiy, R. Sarfat, R. W. Style, and E. R. Dufresne, "Direct measurement of strain-dependent solid surface stress", Nature Communications 8, 555 (2017).

[31] H. Liang, Z. Cao, Z. Wang, and A. V. Dobrynin, "Surface stresses and a force balance at a contact line", Langmuir 34, 7497-7502 (2018), URL https://doi.org/10.1021/acs.langmuir.8b01680

[32] R. W. Style, A. Jagota, C.-Y. Hui, and E. R. Dufresne, "Elastocapillarity: Surface tension and the mechanics of soft solids", Annual Review of Condensed Matter Physics 8 (2016).

[33] R. Shuttleworth, "The surface tension of solids", Proceedings of the Physical Society. Section A 63 (1950). 


\section{Chapter 2}

\section{Molecular Dynamics Simulations}

Molecular Dynamics (MD) simulations are a powerful tool in obtaining a microscopic understanding of the physical and chemical properties of materials. $M D$ is a type of many-body computer simulation, in which many particles are placed inside a simulation box. By defining specific interaction potentials between the particles and solving Newton's equations of motion, the particles behave similar to real-life atoms and molecules. From the velocities and positions of the particles, information such as the density, the stresses and the temperature can be calculated. In order to transform the results to macroscopic observables, great care must be taken to perform the coarse-grained averaging. Since throughout this thesis MD will be the central tool, we dedicate this chapter to summarizing the main principles of $M D$ and to describing specific methods of analysis used in this thesis for the wetting of soft materials.

\subsection{Molecular Dynamics}

In MD simulations, Newton's equation of motion, $\vec{F}_{i}=m \overrightarrow{a_{i}}$, is solved for a specified set of particles, each of which is labelled with index $i$. When performing a MD simulation, the first step is to define the positions and interactions of particles, and input parameters such as the time step and total simulation steps. Then the main part of the MD simulation begins: The forces on all particles are calculated. From this data, the positions and velocities of the particles are updated using Newton's equations. This process is repeated until the end of the simulation time is reached (see also figure 2.1). Computing the forces on all particles is the part of a MD simulation that consumes the most time. In order to save on this time, several optimization techniques have been 


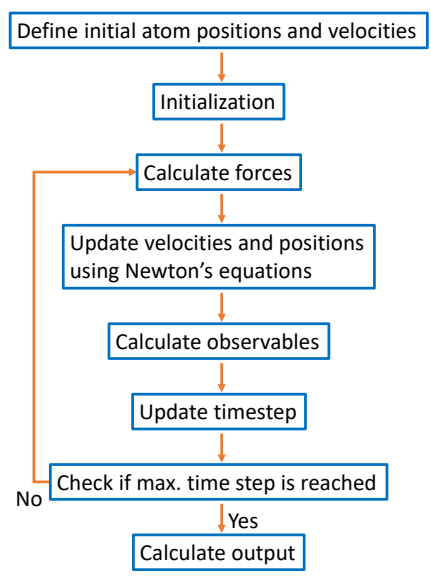

Figure 2.1: A flow-chart of a typical Molecular Dynamics algorithm.

developed over time for MD simulations. For detailed descriptions, we refer to the textbook 'Understanding molecular simulation' by D. Frenkel and B. Smit [1].

The process of setting up a MD simulation is in many aspects similar to performing experiments. In the first stage, the set-up is built and the system is equilibrated for a certain number of time steps. After equilibrating, the actual measurement is started, where simulations run for a certain time and the desired data is collected over this time. In MD simulations it is very important to gather sufficient statistics, as statistical mechanics form the connection from microscopic MD simulations to macroscopic properties. The gathered statistics are needed to average out noise in order to obtain an accurate measurement of macroscopic observables that will be described in section 2.2.

\subsubsection{Interaction potentials}

An important part of the simulation of realistic material properties, lies in the choice of a proper interaction potential. This interaction potential aims to approximately describe the interaction between a pair of particles. Perhaps the most well-known interaction is the Lennard-Jones potential [2]:

$$
V_{\mathrm{LJ}}\left(r_{i j}\right)=4 \epsilon\left(\left(\frac{\sigma}{r_{i j}}\right)^{12}-\left(\frac{\sigma}{r_{i j}}\right)^{6}\right),
$$

where $r_{i j}$ is the distance between particles $i$ and $j, \epsilon$ is the interaction strength and $r=\sigma$ is the zero-crossing of the potential This potential describes the behavior between two neutral particles (for example in noble gases). These 


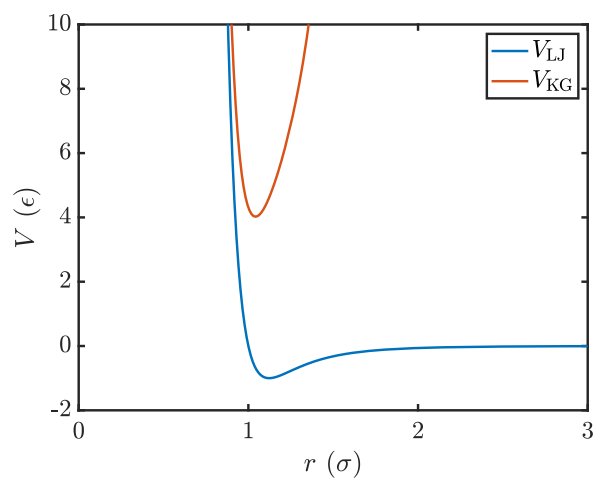

Figure 2.2: Depiction of the intermolecular potential between nonbonded particles $\left(V_{\mathrm{LJ}}\right)$ and the bonded particles $\left(V_{\mathrm{KG}}\right)$.

particles will feel a long-range attractive van der Waals force, and a short range repulsive force that is known as Pauli repulsion. As the long-range attraction between particles approaches zero, a cut-off length $r_{\text {cut }}$ is used to set the potential to 0 for $r>r_{\text {cut }}$, so that only potentials are calculated for distances within the cut-off range $\left(r<r_{\text {cut }}\right)$.

A second interaction potential that is important in this thesis, is the Finitely Extensible Nonlinear Elastic (FENE) potential [3]. This potential is commonly used to describe the behavior of long-chained polymers, by simplifying the polymer to a sequence of beads connected by non-linear springs. It is defined as:

$$
V_{\mathrm{FENE}}\left(r_{i j}\right)=-0.5 K R_{0}^{2} \ln \left(1-\left(\frac{r}{R_{0}}\right)^{2}\right),
$$

where $K$ is the spring potential of the bond, and $R_{0}$ is the maximum extension of the bond. In order to simulate polymers in MD, Kremer and Grest [4 have combined the FENE potential with the LJ potential, to create a model that simulates polymer behavior:

$$
V_{\mathrm{KG}}\left(r_{i j}\right)=-0.5 K R_{0}^{2} \ln \left(1-\left(\frac{r}{R_{0}}\right)^{2}\right)+4 \epsilon\left(\left(\frac{\sigma}{r_{i j}}\right)^{12}-\left(\frac{\sigma}{r_{i j}}\right)^{6}\right)+\epsilon .
$$

The Lennard-Jones potential acts up to a cut-off distance $r_{\mathrm{c}}=2^{1 / 6} \sigma$. The finite extensibility of this potential prevents the crossing of bonds through two bonded beads so that the beads will remain bonded. We will refer to this potential as the Kremer-Grest potential. Figure 2.2 shows both the LennardJones potential and the Kremer-Grest potential.

Likewise, other interaction potentials exist to describe more specific interactions, such as for example electrostatic interactions, or chemical bonds 


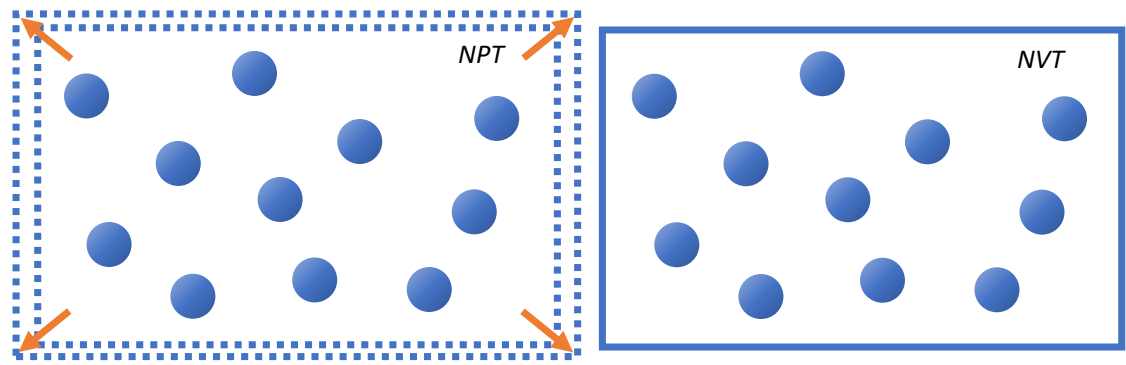

Figure 2.3: The two ensembles used for the MD simulations in this thesis. The dashed lines in the NPT ensembles show the variable system size.

at an angle. In the scope of this thesis however, we are solely interested in generic interactions and soft wetting behavior that can be described using the Lennard-Jones and FENE potentials.

When dealing with multiple species, we need to characterize the relative interaction between different particles. For this, we introduce the interaction parameter as defined by the Flory-Huggins solution theory [5]. This interaction parameter describes the interaction energy between two interacting phases (usually a polymer and a solvent). It is defined as:

$$
\chi=\frac{-z}{2} \frac{\left(\epsilon_{\mathrm{PP}}+\epsilon_{\mathrm{SS}}-2 \epsilon_{\mathrm{PS}}\right)}{k_{\mathrm{B}} T},
$$

where $z$ is the average number of neighbors per molecule, or the lattice coordination number, $\epsilon_{i j}$ is the interaction energy between two molecules. In this formula, the subscripts $\mathrm{P}$ and $\mathrm{S}$ refer to polymer segments and solvent molecules, respectively. This parameter describes the net affinity between two materials, and is useful to predict whether or not a material will mix.

\subsubsection{Ensembles}

Most experiments are performed while keeping certain parameters constant, such as temperature, pressure or volume. Likewise, in MD simulations, certain parameters can be kept constant to approach the desired system, using an ensemble [6]. The ensembles used in the MD simulations in this thesis are the $N V T$ and NPT ensembles. In both cases, the amount of particles $N$ and the temperature $T$ are kept constant. In $N V T$ ensemble the volume $V$ is also kept constant, whereas in the NPT ensemble the pressure $P$ is held at a constant value. A schematic of these ensembles is given in figure 2.3 .

Given that $T$ is held fixed, energy can be exchanged with the surroundings. In order to regulate temperature in an $N V T$ ensemble, several thermostats 
have been developed, such as the Berendsen [7], Andersen [8], Langevin and Nosé-Hoover [9] thermostats. These thermostats adapt the kinetic energy or velocities of the particles, to maintain or create the desired temperature.

The first method to adapt the particle velocities is through direct rescaling of velocities, as is done in the Berendsen thermostat and in velocity-rescaling. A second method to control temperature is by adding a stochastic force or velocity to the particles. This method is used by both the Langevin and Andersen thermostats, where the first applies a stochastic force, and the latter a velocity. Lastly, one can control the temperature by extending the equations of motion with an extra degree of freedom that acts as a thermal reservoir, using the Nosé-Hoover thermostat.

For MD simulations to reach equilibrium, the Langevin thermostat is useful. In this thermostat all particles experience a constant force of friction, and they receive a random force every timestep. The force and friction a particle experiences, are related so that the fluctuation-dissipation theorem is obeyed. This Langevin thermostat is generally used for complicated systems with long relaxation times, such as polymeric simulations. The Nosé-Hoover thermostat on the other hand, takes longer to reach an equilibrium state. At equilibrium however, this thermostat is considered to be the most reliable for simulations. A limitation to the aforementioned thermostats is that particles do not maintain their momentum, so they do not capture non-equilibrium hydrodynamics. As we will focus on systems at equilibrium however, other thermostats fall outside of the scope of this thesis.

Likewise, there are also several barostats to keep the pressure constant in an NPT ensemble. By scaling the inter-particle distances, the pressure in a system can be kept constant. An example of such a barostat is the ParinelloRahman barostat [10]. This barostat uses extended dimensions like the NoséHoover thermostat, but applied to pressure instead of temperature.

\subsubsection{Integration algorithms}

Various numerical algorithms are used to efficiently integrate Newton's equations. It is important that the algorithm remains accurate for 'large' timesteps $\Delta t$, and remains stable for long measurement times. Examples of such integrators are the leap-frog and Verlet algorithms [1. These algorithms all approximate the positions, velocities and accelerations of particles using linearizations. In MD simulations the velocity Verlet algorithm is commonly used, since it is a method where the velocity $v$ and the distance $x$ can be determined independently from one another. Furthermore, the accuracy is of third order in $x$ and second order in $v$, while requiring few steps for calculating.

The Verlet algorithm [11] determines the position of a particle at a certain 
moment in time, by taking into account the positions of the particle at one time unit forward in time, but also one time unit backward in time:

$$
\frac{\Delta^{2} x_{\mathrm{n}}}{\Delta t^{2}}=\frac{\frac{x_{\mathrm{n}+1}-x_{\mathrm{n}}}{\Delta t}-\frac{x_{\mathrm{n}}-x_{\mathrm{n}-1}}{\Delta t}}{\Delta t} .
$$

In the Verlet algorithm however, the velocities of particles are not directly generated, which is needed to compute the kinetic energy. For such an algorithm one would need a starting velocity values, which can be implemented. The velocity Verlet algorithm [1] is a version of the Verlet algorithm, where the velocity and position of a particle are calculated at the same value of time.

$$
\begin{aligned}
& x(t+\Delta t)=x(t)+v(t) \Delta t+\frac{1}{2} a(t) \Delta t^{2}, \\
& v(t+\Delta t)=v(t)+\frac{a(t)+a(t+\Delta t)}{2} \Delta t .
\end{aligned}
$$

In contrast, the leap-frog algorithm calculates the velocity and position of particles with a $\frac{1}{2} \Delta t$ difference.

\subsection{Observables and characterization}

To connect results from MD simulations to macroscopic theories, it is necessary to extract the material properties and macroscopic observables. Starting with positions, forces and velocities, it is possible to extract these from averages over space and time. These observables are calculated and stored for each time-step, both in order to monitor the equilibration of a system, and for its final analysis. Below we explain how to calculate some basic observables relevant to this thesis.

\subsubsection{Density, pressure and strain}

In MD simulations, the density $\rho$ is often expressed as a number density, describing the average number of particles present in certain volume $\left(\sigma^{-3}\right)$. The number density can be related to the volume fraction by multiplying the number of particles by the particle volume. Evaluating the density is necessary to distinguish the different phases present in a simulation. A density profile is shown in figure 2.4(b), corresponding to a simulation of a liquid-vapor interface (figure 2.4(c)). From figure 2.4(b) it is clear that on a nanoscale, a smooth interface region exists. The thermodynamic pressure $p$ can be computed using the virial expression [1]:

$$
p=\rho k_{\mathrm{B}} T-\frac{1}{d V_{\mathrm{sys}}}\left\langle\sum_{i<j} V^{\prime}\left(r_{i j}\right)\right\rangle,
$$


(a)

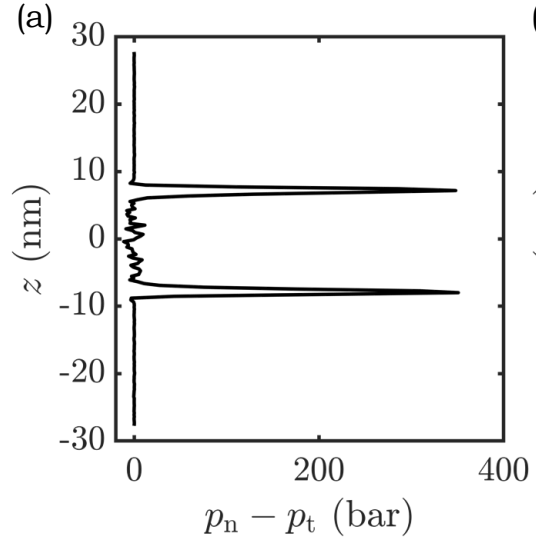

(b)

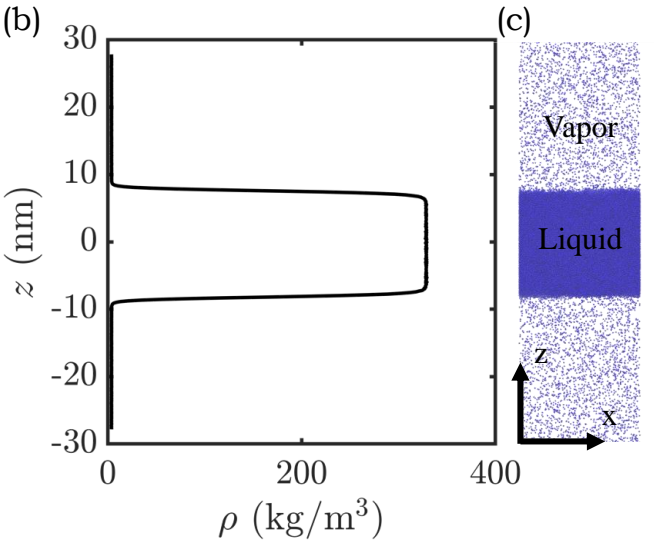

Figure 2.4: (a) A pressure profile and (b) density profile as a function of height for (c) a MD simulation of a Lennard-Jones liquid-vapor interface.

where $d$ is the dimensionality of the system and $V_{\text {sys }}$ is the system volume. The first part of this equation represents the contribution of the ideal gas law to the pressure, while the second accounts for the interactions. This equation expresses the pressure as a scalar value for the entire system. In our simulations however, we often calculate stress in different directions owing to anisotropy in the system. For example in figure 2.4, we have an interface in the $x y$-plane, meaning that one needs to define a normal pressure in the $z$-direction, and a tangential pressure in the $x$ and $y$ directions. Then we can define:

$$
\begin{gathered}
p_{\mathrm{N}}(z)=k_{\mathrm{B}} T\langle\rho(z)\rangle-\frac{1}{V_{\mathrm{sl}}}\left\langle\sum_{i \neq j} \frac{z_{i j}^{2}}{r_{i j}} V^{\prime}\left(r_{i j}\right)\right\rangle, \\
p_{\mathrm{T}}(z)=k_{\mathrm{B}} T\langle\rho(z)\rangle-\frac{1}{2 V_{\mathrm{sl}}}\left\langle\sum_{i \neq j} \frac{x_{i j}^{2}+y_{i j}^{2}}{r_{i j}} V^{\prime}\left(r_{i j}\right)\right\rangle,
\end{gathered}
$$

where $p_{\mathrm{N}}$ and $p_{\mathrm{T}}$ represent the pressure in the normal and tangential directions, $V_{\mathrm{sl}}$ is the sub-volume of a single slab.

In elastic media, we need to introduce the strain $\epsilon$. The strain is defined through a reference state. When a material is deformed by either a pulling or pushing force, the relative displacement is referred to as the strain. We remark that anisotropic pressures develop naturally in strained elastic media.

\subsubsection{Surface tension}

A clear illustration of the surface tension is given in figure 2.4(a), showing the stress anisotropy that is present at the interfacial region. The most often used 
method to determine this surface tension in MD simulations uses KirkwoodBuff's formula [1]:

$$
\Upsilon=\frac{1}{2} \int_{0}^{L_{z}}\left(p_{\mathrm{N}}(z)-p_{\mathrm{T}}(z)\right) d z,
$$

in which $L_{z}$ is the system size in direction $z$. In this equation, the factor $\frac{1}{2}$ accounts for the periodic boundaries, that allow for two surface tensions to exist. The pressures we use in equation 2.11 are gathered from equations 2.9 and 2.10 .

This description of the Kirkwood-Buff formula holds for simple liquid interfaces. In our case however, we also employ Kirkwood-Buff on more complex systems such as elastic gels and polymer brushes, where the definition of the interface location is less clear. The methods we use to analyse such materials will be discussed further in chapters 4 and 5 of this thesis.

\subsubsection{Contact angle}

In case of a liquid droplet wetting a surface in $\mathrm{MD}$, the wetting angle can be extracted from the data. Our fitting method is based upon the contact angle measurement method as described in [12. As the interface in MD is not a clearly defined line, but more an interface region, we start by fitting isodensity lines through a density map of the liquid droplet, as shown in figure 2.5(a). Figure 2.5(b) shows corresponding density profiles, and the width $w$ of the interface. Circles can be fitted through the isodensity contours, which describe the droplets' spherical cap shape. As the fitted circles all share a common center $C$, we can transform the circles to overlap all the fitted isodensity contours, and thus improving the statistics, as is shown in figure 2.5(c). The last step is to define the exact location of the interface of the droplet with its wetted surface, which is determined from the density profile for a bare substrate, to account for any deformation that might occur in soft substrates. The intersection between the fitted circle and the surface location, then provides the contact angle of the droplet.

\subsubsection{Overlap integrals and binary interactions}

As a tool to determine the state of a system (i.e. mixing or phase-separation), we use two methods that we refer to as overlap integrals, and binary interaction counts. The overlap integral calculates the proximity of two substances A and $\mathrm{B}$ to one another, by calculating the overlap integral of the phase densities $N_{\text {ov }}$. This overlap integral is defined as:

$$
N_{\mathrm{ov}}=\int_{0}^{\infty} \rho_{\mathrm{A}}(z) \rho_{\mathrm{B}}(z) d z,
$$




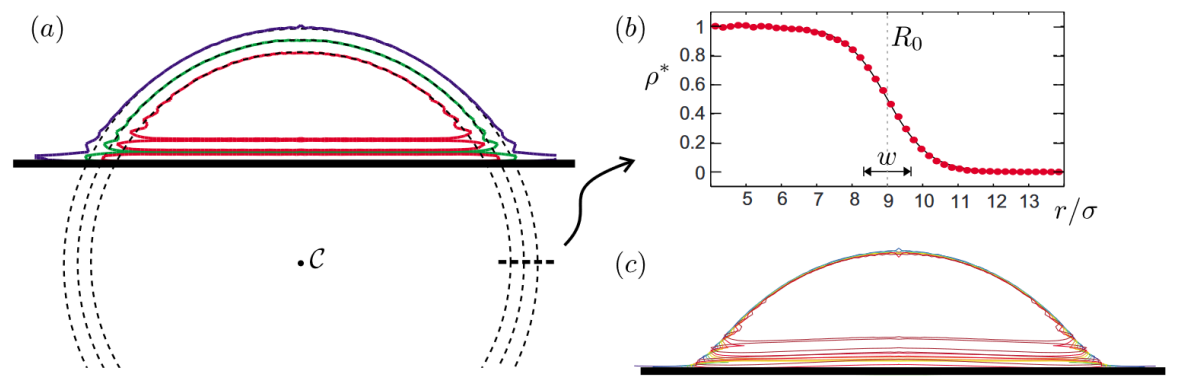

Figure 2.5: A schematic illustrating our contact angle-fitting protocol. (a) Isodensity contours of a LJ-droplet at relative densities $\rho^{*}=0.3,0.5,0.7$ (blue, green and red lines, respectively). The dashed lines show circles fitted through the isodensity contours. (b) The density profile showing the interface width $w$ of the droplet. (c) Rescaled isodensity contours collapse on a single curve. Image reproduced from Weijs et al. [12] with permission.

with $\rho_{\mathrm{A}}$ and $\rho_{\mathrm{B}}$ being the density profiles of both substances $\mathrm{A}$ and $\mathrm{B}$. A schematic illustrating such an overlap is shown in figure 2.6(a). In this graph, density profiles for two substances are shown. The red line shows the overlap of the two densities.

The binary interaction count is provides another tool to determine the state of the system. The binary interaction count, however, is more accurate when determining the system state in the case of an inhomogeneous distribution of the phases in a system, such as in local phase-separations. The binary interaction count $N_{\text {int }}$ identifies how many particles of a certain type are present around a set of reference particles (see also figure 2.6(b)). The binary interaction count is defined as:

$$
N_{\mathrm{int}}=\frac{1}{N_{\mathrm{RU}}} \sum_{\mathrm{i}=1}^{N_{\mathrm{RU}}} \sum_{\mathrm{j}=1}^{N_{\mathrm{MU}}} H\left(r_{c}-r_{i j}\right),
$$

where $N_{\mathrm{RU}}$ is the total number of reference particles in the simulation cell. $N_{\mathrm{MU}}$ is the total number of measured particles. $H\left(r_{i j}-r_{c}\right)$ is a Heaviside function which is 1 when the interparticle distance is smaller than the cut-off distance $r_{c}$, and 0 at larger distances.

\subsection{Set-up creation}

To create the initial configuration of a MD simulation, we take several steps and measurements in order to efficiently model polymer brushes and gels. In order to approach a large-scale macroscopic system in Molecular Dynamics, we make 
(a)

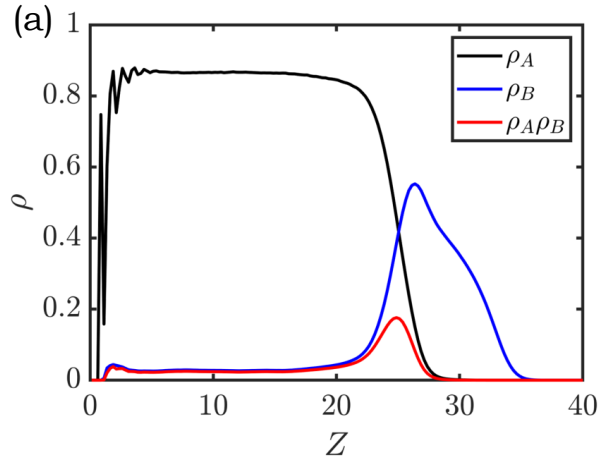

(b)

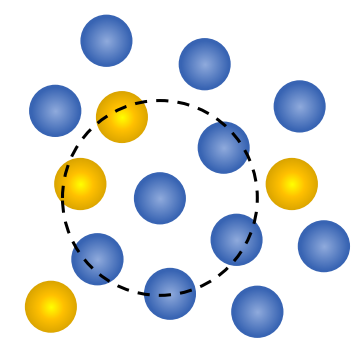

Figure 2.6: (a) Density profiles for a polymer brush and a polymer melt. The red line shows the overlap in the density profiles. (b) A schematic illustrating how the binary interactions are counted by taking one central particle, and looking within an interaction radius of that particle.

use of periodic boundaries. In such a case, particles that are positioned close to the boundary of the simulation box, will interact with the particles on the opposite side. As a consequence, particles are also capable of moving through the periodic boundaries, resulting in the particles reappearing on the other side of the periodic boundaries. As the number of particles in a MD simulation is limited due to the computational time needed, via periodic boundaries one is capable of simulating a bulk material.

Besides the periodic boundaries, we regularly choose our simulation box size as a so-called quasi-2D system. In such a system, one dimension is very small, creating a simplified version of a realistic system, saving expensive computational time. In the study of partially wetting systems, the quasi-2D approach may even be of advantage, as a phenomenon referred to as line tension has no effect on cilindrical droplets that would occur in a quasi-2D MD simulation [12.

Next, as we perform simulations on both gels and polymer brushes, it is important to be able to simulate these two complex materials correctly. Here we describe a short procedure we have used to create our polymer brushes and gels.

\subsubsection{Polymer brush}

The first step in our script is to read in the settings for the creation of the polymer brush. This includes parameters such as the brush polymer length $N$, polymer bead size $\sigma$, the interaction parameters $\epsilon$, the desired grafting density $\sigma_{\mathrm{GD}}$, the desired box dimensions $L_{x}, L_{y}, L_{z}$, and the radius of gyration of a single polymer chain of length $N$ in a bulk solution. 


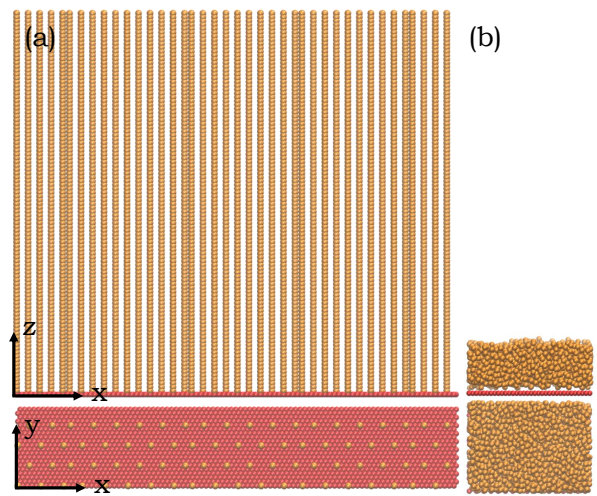

Figure 2.7: (a) A top and side view of the initial starting positions for a typical wall covered in polymer brushes. The wall is shown in red and the polymer brush in orange. (b) The same polymer brush after equilibration.

The second step is to calculate from these input parameters, the number of wall atoms that are needed, and the amount of polymer chains that are to be placed into the simulation box. The third step is to build the wall atoms into a Hexagonal Close Packed (HCP) unit cell structure. In step four the polymer chains are built, by placing consecutive beads on top of each other. An example of the initial starting positions of the polymer brush chains is shown in figure 2.7(a). After calculating the bead positions, the FENE bonds between consecutive beads are defined, as well as the FENE bond between the first bead and the wall atom. The last step in our script is to write out all the positions and bonds into an input file that is to be read by the MD simulator. After equilibration this brush will typically look like a brush as shown in figure 2.7(b).

\subsubsection{Polymer gel}

In order to create a polymer gel, we follow a procedure as in reference [13, 14. We start off with a polymer melt, that is created by placing a number of completely straightened polymer chains into a simulation box at random angles, as is depicted in figure 2.8(a). These polymer chains are then left to equilibrate and form a polymer melt. The next step is to then multiply this simulation box in the direction $x, y$ and $z$, to create the desired size dimensions for the resulting polymer gel. This new simulation box is then re-equilibrated to ensure a fully randomized polymer melt, as can be seen in figure 2.8(b).

From this polymer melt, we then create a polymer gel by crosslinking all the chains together to create a single gel molecule. This crosslinking is perhaps the most complex part of creating a polymer gel. First, we define the 
(a)

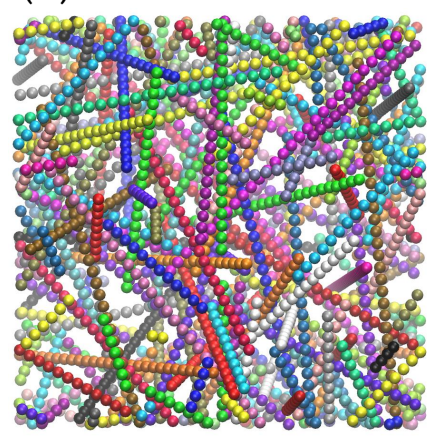

(b)

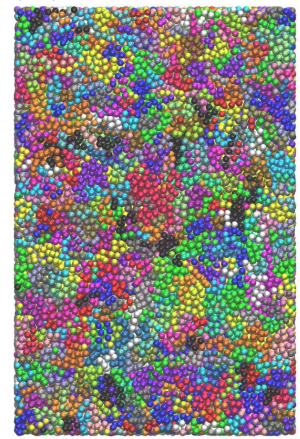

(c)

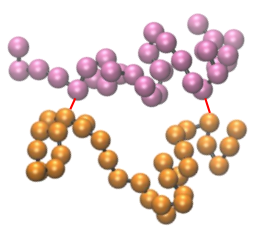

Figure 2.8: Visualisation of the gel-creating process. (a) The initial gel chains are placed into a simulation box at random angles and positions. (b) The polymer melt chains after equilibration. (c) Schematic of introducing crosslinks (red links) between two polymer chains.

input parameters, consisting of the positions and bonds of a polymer melt, a desired crosslinking density $n_{\mathrm{cr}}$, and the directions in which periodicity of the resulting gel is required. Next, a list of all possible crosslinks is created. The criteria for this list are that the selected particles are from two separate polymer chains, and that they are in close enough proximity to one another to be bonded through a FENE-bond (also see figure 2.8(c)). The possible crosslinks per chain are then sorted, according to the amount of crosslinks that are possible per chain. From the amount of possible crosslinks, we then calculate the probability with which each chain is to link to a neighbor. Next, we make an inventarisation of the end-beads of a chain, and their possibility to link to other chains. In order to keep the amount of crosslinks as homogeneous as possible, we use the following measures:

- We track all possible crosslinks for every polymer chain in the melt.

- We track the amount of crosslinks placed in subvolumes of the box.

- We make sure the amount of end-to-end crosslinks between polymer chains stays limited.

- We track the amount of crosslinks placed on every polymer chain.

- We make sure a single bead in the polymer chain cannot exceed 2 crosslinks.

- We make sure no loose chains remain present. 


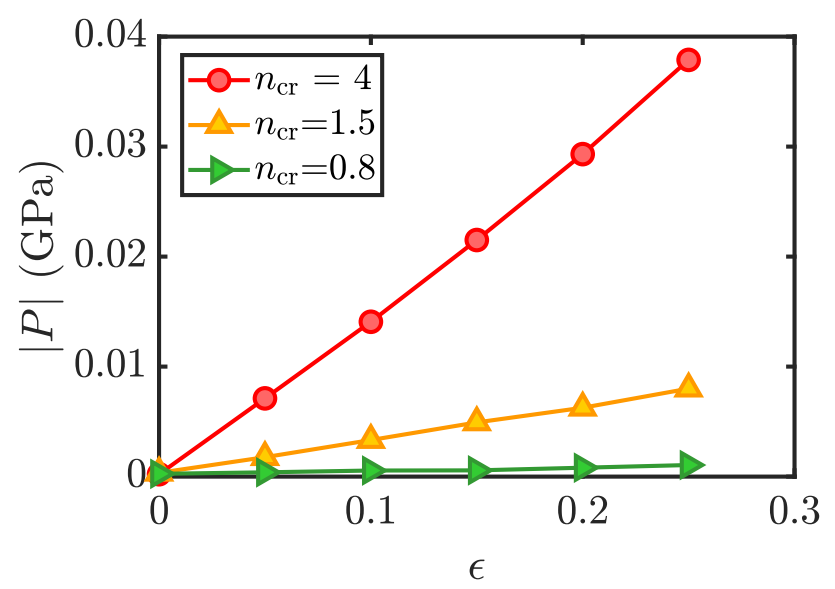

Figure 2.9: The stress-strain response of three gels with different crosslinking densities $n_{\text {cr }}$. Shown are 4 crosslinks per chain (red circles), 1.5 crosslinks per chain (yellow triangles) and 0.8 crosslinks per chain (green triangles), for an initial melt of $N=32$.

Using these conditions, we create a configuration containing the newly defined bonds, added to the pre-existing bonds and beads of the polymer melt. The rigidity of the resulting gel can be fine-tuned by increasing or decreasing the amount of crosslinks present. This influence of the crosslinking density on the gel stiffness is shown in figure 2.9, where the highest crosslinking density results in the largest elastic modulus.

\section{Bibliography}

[1] D. Frenkel and B. Smit, Understanding Molecular Simulation From Algorithms to Applications (Academic Press, San Diego, California) (1996).

[2] J. E. Jones and S. Chapman, "On the determination of molecular fields.-i. from the variation of the viscosity of a gas with temperature", Proceedings of the Royal Society of London. Series A, Containing Papers of a Mathematical and Physical Character 106, 441-462 (1924), URL https: //royalsocietypublishing.org/doi/abs/10.1098/rspa.1924.0081

[3] K. Kremer and G. S. Grest, "Dynamics of entangled linear polymer melts: A molecular-dynamics simulation", The Journal of Chemical Physics 92, 5057-5086 (1990), URL https://doi.org/10.1063/1.458541.

[4] G. S. Grest and K. Kremer, "Molecular dynamics simulation for polymers in the presence of a heat bath", Phys. Rev. A 33, 3628 (1986). 
[5] P. J. Flory, Principles of Polymer Chemistry (Cornell University Press, Ithaca) (1953).

[6] Y. Zhang, S. E. Feller, B. R. Brooks, and R. W. Pastor, "Computer simulation of liquid/liquid interfaces. i. theory and application to octane/water", The Journal of Chemical Physics 103, 10252-10266 (1995), URL https://doi .org/10.1063/1.469927.

[7] H. J. C. Berendsen, J. P. M. Postma, W. F. van Gunsteren, A. DiNola, and J. R. Haak, "Molecular dynamics with coupling to an external bath", The Journal of Chemical Physics 81, 3684-3690 (1984), URL https: //doi.org/10.1063/1.448118

[8] H. C. Andersen, "Molecular dynamics simulations at constant pressure and/or temperature", The Journal of Chemical Physics 72, 2384-2393 (1980), URL https://doi.org/10.1063/1.439486.

[9] W. G. Hoover, "Canonical dynamics: Equilibrium phase-space distributions", Phys. Rev. A 31, 1695-1697 (1985), URL https://link.aps .org/ doi/10.1103/PhysRevA.31.1695.

[10] M. Parrinello and A. Rahman, "Polymorphic transitions in single crystals: A new molecular dynamics method", Journal of Applied Physics 52, 71827190 (1981), URL https://doi.org/10.1063/1.328693.

[11] L. Verlet, "Computer "experiments" on classical fluids. i. thermodynamical properties of lennard-jones molecules", Phys. Rev. 159, 98-103 (1967), URL https://link .aps .org/doi/10.1103/PhysRev.159.98.

[12] J. H. Weijs, A. Marchand, B. Andreotti, D. Lohse, and J. H. Snoeijer, "Origin of line tension for a lennard-jones nanodroplet", Physics of Fluids 23, 022001 (2011), URL https://doi.org/10.1063/1.3546008

[13] H. Mehrabian, J. Harting, and J. H. Snoeijer, "Soft particles at a fluid interface", Soft Matter 12, 1062-1073 (2016), URL http://dx.doi.org/ 10.1039/C5SM01971K.

[14] H. Liang, Z. Cao, Z. Wang, and A. V. Dobrynin, "Surface stresses and a force balance at a contact line", Langmuir 34, 7497-7502 (2018), URL https://doi.org/10.1021/acs. langmuir.8b01680 


\section{Chapter 3}

\section{Wetting of Polymer Brushes by Polymeric Nanodroplets ${ }^{*}$}

End-anchoring polymers to a solid surface to form so-called polymer brushes is a versatile method to prepare robust functional coatings. We show, using molecular dynamics simulations, that these coatings display rich wetting behavior. Depending on the interaction between the brushes and the polymeric droplets as well as on the self-affinity of the brush, we can distinguish between three wetting states: mixing, complete wetting and partial wetting. We find that transitions between these states are largely captured by enthalpic arguments, while deviations to these can be attributed to the negative excess interfacial entropy for the brush droplet system. Interestingly, we observe that the contact angle strongly increases when the softness of the brush is increased, which is opposite to the case of drops on soft elastomers. Hence, the Young to Neumann transition owing to softness is not universal, but depends on the nature of the substrate.

\subsection{Introduction}

Soft brush-like structures are found in multiple places in nature, for example in human joints, intestines and lungs, where they aid in tasks such as lubrication, filtering, absorption and antifouling [1-3]. In a biomimetic approach, most of these functionalities can be obtained by grafting polymers at a high density to a surface to form so-called polymer brushes [4-7]. Research interest in these polymer brushes has grown rapidly in recent years due to its potential for applications, e.g. as smart adhesives [8 10], as sensors [11-13], in gating [14-

${ }^{*}$ Published as: L.I.S. Mensink, J. H. Snoeijer, and S. de Beer, Wetting of Polymer Brushes by Polymeric Nanodroplets, Macromolecules 52, 5, 2015-2020 (2019). 
16], in moisture management systems [17, 18] and on self-cleaning surfaces [19, 20]. For many of these applications, it is important to understand how droplets interact with soft brush structures.

Recently, wetting of droplets on soft substrates has gained a lot of attention [21 35]. Wetting on these substrates can be very different from that on rigid substrates, because surface tension can deform the substrates [30, 31, 33. Considering the rigidity of the substrates, wetting behavior can be categorized in three regimes depending on the elasto-capillary length, which is defined as the surface free energy $\gamma$ divided by Young's modulus $E$. When $\gamma / E$ is much smaller than the range of molecular interactions $a$, surfaces are not deformed and Young's law applies. When $\gamma / E$ is larger than $a$, wetting ridges are formed [21, 23, 34], which alter the microscopic contact angle, yet do not affect the macroscopic contact angle $\theta$. The macroscopic contact angle will deviate from predictions by Young's law only when $\gamma / E$ is comparable to the droplet-size $R$. For larger $\gamma / E, \theta$ becomes increasingly smaller and approaches Neumann's law in the limit of $\gamma / E \gg R[25,27]$.

For substrates composed of polymer brushes, one can anticipate even richer wetting behavior. The reason for this is that end-anchoring of the polymers imposes translational constraints that allow for wetting by liquids that would otherwise dissolve the polymers and thereby degrade the coating [36]. Moreover, the reduction of configurational entropy for surface-attached polymers can give rise to counter-intuitive effects such as autophobic dewetting of chemically identical polymer films $[37-40]$. Previous work revealed the formation of wetting ridges for droplets on brushes [41. However, so far, a complete overview of how brush softness in combination with brush-droplet affinity affects the wetting of brushes is still lacking.

In this article, we explore the wetting behavior of polymer brushes by polymer droplets, under a wide variety of conditions. Using molecular dynamics simulations, we reveal three wetting states: mixing, complete wetting and partial wetting, which can be controlled by the interactions between the brush polymers and the droplet relative to the interaction between the polymers in the brush. In the partial wetting state, we observe various phenomena that depend on the softness of the brush. Interestingly, we do not observe $\theta$ to decrease with increasing softness of the brush as observed for elastomers. Instead, we observe the opposite trend and that $\theta \rightarrow 180^{\circ}$ for soft brushes.

\subsection{Model and Methods}

The polymers are represented by a coarse-grained bead-spring model (KremerGrest model [42]), which is known to capture the generic traits of bulk poly- 
mers [43, polymers in solvent(-mixtures) 44] as well as polymer brushes [45, 46]. Within our Kremer-Grest based model, the non-bonded interactions within and between brush and liquid are also described by a Lennard Jones potential:

$$
V_{\mathrm{LJ}}=4 \epsilon\left(\left(\frac{\sigma}{r}\right)^{12}-\left(\frac{\sigma}{r}\right)^{6}\right),
$$

using $\sigma=1$ and $r_{\mathrm{c}}=2.5 \sigma$ [47]. Within the Lennard Jones potential, $\sigma$ is the radius where the potential is zero and a representation for the size of the polymer bead. The parameter $\epsilon$ equals the potential well depth and is our unit of energy. The Lennard-Jones units can be translated to real values for polymers such as poly(ethylene) using $\epsilon=30 \mathrm{meV}$ and $\sigma=0.5 \mathrm{~nm}$ [43]. Consecutive beads interact via the finite extensible nonlinear elastic (FENE) bond (spring stiffness $k=30 \epsilon / \sigma^{2}$ and maximum extent $R_{0}=1.5 \sigma$ ), while overlap of the beads is inhibited by a Lennard Jones potential that is cut off in the potential minimum (interaction strength $\epsilon=1$, zero-crossing distance for the potential $\sigma=1$, cut-off radius $\left.r_{\mathrm{c}}=2^{1 / 6} \sigma\right)$. A polymer bead represents typically 3-4 monomers. Therefore, the unit of mass $[\mathrm{m}]$ is $10^{-22} \mathrm{~kg}$ and the unit of time $[\tau]$ represents $0.3 \mathrm{~ns}[43$.

The configurations shown in figure 3.1 (a-c) are extracted snapshots of our simulation cells [48]. The simulations are performed at a constant box-size (constant volume $V$ ) in a quasi-2D setup to prevent line-tension effects [49]. Boundary conditions are periodic in $x$ and $y$ and the boxlength is limited to $15 \sigma$ in $y$ to suppress the Rayleigh instability in the infinitely long cylindrical droplet (figure 3.1(c)). All simulation cells contain surfaces with high density polymer brushes attached to them (orange, figure 3.1). The grafting density is 0.15 chains per unit area, which is $20 \mathrm{x}$ the critical grafting density for brush formation [50]. This density is in the high density regime [51] as is commonly obtained in laboratories using the 'grafting from' method [8, 52]. Each brush-polymer consists of $N_{\mathrm{B}}=100$ repeat units and is allowed to interact with a droplet containing 485 polymers, each of $N_{\mathrm{L}}=32$ repeat-units (blue, figure 3.1.

The equations of motion are solved using the Verlet algorithm as implemented in LAMMPS [53] using a timestep of $\Delta t=0.005 \tau$. The simulations are performed in the $N V T$ ensemble and the temperature $T$ is kept constant at $k_{\mathrm{B}} T=1 \epsilon\left(k_{\mathrm{B}}\right.$ being the Boltzmann constant) using a Langevin thermostat (damping-coefficient $\xi=1 \tau^{-1}$ ). We vary $\epsilon_{\mathrm{BB}}$ between 0.5 and 2 . By varying $\epsilon_{\mathrm{BB}}$, we vary implicitly the interaction of the brush with the implicit solvent. When $\epsilon_{\mathrm{BB}}$ is high, the brush-polymers like themselves and, thereby, dislike the implicit solvent. In contrast, when $\epsilon_{\mathrm{BB}}$ is low, the self-interaction within the 
brush-polymers is low and the polymers prefer the implicit solvent.The variation in $\epsilon_{\mathrm{BB}}$ can be related to effective self-interaction parameters $\tau=\epsilon_{\mathrm{BB}} / \epsilon_{\mathrm{BB}, \theta}$ between 1.6 and 6.5. In this equation $\epsilon_{\mathrm{BB}, \theta}=0.31$ is the $\theta$-transition point below which the brush is in implicit good solvent conditions. We employ a generic LJ interaction and do not intend to model particular types of polymers. Moreover, we do not limit ourselves to systems described by van der Waals interactions alone. Therefore, mixing rules are not strict [54 and we can alter $\epsilon_{\mathrm{BB}}$ and $\epsilon_{\mathrm{BL}}$ independently. This will make our results broadly applicable. We vary $\epsilon_{\mathrm{BL}}$ between 0.125 and 1.75 , while $\epsilon_{\mathrm{LL}}=1$ is kept constant. In experiments these interactions can be altered by choosing different combinations of polymers. The interactions between the wall and the polymer- or liquid-beads is purely repulsive $\left(\epsilon=1, \sigma=1\right.$ and $\left.r_{\mathrm{c}}=2^{1 / 6} \sigma\right)$ to prevent preferential adsorption near the wall [55]. Due to our choice for the wall-interactions as well as the thickness of our polymer film, there will also be no wall-induced wetting transitions [56].

\subsection{Results and Discussion}

The phase diagram of figure 3.1(d) depicts how the wetting regimes depend on the affinity of the brush with the droplet ( $x$-axis) as well as the self-interaction of the brush $\epsilon_{\mathrm{BB}}$ ( $y$-axis). The brush-droplet affinity is characterized by the interaction parameter $W_{\mathrm{BL}}$, which we define as $W_{\mathrm{BL}}=\frac{1}{2}\left(\epsilon_{\mathrm{BB}}+\epsilon_{\mathrm{LL}}\right)-\epsilon_{\mathrm{BL}}$. It gives the droplet-brush affinity relative to the self-interactions within the droplet and the brush. Our $W_{\mathrm{BL}}$ can be related to the traditional Flory Huggins parameter [57, for more information on this translation we refer to Ref 58 and 59. The swelling of the brushes is controlled by $\epsilon_{\mathrm{BB}}$. A large $\epsilon_{\mathrm{BB}}$ models a hard, rigid brush, while a small $\epsilon_{\mathrm{BB}}$ results in a softer brush.

We first focus on the red region of the phase diagram in figure 3.1(d), where the interactions are such that deposited droplets mix with the brush polymers. Depending on $\epsilon_{\mathrm{BB}}$ different melt partitioning regimes can be identified. We observe that the composition of the brush-surface varies between melt-enriched for large $\epsilon_{\mathrm{BB}}$ (see figure 3.2 (a) for $\epsilon_{\mathrm{BB}}=2 \epsilon_{\mathrm{LL}}$ ) to brush-enriched for small $\epsilon_{\mathrm{BB}}$ (see figure 3.2 (b) for $\epsilon_{\mathrm{BB}}=0.5 \epsilon_{\mathrm{LL}}$ ). The latter regime has also been predicted by self consistent field theory calculations [60]. The reason for such a non-uniform distribution and variation in interfacial composition is that the medium with the lower self-affinity will pay a smaller energy penalty for residing at the interface.

Upon increasing $W_{\mathrm{BL}}$, we observe a transition from mixing to partial wetting for small $\epsilon_{\mathrm{BB}}$ and to complete wetting for large $\epsilon_{\mathrm{BB}}$. To identify the exact transition- $W_{\mathrm{BL}}\left(W_{\mathrm{BL}, \mathrm{TR}}\right)$, we calculate the binary interaction count $N_{\text {int }}$ (see 


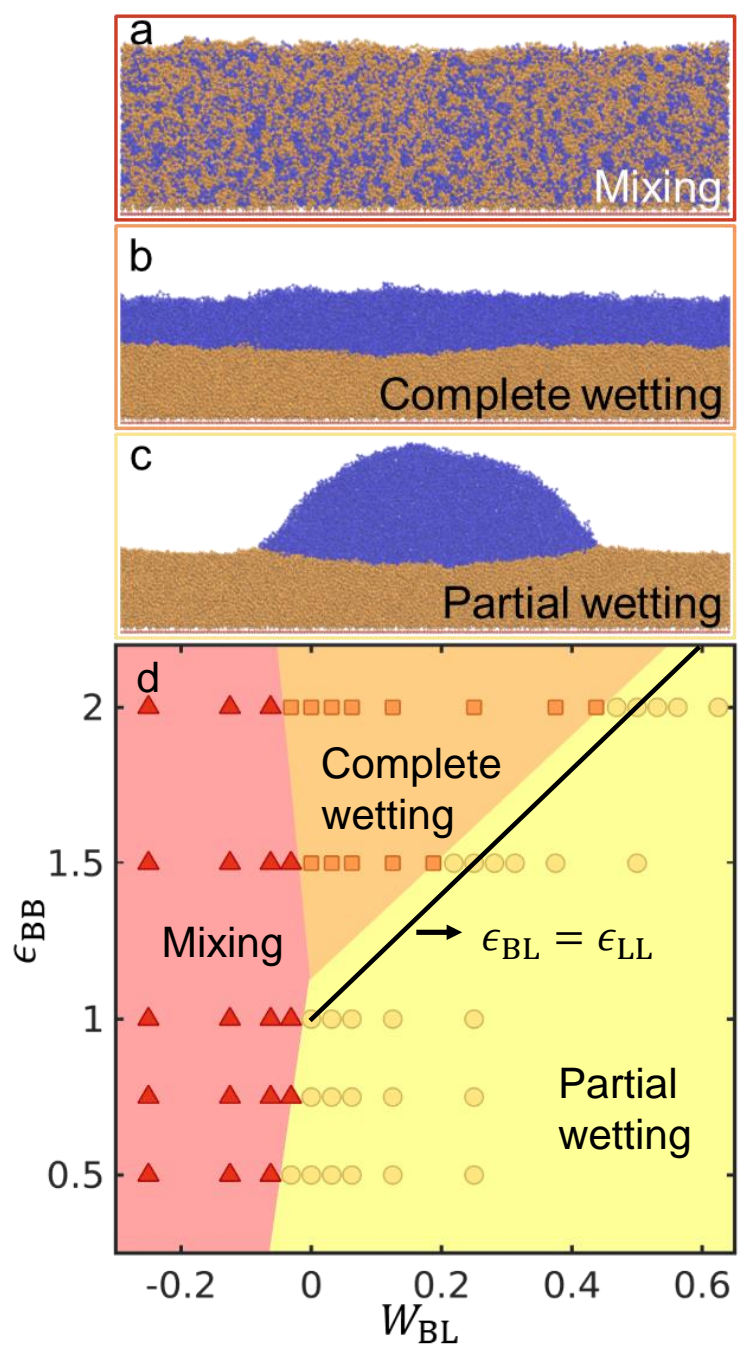

Figure 3.1: (a-c) Snapshots of simulation cells showing a polymer droplet (blue), interacting with a polymer brush (orange) for the three wetting states. (d) Phase diagram depicting the relation between the states of wetting and brush self-interaction $\epsilon_{\mathrm{BB}}$ and the interaction parameter $W_{\mathrm{BL}}$. Observed are mixing (red triangles), complete wetting (orange squares) and partial wetting states (yellow circles). The black line indicates the enthalpic prediction for the transition from complete to partial wetting $\left(\epsilon_{\mathrm{BL}}=\epsilon_{\mathrm{LL}}\right)$. 
appendix 3.5. figure 3.4 61], which is high for mixing systems and low for phase-separated systems. We define $N_{\text {int }}$ as

$$
N_{\mathrm{int}}=\frac{1}{N_{\mathrm{BU}}} \sum_{i=1}^{N_{\mathrm{BU}}} \sum_{j=1}^{N_{\mathrm{LU}}} H\left(r_{i j}-r_{\mathrm{c}}\right),
$$

where $N_{\mathrm{BU}}$ is the total number of brush units in the simulation cell, $N_{\mathrm{LU}}$ is the total number of liquid units and $H\left(r_{i j}-r_{\mathrm{c}}\right)$ is a Heaviside function, which is 1 when the interparticle distance $r_{i j}$ is smaller than $r_{\mathrm{c}}=1.5 \sigma$.

Figure 3.2(c) shows $N_{\text {int }}$ for various $\epsilon_{\mathrm{BB}}$ between 0.5 and 2. We define $W_{\mathrm{BL}, \mathrm{TR}}$ as the halfway point of the hyperbolic tangent fitted to the data. The $W_{\mathrm{BL}, \mathrm{TR}}$ is negative for all $\epsilon_{\mathrm{BB}}$ (see inset figure 3.2(c)). A negative $W_{\mathrm{BL}, \mathrm{TR}}$ might seem counter-intuitive, because this implies that mixing reduces the entropy of the system. However, it can be understood using similar arguments as for autophobic dewetting [37]. The reason for the observed effect is that end-anchored polymers are constrained and, therefore, they do not gain translational entropy upon mixing. Instead, they pay an entropic penalty for stretching when absorbing the polymer melt. If the polymers in the melt are sufficiently long, their gain in translational entropy upon absorption in the brush is too small to overcome the reduction in entropy due to stretching of the polymers of the brush such that the system will not mix. This is consistent with previous studies on mixing / demixing of brushes with chemically identical melts [62, 63], which suggest a demixed state at $W_{\mathrm{BL}}=0$ for our grafting density and $N_{\mathrm{L}} / N_{\mathrm{B}}=0.35$. Therefore, our mixing-demixing transitions should occur at negative $W_{\mathrm{BL}}$.

In contrast to predictions [64, the observed $W_{\mathrm{BL}, \mathrm{TR}}$ is not constant. Instead, it increases with increasing $\epsilon_{\mathrm{BB}}$ for $\epsilon_{\mathrm{BB}}<0.75 \epsilon_{\mathrm{LL}}$, while it decreases with increasing $\epsilon_{\mathrm{BB}}$ for $\epsilon_{\mathrm{BB}}>1 \epsilon_{\mathrm{LL}}$ (see inset figure $3.2(\mathrm{c})$ ). This demonstrates that Flory-Huggins or scaling theories cannot be directly applied to our system. The reason for this is that the volume conservation and the incompressibilityassumptions are invalid due to the compressibility of the implicit solvent. Indeed, inspection of the average densities of the liquid and the brush reveals that mixing alters the average free volume. Similar conditions apply in the lab, where the droplet and brush are in equilibrium with (compressible) air.

Now we turn to the right side of the phase diagram of figure 3.1(d), where the melt and the brush do not mix. In the orange region of the phase diagram, the liquid completely wets the brush, while in the yellow region, the melt partially wets the brush and takes the shape of a droplet. It is possible to link the transition between partial and complete wetting for our brush system to the well-known wetting transition for non-absorbing surfaces described by the Young-Dupré law. For this, we relate the spreading parameter $S$ defined 

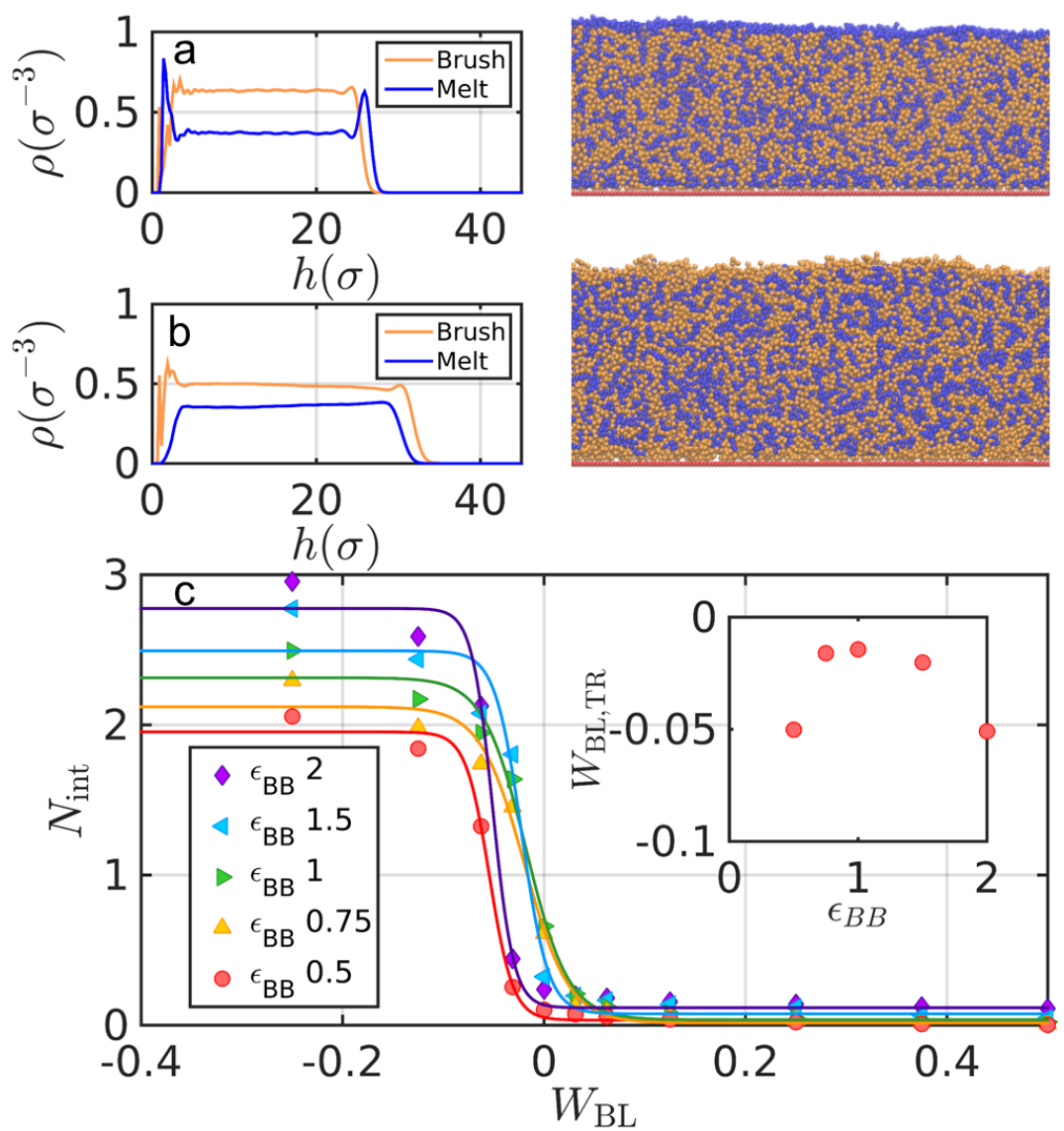

Figure 3.2: (a-b) Density profiles of a polymer melt (blue) mixed into a polymer brush (orange), (a) shows mixing in a collapsed brush $\left(\epsilon_{\mathrm{BB}}=2 \epsilon_{\mathrm{LL}}\right)$, (b) shows mixing in an initially slightly extended brush $\left(\epsilon_{\mathrm{BB}}=0.5 \epsilon_{\mathrm{LL}}\right)$. (c) Binary interaction count for different polymer brushes $\left(\epsilon_{\mathrm{BB}}\right.$, given in the legend) interacting with a polymer liquid, for different interaction parameters $W_{\mathrm{BL}}$. The inset shows the transition $W_{\mathrm{BL}}$. 
by the solid-liquid, solid-vapor and liquid-vapor surface tensions, $\gamma_{\mathrm{SL}}, \gamma_{\mathrm{SV}}$ and $\gamma_{\mathrm{LV}}$, respectively, to the interaction parameter $\epsilon$. This relation can be found considering the work of adhesion upon separation, which is proportional to the strength of the interaction $\epsilon$ between the media before separation. [54] Upon separating two halfspace media, two new interfaces between the media and the air (vapor) are created, each of which has a surface energy $\gamma$. If the two media consist of the same liquid, the work of adhesion $W=2 \gamma_{\mathrm{LV}}$ and this must be proportional to $\epsilon_{\mathrm{LL}}$. Similarly, for the same (brush) solids $W=2 \gamma_{\mathrm{SV}}$ and this expression scales with $\epsilon_{\mathrm{BB}}$. If a (brush) solid and a liquid are separated, we need to correct for the initial interfacial energy $\gamma_{\mathrm{SL}}$ such that $W=\gamma_{\mathrm{SV}}+\gamma_{\mathrm{LV}}-\gamma_{\mathrm{SL}}$ and this should scale with $\epsilon_{\mathrm{BL}}$. By these enthalpic considerations, the spreading parameter becomes $S=\gamma_{\mathrm{SV}}-\left(\gamma_{\mathrm{SL}}+\gamma_{\mathrm{LV}}\right) \propto \epsilon_{\mathrm{BL}}-\epsilon_{\mathrm{LL}}$. When $S<0$ the liquids partially wets the surface, while for $S>0$ complete wetting occurs. Therefore, the partial to complete wetting transition is expected to occur at $\epsilon_{\mathrm{BL}}=\epsilon_{\mathrm{LL}}$, if enthalpic interactions determine the transition. Moreover, using Young's law for $S<0$, we can write $S=\gamma_{\mathrm{LV}}(\cos \theta-1)$, which leads to the estimation for the contact angle as $\cos \theta=2 \epsilon_{\mathrm{BL}} / \epsilon_{\mathrm{LL}}-1$.

Comparing the model predicting the partial to complete wetting transition $\left(\epsilon_{\mathrm{BL}}=\epsilon_{\mathrm{LL}}\right.$, black line figure 3.1(d)) with the simulation results (orange and yellow in figure 3.1(d), see supporting information for typical snapshots near the transition), we find a reasonable qualitative agreement. However, it is clear that this transition is shifted to smaller $W_{\mathrm{BL}}$ for all $\epsilon_{\mathrm{BB}}$. The reason for this shift has the same roots as the shift in $W_{\mathrm{BL}, \mathrm{TR}}$. Namely, it can be attributed to the entropic penalty that our setup pays for mixing [37, 62]. The entropic penalty, or negative excess entropy, increases the effective interfacial free energie between the brush and the liquid $\gamma_{\mathrm{SL}}$ [65, 66]. This increase in $\gamma_{\mathrm{SL}}$ reduces the spreading parameter $S$ such that partial wetting is observed for $\epsilon_{\mathrm{BL}}=\epsilon_{\mathrm{LL}}$. The latter has been confirmed with experiments of autophobic dewetting, where indeed finite contact angles are observed for melt droplets on the chemically identical high-density brushes [39, 67, 68]. This shows that our setup models experimental systems reasonably well, despite our system size being smaller. Moreover, preliminary tests show that the transition-shift and, thus, the entropic contribution to the interfacial free energy strongly depends on the degree of polymerization of the droplet-polymers $N_{\mathrm{L}}$, in agreement with experimental observations [39].

To further examine the partial wetting state, we determine the contact angles of the droplets on the brushes (see figure 3.3). The contact angle is extracted by spherical fits to the top part of the droplet that rises above the height of the unperturbed brush. Figure 3.3 shows the contact angles $\theta$ for brush-droplet combinations in the partial wetting regime. We plot $\theta$ extracted 


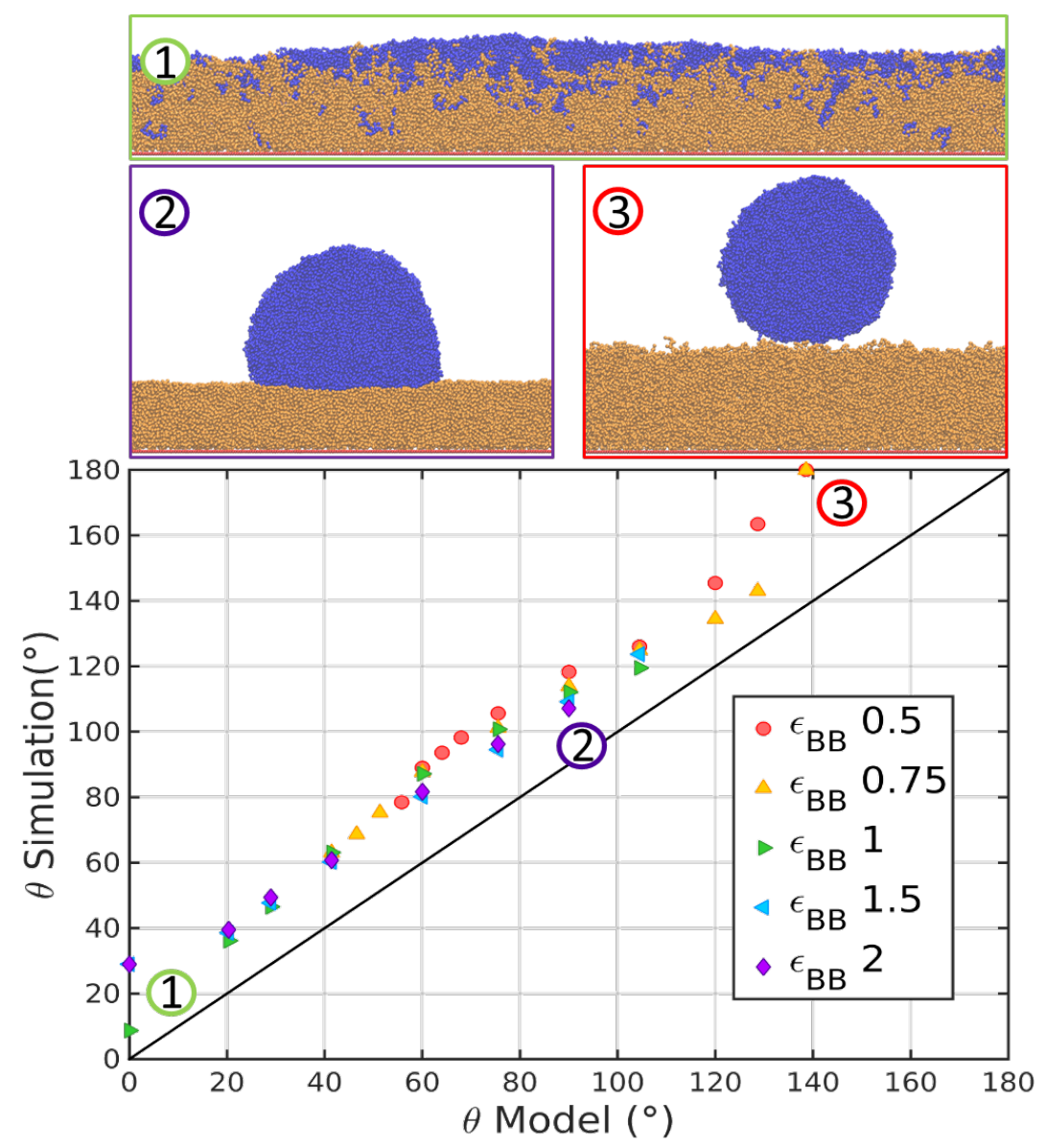

Figure 3.3: Snapshots showing the typical partial wetting states with the corresponding numbering referring to their data points in the graph. Below is a plot of the contact angles extracted from the simulations versus the contact angles predicted from enthalpic arguments. The individual series are at constant $\epsilon_{\mathrm{BB}}$ (given in the legend), and are all varied in $\epsilon_{\mathrm{BL}}$. 
from the simulations as a function of the contact angle predicted from the enthalpic model for Young's law $\cos \theta=2 \epsilon_{\mathrm{BL}} / \epsilon_{\mathrm{LL}}-1$. The contact angles extracted from the simulations are always more than $20^{\circ}$ higher than expected from enthalpic interactions. This increase in the contact angle can also largely be explained by the entropic penalty-induced increase in $\gamma_{\mathrm{SL}}$, [65, 66] which will effectively increase the contact angle.

For the snapshots in figure 3.3, we can distinguish between three partial wetting regimes depending on $\epsilon_{\mathrm{BB}}$ and $\epsilon_{\mathrm{BL}}$. For $\epsilon_{\mathrm{BB}}>1$, the brush surface is not deformed by the surface tension of the droplet (see figure 3.3, snapshot 2). In this regime, Young's law should be valid when entropic contributions are taken into account. For $\epsilon_{\mathrm{BB}} \leq 1$, the brush is soft enough such that wetting ridges are formed (see appendix 3.5, figure 3.5), consistent with observations by Léonforte et al. [41] The height of the wetting ridges slightly increases from $4 \sigma$ to $5 \sigma$ upon decreasing $\epsilon_{\mathrm{BB}}$ from 1 to 0.5 (see appendix 3.5, figure 3.5), because a reduction in $\epsilon_{\mathrm{BB}}$ decreases the stiffness of the brush. The height of the ridges also increases (approximately 300\%) with increasing $\epsilon_{\mathrm{BL}}$ from 0.5 to 0.75 , because this reduces $\gamma_{\mathrm{SL}}$ counteracting the deformation.

In contrast to reports on droplets on soft gels [25, 26], we observe no Young to Neumann transition and the according decrease in contact angles for soft brushes and high $\gamma_{\mathrm{SL}}$. Instead, we observe an increase in the contact angle to even $180^{\circ}$ for small $\epsilon_{\mathrm{BB}}$ and $\epsilon_{\mathrm{BL}}$ (see figure 3.3, snapshot 3). This is surprising, because the effect cannot be attributed to the deformability of the substrate and should, therefore be caused by the interactions. However, $\epsilon_{\mathrm{BB}}$ does not affect Young's law estimated from enthalpic arguments. To understand our observed trend in $\theta$, we have to consider that we have three components in our system and that the brush-melt interactions are mediated by the implicit solvent. By reducing $\epsilon_{\mathrm{BB}}$, we increase the affinity of the brush with the implicit solvent, such that we effectively reduce the affinity of the brush with the polymer droplet. This increases the contact angle. Moreover, at low $\epsilon_{\mathrm{BB}}$, the density of the brush decays with the distance from the surface, such that the droplet's inclusion free energy, which strongly increases with the density of the brush [46], is the lowest in the top of the brush. Therefore, the melt is expelled to the top of the brush. Our results imply that the Young to Neumann transition is not universal. However, the results do not exclude that such a transition could still occur for softer brushes of lower grafting densities.

\subsection{Conclusions}

In summary, we have shown that there are three wetting states for polymeric nanodroplets in contact with brushes: mixing, complete wetting and 
partial wetting. The transitions between mixing and demixing and partial and complete wetting are largely determined by enthalpic interactions. However, detailed examination reveals significant entropy-induced deviations. The transition- $W_{\mathrm{BL}}$ for mixing is always slightly negative, independent of the selfaffinity of the brush $\epsilon_{\mathrm{BB}}$. This implies that there is a decrease in entropy upon mixing for our choice of system parameters (polymer length and grafting density). This effect is caused by the entropic penalty for stretching of the brush-polymers upon mixing, which is not sufficiently compensated for by the gain in translational entropy of the melt-polymers. Above the transition- $W_{\mathrm{BL}}$, we observe complete wetting for large $\epsilon_{\mathrm{BB}}$ and partial wetting for small $\epsilon_{\mathrm{BB}}$. Also the transition from partial to complete wetting is shifted to smaller $W_{\mathrm{BL}}$ than expected from the Young-Dupré equation considering only enthalpic interactions. The reason for this is that the negative excess interfacial entropy between the brush and the droplet effectively increases the interfacial free energy $\gamma_{\mathrm{SL}}$ between them. As a consequence, the contact angles extracted from the simulations in the partial wetting regime are also consistently higher than expected from Young's law considering only enthalpic interactions. Interestingly, we find that in the limit of high $\gamma_{\mathrm{SL}}$ and low brush stiffness, contact angles increase and approach $180^{\circ}$. This is the opposite of what is generally reported for droplets in contact with soft gels, where contact angles are observed to decrease under these conditions. This reveals that the Young to Neumann transition owing to softness cannot be considered as universal: It depends on the specific nature of the substrate. These observations will impact the design and functionality of soft surfaces in terms of wetting and adhesive performance. 


\subsection{Appendix}

\subsubsection{Transition regime}
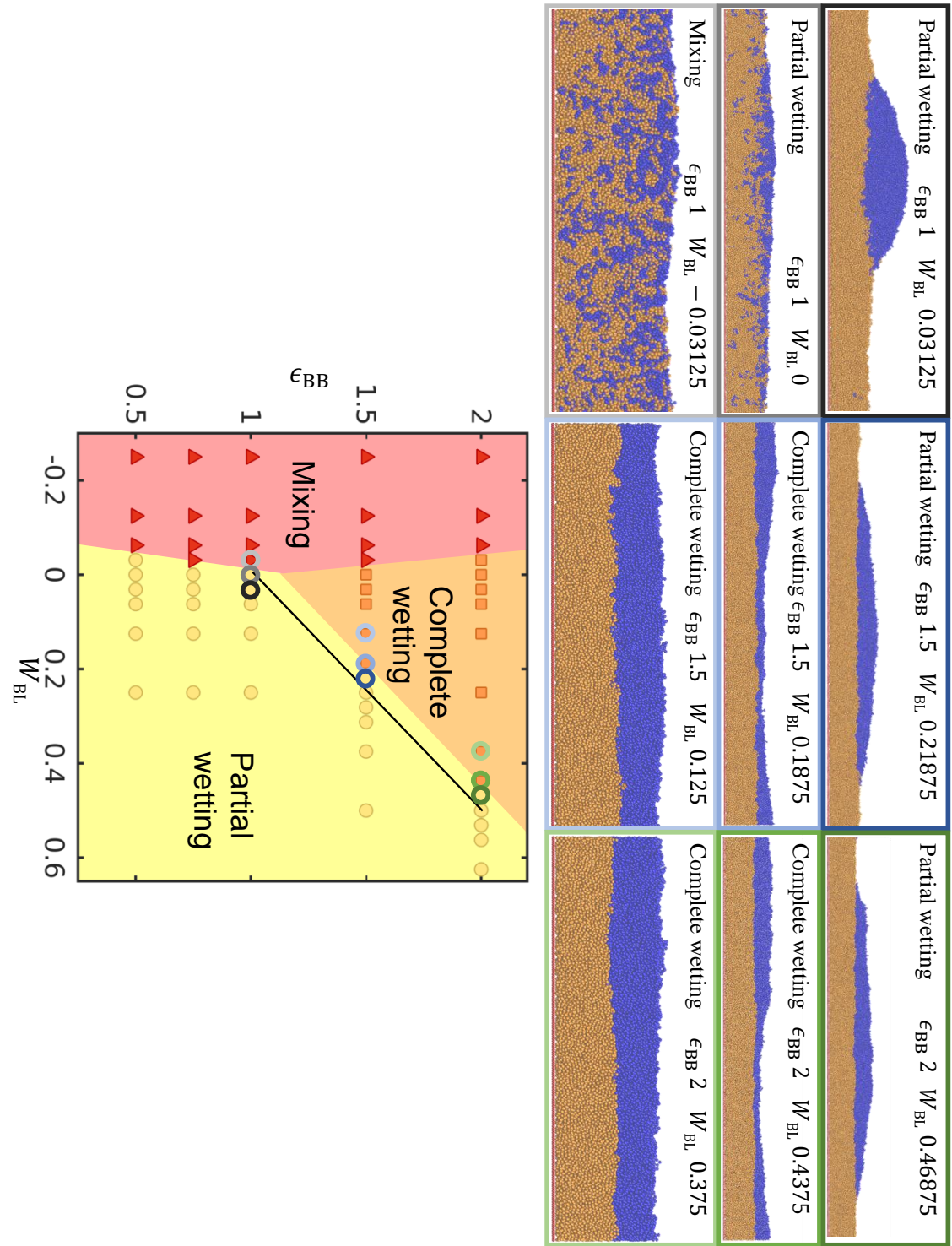

Figure 3.4: A table showing snapshots of the brush-droplet system for settings near the transition points. The recorded state and settings are shown above the corresponding snapshots. 


\subsubsection{Wetting ridges}

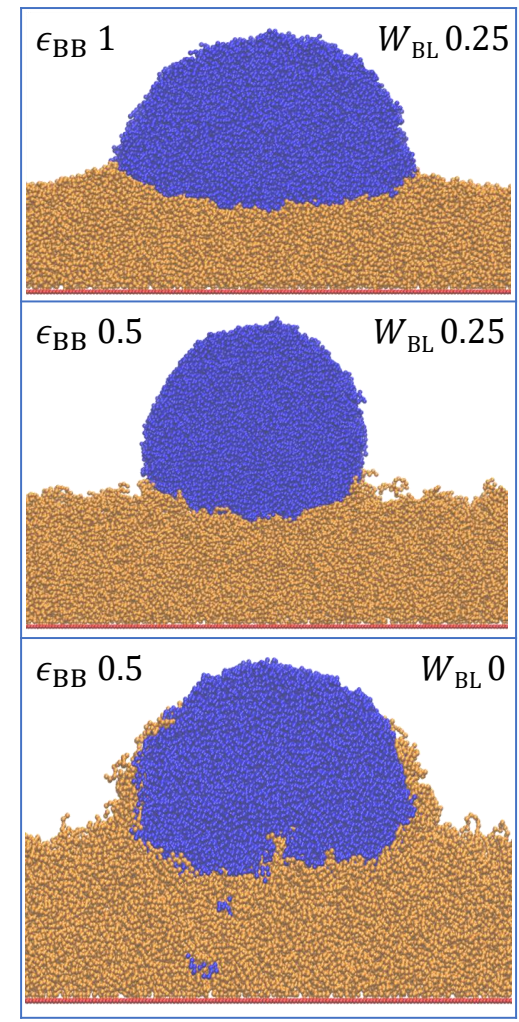

Figure 3.5: A table showing snapshots of the brush-droplet system, showing wetting ridges formed by the polymer brushes at different degrees of collapsed brush and brush wettability.

\section{Bibliography}

[1] J. Klein, "Repair or replacement-a joint perspective", Science 323, 47-48 (2009), URL http://science.sciencemag.org/content/323/5910/47.

[2] M. E. V. Johansson, D. Ambort, T. Pelaseyed, A. Schütte, J. K. Gustafsson, A. Ermund, D. B. Subramani, J. M. Holmén-Larsson, K. A. Thomsson, J. H. Bergström, S. Van Der Post, A. M. Rodriguez-Piñeiro, H. Sjövall, M. Bäckström, and G. C. Hansson, "Composition and functional role of the mucus layers in the intestine", Cellular and Molecular Life Sciences 68, 3635-3641 (2011). 
[3] B. Button, L.-H. Cai, C. Ehre, M. Kesimer, D. B. Hill, J. K. Sheehan, R. C. Boucher, and M. Rubinstein, "A periciliary brush promotes the lung health by separating the mucus layer from airway epithelia", Science 337, 937-941 (2012), URL http://science.sciencemag.org/content/337/ 6097/937.

[4] A. C. Balazs, T. Emrick, and T. P. Russell, "Nanoparticle polymer composites: Where two small worlds meet", Science 314, 1107-1110 (2006), URL http://science.sciencemag. org/content/314/5802/1107.

[5] M. Chen, W. H. Briscoe, S. P. Armes, and J. Klein, "Lubrication at physiological pressures by polyzwitterionic brushes.", Science 323, 1698-1701 (2009).

[6] H. Angus, R. Srinath, and C. Ashutosh, "In pursuit of zero: Polymer brushes that resist the adsorption of proteins", Advanced Materials 21, 2441-2446 (2009), URL https://onlinelibrary.wiley.com/doi/abs/ 10.1002/adma. 200900383 .

[7] S. de Beer, E. Kutnyanszky, P. M. Schön, G. J. Vancso, and M. H. Müser, "Solvent-induced immiscibility of polymer brushes eliminates dissipation channels.", Nature communications 5, 3781 (2014), URL http://www. ncbi.nlm.nih.gov/pubmed/24828542.

[8] Y. Yu, B. D. Kieviet, E. Kutnyanszky, G. J. Vancso, and S. de Beer, "Cosolvency-induced switching of the adhesion between poly(methyl methacrylate) brushes", ACS Macro Lett. 4, 75 (2015).

[9] Y. Yu, R. A. Lopez de la Cruz, B. D. Kieviet, H. Gojzewski, A. Pons, G. J. Vancso, and S. de Beer, "Pick up move and release of nanoparticles utilizing co-non-solvency of pnipam brushes", Nanoscale 9, 1670-1675 (2017), URL http://dx.doi .org/10.1039/C6NR09245D.

[10] Z. Cao, Q. Bian, Y. Chen, F. Liang, and G. Wang, "Lightresponsive janus-particle-based coatings for cell capture and release", ACS Macro Letters 6, 1124-1128 (2017), URL https://doi.org/10.1021/ acsmacrolett.7b00714.

[11] I. Tokareva, S. Minko, J. H. Fendler, and E. Hutter, "Nanosensors based on responsive polymer brushes and gold nanoparticle enhanced transmission surface plasmon resonance spectroscopy", Journal of the American Chemical Society 126, 15950-15951 (2004), URL https://doi.org/10. 1021/ja044575y, pMID: 15584714 . 
[12] H. Merlitz, G.-L. He, C.-X. Wu, and J.-U. Sommer, "Nanoscale brushes: How to build a smart surface coating", Phys. Rev. Lett. 102, 115702 (2009), URL https://link.aps.org/doi/10.1103/PhysRevLett.102. 115702 .

[13] L. I. Klushin, A. M. Skvortsov, A. A. Polotsky, S. Qi, and F. Schmid, "Sharp and fast: Sensors and switches based on polymer brushes with adsorption-active minority chains", Phys. Rev. Lett. 113, 068303 (2014), URL https://link .aps .org/doi/10.1103/PhysRevLett.113.068303.

[14] T. Jovanovic-Talisman, J. Tetenbaum-Novatt, A. S. McKenney, A. Zilman, R. Peters, M. P. Rout, and B. T. Chait, "Artificial nanopores that mimic the transport selectivity of the nuclear pore complex", Nature $\mathbf{4 5 7}$, 1023 (2009).

[15] M. Tagliazucchi, O. Peleg, M. Kröger, Y. Rabin, and I. Szleifer, "Effect of charge, hydrophobicity, and sequence of nucleoporins on the translocation of model particles through the nuclear pore complex", Proc. Natl. Acad. Sci. USA 110, 3363 (2013).

[16] Y. Zhang, R. A. Mulvenna, S. Qu, B. W. Boudouris, and W. A. Phillip, "Block polymer membranes functionalized with nanoconfined polyelectrolyte brushes achieve sub-nanometer selectivity", ACS Macro Letters 6, 726-732 (2017), URL https://doi.org/10.1021/acsmacrolett. $7 \mathrm{~b} 00278$.

[17] H. Yang, H. Zhu, M. M. R. M. Hendrix, N. J. H. G. M. Lousberg, G. de With, A. C. C. Esteves, and J. H. Xin, "Temperature-triggered collection and release of water from fogs by a sponge-like cotton fabric", Advanced Materials 25, 1150-1154 (2013), URL https://onlinelibrary. wiley.com/doi/abs/10.1002/adma.201204278

[18] X. Liu, Y. Li, J. Hu, J. Jiao, and J. Li, "Smart moisture management and thermoregulation properties of stimuli-responsive cotton modified with polymer brushes", RSC Adv. 4, 63691-63695 (2014), URL http://dx. doi.org/10.1039/C4RA11080C.

[19] A. Omar, B. A. A., and H. W. T. S., "Ucst wetting transitions of polyzwitterionic brushes driven by self-association", Angewandte Chemie International Edition 45, 1770-1774 (2006), URL https://onlinelibrary. wiley.com/doi/abs/10.1002/anie.200503264

[20] J. A. Howarter and J. P. Youngblood, "Self-cleaning and anti-fog surfaces via stimuli-responsive polymer brushes", Advanced Materials 19, 
3838-3843 (2007), URL https://onlinelibrary.wiley.com/doi/abs/ 10.1002/adma.200700156.

[21] M. E. R. Shanahan, "The influence of solid micro-deformation on contact angle equilibrium", Journal of Physics D: Applied Physics 20, 945 (1987), URL http://stacks .iop.org/0022-3727/20/i=7/a=018.

[22] A. Carré, J.-C. Gastel, and M. E. R. Shanahan, "Visco-elastic effects in spreading of liquids", Nature 379, 432-434 (1996).

[23] E. R. Jerison, Y. Xu, L. A. Wilen, and E. R. Dufresne, "Deformation of an elastic substrate by a three-phase contact line", Phys. Rev. Lett. 106, 186103 (2011), URL https://link.aps.org/doi/10.1103/ PhysRevLett.106.186103.

[24] T. Kajiya, A. Daerr, T. Narita, L. Royon, F. Lequeux, and L. Limat, "Advancing liquid contact line on visco-elastic gel substrates: stick-slip vs. continuous motions", Soft Matter 9, 454-461 (2013), URL http:// dx.doi.org/10.1039/C2SM26714D.

[25] L. A. Lubbers, J. H. Weijs, L. Botto, S. Das, B. Andreotti, and J. H. Snoeijer, "Drops on soft solids: free energy and double transition of contact angles", Journal of Fluid Mechanics 747, R1-12 (2014).

[26] Z. Cao and A. V. Dobrynin, "Polymeric droplets on soft surfaces: From neumann's triangle to young's law", Macromolecules 48, 443-451 (2015), URL https://doi .org/10.1021/ma501672p.

[27] J. Dervaux and L. Limat, "Contact lines on soft solids with uniform surface tension: analytical solutions and double transition for increasing deformability", Proceedings of the Royal Society of London A: Mathematical, Physical and Engineering Sciences 471 (2015).

[28] S. Karpitschka, S. Das, M. van Gorcum, H. Perrin, B. Andreotti, and J. H. Snoeijer, "Droplets move over viscoelastic substrates by surfing a ridge", Nature Communications 6, 7891 (2015).

[29] S. Karpitschka, A. Pandey, L. A. Lubbers, J. H. Weijs, L. Botto, S. Das, B. Andreotti, and J. H. Snoeijer, "Liquid drops attract or repel by the inverted cheerios effect", Proceedings of the National Academy of Sciences 113, 7403-7407 (2016), URL http://www.pnas.org/content/113/27/ 7403 . 
[30] B. Andreotti, O. Baumchen, F. Boulogne, K. E. Daniels, E. R. Dufresne, H. Perrin, T. Salez, J. H. Snoeijer, and R. W. Style, "Solid capillarity: when and how does surface tension deform soft solids?", Soft Matter 12, 2993-2996 (2016), URL http://dx.doi.org/10.1039/C5SM03140K.

[31] R. W. Style, A. Jagota, C.-Y. Hui, and E. R. Dufresne, "Elastocapillarity: Surface tension and the mechanics of soft solids", Annual Review of Condensed Matter Physics 8, 99-118 (2017), URL https://doi.org/10. 1146/annurev-conmatphys-031016-025326.

[32] Q. Xu, K. Jensen, R. Boltyanskiy, R. Sarfat, R. W. Style, and E. R. Dufresne, "Direct measurement of strain-dependent solid surface stress", Nature Communications 8, 555 (2017).

[33] J. Bico, E. Reyssat, and B. Roman, "Elastocapillarity: When surface tension deforms elastic solids", Annual Review of Fluid Mechanics 50, 629-659 (2018), URL https://doi.org/10.1146/ annurev-fluid-122316-050130.

[34] H. Liang, Z. Cao, Z. Wang, and A. V. Dobrynin, "Surface stresses and a force balance at a contact line", Langmuir 34, 7497-7502 (2018), URL https://doi.org/10.1021/acs.langmuir.8b01680.

[35] R. D. Schulman, M. Trejo, T. Salez, E. Raphael, and K. Dalnoki-Veress, "Surface energy of strained amorphous solids", Nature Communications 9, 982 (2018).

[36] F. Lequeux, L. Talini, E. Verneuil, G. Delannoy, and P. Valois, "Wetting of polymers by their solvents", The European Physical Journal E 39, 12 (2016), URL https://doi.org/10.1140/epje/i2016-16012-y.

[37] P. G. de Gennes, "Conformations of polymers attached to an interface", Macromolecules 13, 1069 (1980).

[38] P. G. Ferreira, A. Ajdari, and L. Leibler, "Scaling law for entropic effects at interfaces between grafted layers and polymer melts", Macromolecules 31, 3994-4003 (1998), URL https://doi.org/10.1021/ma9712460.

[39] J. H. Maas, G. J. Fleer, F. A. M. Leermakers, and M. A. Cohen Stuart, "Wetting of a polymer brush by a chemically identical polymer melt: Phase diagram and film stability", Langmuir 18, 8871-8880 (2002). 
[40] C. Pastorino, K. Binder, T. Kreer, and M. Müller, "Static and dynamic properties of the interface between a polymer brush and a melt of identical chains", The Journal of Chemical Physics 124, 064902 (2006), URL https://doi.org/10.1063/1.2162883.

[41] F. Léonforte and M. Müller, "Statics of polymer droplets on deformable surfaces", The Journal of Chemical Physics 135, 214703 (2011), URL https://doi.org/10.1063/1.3663381.

[42] G. S. Grest and K. Kremer, "Molecular dynamics simulation for polymers in the presence of a heat bath", Phys. Rev. A 33, 3628 (1986).

[43] K. Kremer and G. S. Grest, "Dynamics of entangled linear polymer melts: A molecular-dynamics simulation", The Journal of Chemical Physics 92, 5057-5086 (1990), URL https://doi.org/10.1063/1.458541.

[44] D. Mukherji, C. M. Marques, and K. Kremer, "Collapse in two good solvents, swelling in two poor solvents: defying the laws of polymer solubility?", Journal of Physics: Condensed Matter 30, 024002 (2018), URL http://stacks.iop.org/0953-8984/30/i=2/a=024002.

[45] S. de Beer and M. H. Müser, "Friction in (im-) miscible polymer brush systems and the role of transverse polymer tilting", Macromolecules $\mathbf{4 7}$, 7666 (2014).

[46] S. de Beer, L. I. S. Mensink, and B. D. Kieviet, "Geometry-dependent insertion forces on particles in swollen polymer brushes", Macromolecules 49, 1070-1078 (2016).

[47] Throughout the manuscript we will employ Lennard Jones units, with $\sigma$ being the unit of length, $\epsilon$ being the unit of energy and $\tau$ being the unit of time.

[48] W. Humphrey, A. Dalke, and K. Schulten, "Vmd - visual molecular dynamics", J. Molec. Graphics 14, 33 (1996).

[49] J. H. Weijs, A. Marchand, B. Andreotti, D. Lohse, and J. H. Snoeijer, "Origin of line tension for a lennard-jones nanodroplet", Physics of Fluids 23, 022001 (2011), URL https://doi.org/10.1063/1.3546008.

[50] W. J. Brittain and S. Minko, "A structural definition of polymer brushes", Polym. Sci., Part A: Polym. Chem. 45, 3505 (2007).

[51] I. Coluzza and J.-P. Hansen, "Transition from highly to fully stretched polymer brushes in good solvent", Phys. Rev. Lett. 100, 016104 (2008), URL https://link.aps.org/doi/10.1103/PhysRevLett.100.016104. 
[52] B. Zdyrko, V. Klep, and I. Luzinov, "Synthesis and surface morphology of high-density poly(ethylene glycol) grafted layers", Langmuir 19, 1017910187 (2003).

[53] S. Plimpton, "Fast parallel algorithms for short-range molecular dynamics", Journal of Computational Physics 117, 1 (1995).

[54] J. N. Israelachvili, Intermolecular and Surface Forces (Academic Press, Elsevier, USA) (2011).

[55] R. Descas, J.-U. Sommer, and A. Blumen, "Grafted polymer chains interacting with substrates: computer simulations and scaling", Macromol. Theory Simul. 17, 429 (2008).

[56] L. G. MacDowell and M. Müller, "Adsorption of polymers on a brush: Tuning the order of the wetting phase transition", The Journal of Chemical Physics 124, 084907 (2006), URL https://doi.org/10.1063/1. 2172597.

[57] P. J. Flory, Principles of Polymer Chemistry (Cornell University Press, Ithaca) (1953).

[58] D. R. Heine, G. S. Grest, and J. G. Curro, "Structure of polymer melts and blends: Comparison of integral equation theory and computer simulations", Advances in Polymer Science 173, 209 (2005).

[59] W. Zhang, E. D. Gomez, and S. T. Milner, "Predicting flory-huggins $\chi$ from simulations", Phys. Rev. Lett. 119, 017801 (2017), URL https: //link.aps.org/doi/10.1103/PhysRevLett.119.017801.

[60] M. A. Cohen Stuart, W. M. de Vos, and F. A. M. Leermakers, "Why surfaces modified by flexible polymers often have a finite contact angle for good solvents", Langmuir 22, 1722-1728 (2006), URL https://doi.org/ $10.1021 / 1 \mathrm{a} 052720 \mathrm{v}$

[61] T. Kreer, K. Binder, and M. H. Müser, "Friction between polymer brushes in good solvent conditions: steady-state sliding versus transient behavior", Langmuir 19, 7551 (2003).

[62] M. Aubouy, G. H. Fredrickson, P. Pincus, and E. Raphael, "End-tethered chains in polymeric matrixes", Macromolecules 28, 2979-2981 (1995), URL https://doi .org/10.1021/ma00112a051.

[63] G. S. Grest, "Grafted polymer brushes in polymeric matrices", The Journal of Chemical Physics 105, 5532-5541 (1996), URL https://doi.org/10. 1063/1.472395. 
[64] M. Aubouy and E. Raphael, "Surface-tethered chains in polymeric matrixes", Journal de Physique II 3, 443-448 (1993).

[65] G. Reiter and R. Khanna, "Negative excess interfacial entropy between free and end-grafted chemically identical polymers", Phys. Rev. Lett. 85, 5599-5602 (2000), URL https://link.aps.org/doi/10.1103/ PhysRevLett.85.5599.

[66] M. W. Matsen and J. M. Gardiner, "Autophobic dewetting of homopolymer on a brush and entropic attraction between opposing brushes in a homopolymer matrix", The Journal of Chemical Physics 115, 2794-2804 (2001), URL https://doi.org/10.1063/1.1385557.

[67] X. Zhang, F. K. Lee, and O. K. C. Tsui, "Wettability of end-grafted polymer brush by chemically identical polymer films", Macromolecules 41, 8148-8151 (2008), URL https://doi.org/10.1021/ma801549r.

[68] H. Lee, V. Sethuraman, Y. Kim, W. Lee, D. Y. Ryu, and V. Ganesan, "Nonmonotonic glass transition temperature of polymer films supported on polymer brushes", Macromolecules 51, 4451-4461 (2018), URL https : //doi.org/10.1021/acs.macromol.8b00290. 


\section{Chapter 4}

\section{The role of Entropy in Wetting of Polymer Brushes *}

The wetting of polymer brushes exhibits a much richer phenomenology than wetting of normal solid substrates. These brushes allow for three wetting states, which are partial wetting, complete wetting and mixing. Moreover, when wetted by chemically identical melts, entropic effects can cause brushes to display autophobicity. How entropy affects the transitions between the wetting states for brushes and chemically distinct melts is, however, not yet well-known, despite its relevance for potential applications. Here we present molecular dynamics simulations that reveal the role of entropic interactions in the transitions between the wetting states for polymer brushes wetted by polymer melts by comparing them to predictions based on enthalpic interactions alone. We show that entropy affects both the transition from mixing to demixing and the transition from complete to partial wetting. Enthalpically speaking, mixing would occur when the interactions between the brush and the melt is stronger than the average interactions within the brush and the melt, while partial wetting occurs whenever the interaction between brush and melt is weaker than internal melt interactions. We find that the entropic interactions can either facilitate or inhibit these wetting transitions, meaning that the transitions occur at smaller or larger brush-melt interactions than predicted from enthalpic arguments. These effects strongly depend on the melt chain lengths and to a smaller extent on the brush grafting densities. Long chains and high grafting densities cause negative entropic contributions, resulting in mixing and complete wetting transitions to occur only at strong brush-melt interactions. In contrast, short chains and low grafting densities result in positive entropic contributions that aid wet-

${ }^{*}$ To be submitted as: L.I.S. Mensink, S.J.A. de Beer and J. H. Snoeijer, The role of Entropy in Wetting of Polymer Brushes 
ting transitions such that they occur at brush-melt interactions that are weaker than predicted from enthalpy. Our results highlight the relevance of entropy in predicting the exact wetting transitions, which is important for the design of brush-based applications such as oleophobic and responsive self-cleaning surfaces.

\subsection{Introduction}

Polymer brushes consist of long macromolecules densely end-attached to a surface [1]. They are popular building blocks in the design of functional surface coatings due to their versatility and simplicity to manipulate. [2, 3] For example, these brushes have been employed as smart sensors [4 6] or to manage surface lubricity [7,9] and adherence. [10, 11] Surface wettability is also tuned straightforwardly with polymer brushes, which has resulted in the development of moisture management systems [12] and self-cleaning surfaces. [13] For these applications, it is important to understand the wetting of polymer brushes.

Wetting of polymer brushes deviates from wetting behavior on solid surfaces [14, 15]. While the solids are either partially or completely wetted, polymer brush coatings can also absorb liquids, which gives rise to three wetting states, partial and complete wetting and the mixed state [16]. The wetting state of the system is determined by the brush-liquid interactions and the stiffness of the brush (see figure 4.1). The three different states are separated by two transition-lines: the mixing to demixing transition (dashed) and the complete to partial wetting transition (dash-dot). To a large extent, these transitions can be understood using enthalpic arguments. When the affinity of the brush with the liquid, $\epsilon_{\mathrm{BL}}$, is less strong than the average self-affinities for the brush, $\epsilon_{\mathrm{BB}}$, and the liquid, $\epsilon_{\mathrm{LL}}$, the transition from mixing to demixing will occur. This happens when one goes from left to right crossing the gray dashed line, $W=\epsilon_{\mathrm{BB}}+\epsilon_{\mathrm{LL}}-2 \epsilon_{\mathrm{BL}}=0$, in figure 4.1. If the self-affinity of the liquid, $\epsilon_{\mathrm{LL}}$, is stronger than the affinity of the brush with the liquid, $\epsilon_{\mathrm{BL}}$, a transition from complete wetting to partial wetting can be observed. This happens when one goes from left to right, crossing the gray dash-dotted line. Though these enthalpic arguments capture the global behavior, there are clear deviations between the predicted (gray) and measured (black) transitions. To predict the measured transitions, entropy needs to be taken into account [16].

In this chapter, we will elucidate the role of entropy in the prediction of the wetting phase diagram of brushes wetted by polymeric nanodroplets. From early work on the wetting of brushes by chemically identical polymer melts, it is known that entropic effects can cause counter-intuitive phenomena such as demixing [14, 17, 22] between the brush and the melt. This effect has been 


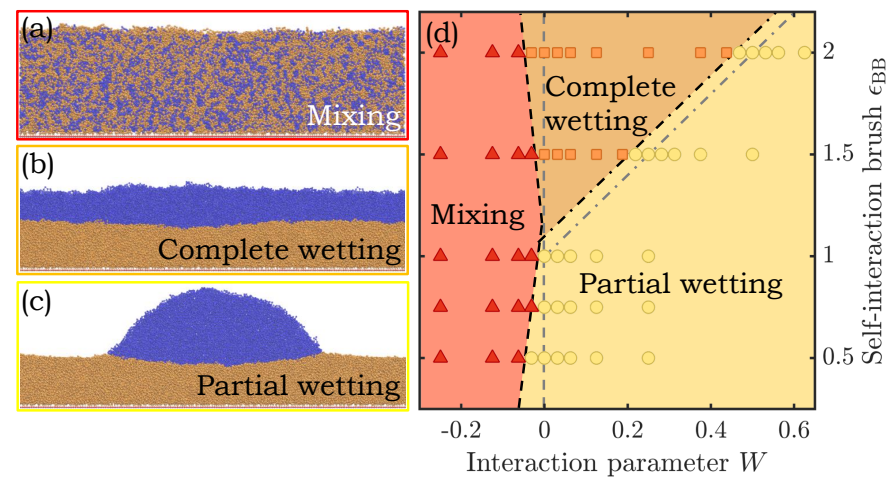

Figure 4.1: (a-c) Snapshots of simulation cells showing a polymer droplet (blue), interacting with a polymer brush (orange) for the three wetting states. (d) Interaction-based phase diagram depicting the relation between the states of wetting and brush self-interaction $\epsilon_{\mathrm{BB}}$ and the interaction parameter $W$. Observed are mixing (red triangles), complete wetting (orange squares) and partial wetting states (yellow circles). The gray lines indicate the enthalpic predictions for the mixing to demixing transition $\left(W=\epsilon_{\mathrm{BB}}+\epsilon_{\mathrm{LL}}-2 \epsilon_{\mathrm{BL}}=0\right.$, dashed) and the transition from complete to partial wetting $\left(\epsilon_{\mathrm{BL}}=\epsilon_{\mathrm{LL}}\right.$, dashdot). The black lines give the transitions observed in the MD simulations of reference 16 .

observed to strongly depend on the grafting density and the molecular weight of the melt and can result in autophobic dewetting [14, 21, 23, 24], where melt droplets are formed on the chemically identical brush.

First, to understand the phenomena of demixing, one needs to consider the different entropic contributions to the free energy [17]. Without end-grafting of brush-polymers, melt and brush-polymers would mix to gain translational entropy. However, since brush-polymers are constrained, they cannot gain translational entropy upon mixing. Instead, their entropy is reduced by mixing due to the elastic penalty for stretching upon absorption of the melt. Because brush stretching depends on the grafting density of the brush, the mixing to demixing transition depends on the grafting density as well [17, 18, 25]. Moreover, the translational entropy depends on the length of the melt-chains and, therefore, the mixing to demixing transition depends on the polymer length too [17, 18, 25].

Next, to understand autophobic dewetting, we have to consider the effect of entropy on the spreading parameter $S$. The spreading parameter is defined as $S=\gamma_{\mathrm{BV}}-\left(\gamma_{\mathrm{BL}}+\gamma_{\mathrm{LV}}\right)$, where $\gamma_{\mathrm{BV}}, \gamma_{\mathrm{BL}}$ and $\gamma_{\mathrm{LV}}$ are the interfacial tensions between the solid brush and vapor phase (BV), the brush and liquid melt phase (BL) and the melt and vapor phase (LV), respectively. When the spreading 
parameter is positive $(S>0)$, complete wetting occurs and the droplet will partially wet the surface when $S<0$.

The interfacial tension between chemically identical brushes and melts is inversely proportional to the difference in entropy before and after bringing the brush and melt in contact. For short melt polymers and low grafting densities, the change in entropy is positive, while it is negative for long melt polymers and high grafting densities. This implies that the interfacial tension $\gamma_{\mathrm{BL}}$ is positive for the latter conditions. For small positive values $\left(\gamma_{\mathrm{BL}}<\gamma_{\mathrm{BV}}-\gamma_{\mathrm{LV}}\right)$, the spreading parameter can be positive and complete wetting occurs. Only when the entropic penalty for mixing is large enough, $\gamma_{\mathrm{BL}}$ will be large enough such that $\gamma_{\mathrm{BL}}>\gamma_{\mathrm{BV}}-\gamma_{\mathrm{LV}}$ and partial wetting will occur.

The above described demixing and autophobicity effects are well characterized for brush-melt systems that are chemically identical. [17-22] Yet, for many applications, the brushes will be in contact with chemically distinct media. [2628. The role of entropy in wetting and, in particular, the wetting transitions for brush-melt systems of chemically distinct media is still largely unexplored and we address this in the present chapter. We study the role of entropy on the wetting transitions of polymer brushes wetted by polymer droplets, using MD simulations. The simulation method is described in section 2. Then, we provide an overview of the existing theoretical predictions in section 3 . The numerical results follow in section 4, where we first explore the effects of enthalpy and entropy at the mixing to demixing transition in section 4.1. Next, we reveal the influence of entropy on the partial wetting to complete wetting transition in section 4.2. Lastly, in section 4.3 we discuss the partial wetting regime, where we focus on the relation between contact angles and interfacial tension measurements using Kirkwood-Buff. In both wetting transitions entropy comes into play for chemically distinct brushes and melts, where positive entropic contributions are found for short melt chains and, in the case of the mixing transition, low grafting densities. Negative entropic effects were observed for long chains and high grafting densities, due to reduced translational entropy from the long melt chains and an increased entropic penalty for brush stretching.

\subsection{Model and Methods}

\subsubsection{Molecular Dynamics Simulations}

We use a coarse-grained bead-spring model (Kremer-Grest model [29]) to represent the polymers. This model is known to successfully describe the qualitative behavior of bulk polymers [30], polymers in solvent(-mixtures) [31] as well as polymer brushes [32, 33]. Non-bonded interactions within this model 
are described using a Lennard-Jones (LJ) potential:

$$
V_{\mathrm{LJ}}=4 \epsilon\left(\left(\frac{\sigma}{r}\right)^{12}-\left(\frac{\sigma}{r}\right)^{6}\right) \quad\left(r<r_{\mathrm{c}}\right),
$$

using $\sigma=1$ and a cut off length $r_{\mathrm{c}}=2.5 \sigma$.

Through this chapter, we will employ Lennard-Jones units in which $\sigma$ and $\epsilon$ define the units of length and the unit of energy, respectively. Considering for example poly(ethylene), these units can be converted to real values for the polymers using $\epsilon=30 \mathrm{meV}$ and $\sigma=0.5 \mathrm{~nm}$ [30]. We use a finite extensible nonlinear elastic (FENE) potential combined with the repulsive part of the Lennard-Jones potential to describe the bonded interactions between consecutive beads within the polymer chains:

$$
V_{\mathrm{KG}}=-0.5 k R_{0}^{2} \ln \left[1-\left(\frac{r}{R_{0}}\right)^{2}\right]+4 \epsilon\left[\left(\frac{\sigma}{r}\right)^{12}-\left(\frac{\sigma}{r}\right)^{6}\right]+\epsilon .
$$

For $V_{\mathrm{KG}}$, the LJ interaction parameters are $\epsilon=1, \sigma=1$ and $r_{\mathrm{c}}=2^{1 / 6} \sigma$ and we use a stiffness of $k=30 \epsilon / \sigma^{2}$ and a maximum extension $R_{0}=1.5 \sigma$, which ensures that there is no bond-crossing [29]. One polymer bead represents typically 3-4 monomers and, thus, the unit of mass $[\mathrm{m}]$ is $10^{-22} \mathrm{~kg}$ and the unit of time $[\tau]$ represents $0.3 \mathrm{~ns}$ [30].

Figure 4.2(a) shows a typical snapshot of our simulation cell. The snapshots are rendered using VMD [34]. Our simulation cells consist of a wall composed of single layer of Lennard-Jones particles (red) in a triangular lattice with polymers (orange) anchored by one end to the wall particles. Each individual brush-polymer consists of $N_{\mathrm{B}}=100$ repeat units. The density at which these polymers are attached to the surface is varied as $\sigma_{\mathrm{GD}}=0.067,0.15,0.2,0.27$ (chains $/ \sigma^{2}$ ), which equals approximately $1.3,3,4$ and 5.3 times the critical grafting density for brush formation under poor solvent conditions. This means our simulations are performed in the brush regime, which is also apparent from the snapshots and density profiles given in figure 4.2(b). They show a step-wise change in the brush density, which is clearly different from the strong variation in density observed for the so-called mushrooms that are formed at densities below the critical grafting density [35]. The polymer brush is in contact with a uniform polymer melt with a constant chain length that is varied between $N=2$ and $N=64$ for individual measurements. The total number of beads present in the polymer melt is kept constant, so the amount of molecules wetting the brush is varied (for $N=2$ there are 8000 melt molecules, for $N=64$ we simulated 250 melt molecules).

Our simulations are performed at a constant box-size and volume $V$, inside a quasi-2D setup, as shown in figure 4.2(a). Our simulation box is periodic in 
(a)

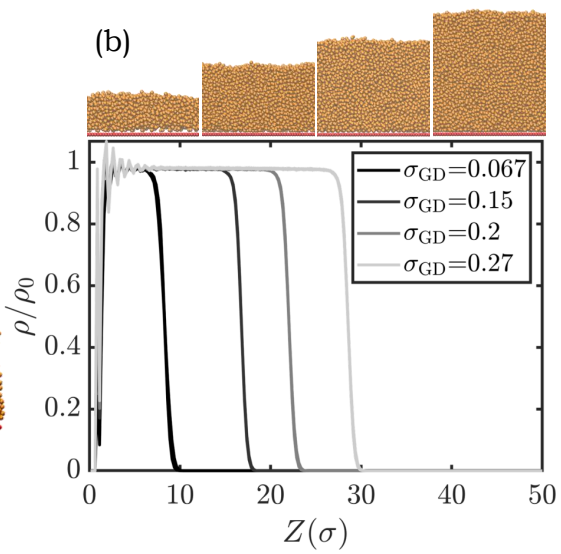

Figure 4.2: (a) A typical snapshot of a quasi-2D simulation set-up. Shown is a polymer droplet (blue) partially wetting a polymer brush (orange). (b) A graph showing the polymer brush density profile for polymer chains grafted at different grafting densities $\left(\sigma_{\mathrm{GD}}=0.067,0.15,0.2,0.27\left(\right.\right.$ chains $\left.\left./ \sigma^{2}\right)\right)$. Snapshots show corresponding brushes.

$x$ and $y$, with a boxlength in $x$ of at least $108 \sigma$, depending on the spreading of the droplet. For small contact angles, we increased the boxsize in $x$ to 218 $\sigma$. The boxlength in $y$ is limited to $15 \sigma$ to suppress Rayleigh instabilities in the infinitely long cylindrical droplet. Yet, it is large enough to ensure that there are no self-interactions between polymer chains. Moreover, in this setup line-tension effects are prevented [36].

The equations of motion are solved using the velocity Verlet algorithm as implemented in LAMMPS [37], using a timestep of $\Delta t=0.005 \tau$. The simulations are performed in the $N V T$ ensemble. The temperature $T$ is kept constant at $k_{\mathrm{B}} T=1 \epsilon\left(k_{\mathrm{B}}\right.$ being the Boltzmann constant) using a Langevin thermostat with a damping coefficient of $\xi=1 \tau^{-1}$. The simulation cell are equilibrated for $10^{7}$ timesteps before the production runs of $10^{7}$ timesteps during which the observables are extracted.

From the production runs we extract the density of the brush and the melt as a function of the distance from the wall in slabs of $0.2 \sigma$. From these density profiles overlap integrals are calculated, which equal the product of the brush and the melt density integrated over the distance [38]. These overlap integrals are employed to determine whether the brush and the melt have mixed or not. For the mixed state, the overlap integral will reach a maximum value. From this maximum overlap value, we determine the mixing to demixing transition to occur at $50 \%$ of this maximum. We also compute the contact angle of the melt droplet from the production runs, by fitting spherical caps to the 
isodensity contours of the droplet, as previously reported [36.

To tune the affinity between the brush and the melt, relative to the selfinteraction of the brush, we alter the strength of the Lennard-Jones potential via $\epsilon$. We study two different strengths for the self-interaction of the brush $\epsilon_{\mathrm{BB}}$, namely $\epsilon_{\mathrm{BB}}=1$ and $\epsilon_{\mathrm{BB}}=1.5$. When $\epsilon_{\mathrm{BB}}$ is high enough, polymer brushes are attracted to one another, resulting in a collapsed polymer brush structure. We checked that for these $\epsilon_{\mathrm{BB}}$ 's, the bare brushes are indeed in the collapsed state (see appendix figure 4.18). This is in contrast to Milchev et al. [39, who studied brush-wetting for low $\epsilon_{\mathrm{BB}}$ and, thus, (implicit) good solvent conditions. Similarly to soft elastomers [40, 41, brushes can deform due to surface tension and form wetting ridges. [42]. Due to our choice of $\epsilon_{\mathrm{BB}}$, the brushes are relatively hard and wetting ridges are small and do not affect the macroscopic contact angle [16. We vary the interaction between the polymer brush and polymer melt $\epsilon_{\mathrm{BL}}$ between 0.375 and 1.5 , while $\epsilon_{\mathrm{LL}}=1$ is kept constant, so that we move through the interaction-based phase diagram from figure 4.1(d) along horizontal lines. From the simulations, we aim to extract generic relations and we do not intend to model particular types of polymers. We allow all sorts of interactions and do not limit this to van der Waals potentials. Therefore, mixing rules do not apply [43] and we can vary $\epsilon_{\mathrm{BB}}$ and $\epsilon_{\mathrm{BL}}$ independently from each other. The interactions between the wall and the polymer- and liquid-beads is kept purely repulsive $(\epsilon=1, \sigma=1$ and $r_{\mathrm{c}}=2^{1 / 6} \sigma$ ) to prevent preferential adsorption near the wall [44] and higher order wetting transitions. [45]

\subsection{Theory}

In this section we review the theories that have been developed to predict the wetting of polymer brushes by polymer melts. These will be used for comparison to the molecular dynamics simulations in section 4 .

\subsubsection{Mixing-Demixing}

To predict when a chemically distinct brush and a melt will mix, we will first consider the mixing free energy for chemically identical brush-melt systems. In our explanation, we follow the arguments of Raphaël and coworkers, [17, 18, 25] which is based on a Flory-type approach using a constant number of particles $N$, constant volume $V$ and constant temperature $T$, such that the Helmholtz free energy $F=U-T S$ will be minimized. Next, we will regard an extended model by including the interactions between chemically distinct brushes and melts, for which we follow the arguments of Zhulina and coworkers. 46] 


\section{Chemically Identical Brush and Melt}

When the brush and the melt are chemically identical, the mixing to demixing transition is determined by entropic interactions alone. Under these conditions, the free energy is given by: [18]

$$
\frac{F}{k_{\mathrm{B}} T}=\frac{L^{2}}{a^{2} N_{\mathrm{B}}}+\frac{a^{3}}{N} \frac{N_{\mathrm{B}}^{2}}{L D^{2}},
$$

where $L$ is the height of the brush, $a$ is the effective monomer size and $D$ is the distance between the anchorpoints given by $D=a \sigma_{\mathrm{GD}}^{-1 / 2}$. The first term of equation 4.3 represent the entropic penalty that the polymers in the brush pay upon stretching. The second term arises due to excluded volume interactions between the polymer- and/or melt-beads in the system. From equation 4.3 a geometry-based phase diagram can be constructed that displays for what system parameters the brush and melt will be mixing (region 1) or non-mixing (regions 2 and 3), as shown in figure 4.3 . The boundary between the mixed and the demixed state can be found by minimizing equation 4.3 with respect to the height of the brush $L$, resulting in:

$$
L \propto a N_{\mathrm{B}} N^{-1 / 3} \sigma_{\mathrm{GD}}^{1 / 3}
$$

As expected, higher grafting densities result in higher, more stretched polymer brushes. Moreover, upon increasing $N$, the brush height decreases. When employing that the brush-density of a dry brush is of order one and given by $\rho=N_{\mathrm{B}} \sigma_{\mathrm{GD}} a^{3} / L$, we can substitute equation 4.4 to obtain the boundary between dry unmixed and mixed brushes to be:

$$
\sigma_{\mathrm{GD}, \operatorname{tr}} \propto N^{-\frac{1}{2}}
$$

where $\sigma_{\mathrm{GD} \text {,tr }}$ is the transition grafting density above which the melt and the brush demix. This equation is shown in figure 4.3 depicting the transition between mixing in region (1) and demixing in region (2) for $N<N_{\mathrm{B}}$. For $N>N_{\mathrm{B}}$, the transition between mixing in region (1) and demixing in region (3) is a horizontal line described by $\sigma_{\mathrm{GD}, \mathrm{tr}} \equiv N_{\mathrm{B}}^{-1 / 2}$. At this transition, the brush alters from a sparse brush into a dense brush, thus excluding the melt chains. As intuitively expected, brushes and melts demix only at high grafting densities for short melt chains, while for large melt chains demixing occurs already at much lower grafting densities.

\section{Chemically Distinct Brush and Melt}

To predict the mixing-demixing transition for chemically distinct melts and brushes, we have to consider enthalpic interactions on top of the entropic in- 


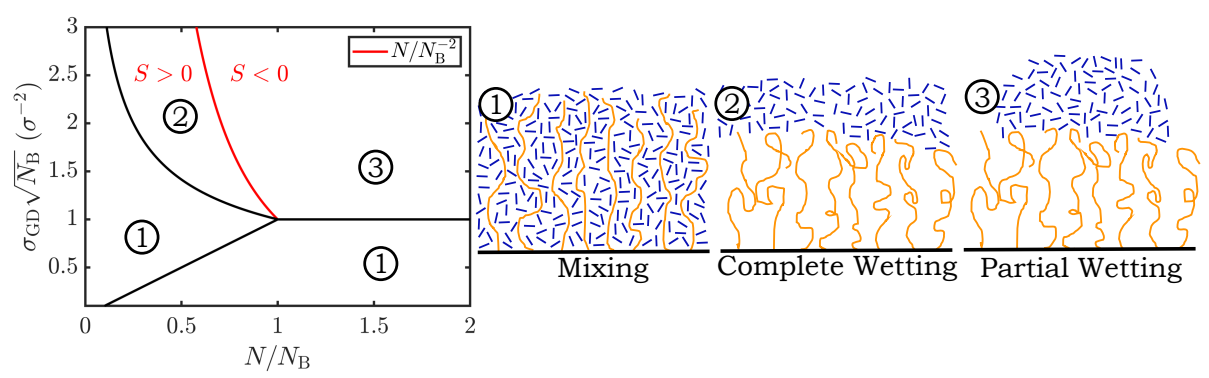

Figure 4.3: A geometry-based phase diagram showing the different states of mixing (1), complete wetting (2) and partial wetting (3) for a grafted polymer - polymer melt system. The phase diagram depicts the relation between the wetting states and the grafting density $\sigma_{\mathrm{GD}}$ and the melt chain length $N$. Here, $N_{\mathrm{B}}$ is the polymer brush chain length. The mixing to demixing transition (region (1) to (2)) is described by equation 4.5. The transition from complete to partial wetting (region (2) to (3)) is given by the red line (equation 4.9). In this figure, $S$ denotes the spreading parameter as defined by equation 4.8 . The three sketches on the right correspond to the configurations of the grafted polymers in each regime.

teractions considered in section 3.1.1. These enthalpic interactions are incorporated using the Flory Huggins interaction parameter $\chi=\frac{z}{2 k T}\left(\epsilon_{\mathrm{BB}}+\epsilon_{\mathrm{LL}}-2 \epsilon_{\mathrm{BL}}\right)$, with $z$ being the average number of neighbors of a polymer unit. This parameter is proportional to our interaction parameter $W$. Due to the enthalpic interactions, the free energy of the brush is now described by: [18]

$$
\frac{F}{k_{\mathrm{B}} T}=\frac{L^{2}}{a^{2} N_{\mathrm{B}}}+\frac{a^{3}}{N}(1-2 N \chi) \frac{N_{\mathrm{B}}^{2}}{L D^{2}} .
$$

Only positive excluded volumes are allowed such that $(1-2 N \chi) / N$ should remain positive. Since equation 4.3 and equation 4.6 can be directly converted into each other via the substitution $N \leftrightarrow N /(1-2 N \chi)$, the transition between mixing and demixing can also be obtained by the same substitution, which results in:

$$
\sigma_{\mathrm{GD}, \operatorname{tr}} \propto\left(\frac{(1-2 N \chi)}{N}\right)^{\frac{1}{2}} .
$$

Using equation 4.7, the $\chi$-dependent geometry-based phase diagrams can be straightforwardly constructed. The result is given in figure 4.4 showing sensitivity to small changes of $\chi$. Upon increasing $\chi$, the transition shifts to smaller $N$. 


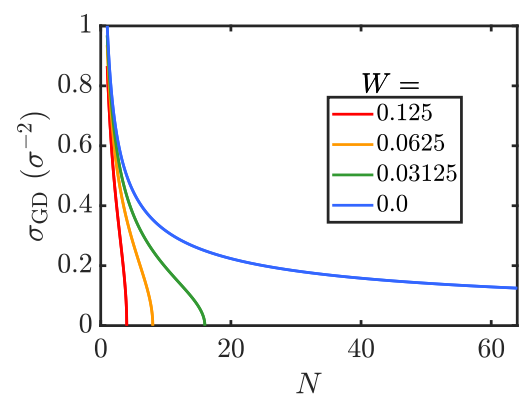

Figure 4.4: The transition grafting density as a function of the length of the melt polymers for different $W$, as predicted by equation 4.7 .

\subsubsection{Complete to Partial Wetting}

The transition from complete to partial wetting occurs when the spreading parameter $S=0$. For $S<0$, partial wetting is observed, while for $S>0$ liquids completely wets the surface. The spreading parameter for liquids on a polymer brush was first determined by Halperin and de Gennes [14 and is defined as: 47

$$
S=\gamma_{\mathrm{BV}}-\left(\gamma_{\mathrm{BL}}+\gamma_{\mathrm{LV}}\right)-\Delta f_{\mathrm{ch}} \sigma_{\mathrm{GD}},
$$

where $\gamma_{\mathrm{BV}}, \gamma_{\mathrm{BL}}$ and $\gamma_{\mathrm{LV}}$ are the brush-vapor, brush-liquid and liquid-vapor interfacial tensions, respectively. The last term in equation 4.8 gives the difference in free energy per chain $\Delta f_{\text {ch }}$ upon mixing compared to dry conditions, multiplied by the grafting density $\sigma_{\mathrm{GD}}$.

To predict the change in free energy per chain upon mixing of the chemically identical brush-melt system and, thereby, the transition from complete to partial wetting for these melts and brushes, Leibler et al., [48] utilized the strong stretching theory of Semonov and Milner-Witten-Cates. [49, 50] In this model the polymer density decays parabolically as a function of the distance from the surface, in contrast to the step function assumed in earlier brushmodels. [17, 51] Leibler et al. used the strong stretching theory to derive an expression for the penetration length into a brush, which determines the effective interfacial tension acting between the brush and air or the melt. [52 From this, it was shown that partial wetting occurs when $N>N_{\mathrm{B}}^{2 / 3} \sigma_{\mathrm{GD}}^{-2 / 3}$, while complete wetting would be observed for $N$ smaller than this boundary. Later, it was shown via more careful numerical self-consistent field theory (SCFT) [20], that

$$
N_{\mathrm{tr}} \propto \sigma_{\mathrm{GD}}^{-2}
$$


for very long $N_{\mathrm{B}}$ and that there is no universal scaling for arbitrary polymer lengths. 53] Experimental observations are, however, not able to distinguish the difference between these theories. [21, 23] In figure 4.3, we use equation 4.9 to denote the phase transition from complete to partial wetting.

\subsection{Results and discussion}

\subsubsection{Mixing - demixing}

First, we discuss the results of the MD simulations, addressing the mixing to demixing transition. Along the lines of the geometry-based phase diagrams of figure 4.3 and 4.4 , we varied the grafting density and melt polymerization, for varying values of $W$ and for two values of $\epsilon_{\mathrm{BB}}$. From these simulations, we first determine whether the system is in a mixing or demixing state. This was done using the overlap integral of density profiles for the brush and the polymer melt, which is defined as: $N_{\mathrm{ov}}=\int_{0}^{\infty} \rho_{\mathrm{A}}(z) \rho_{\mathrm{B}}(z) d z$, with $\rho_{\mathrm{A}}$ and $\rho_{\mathrm{B}}$ representing the densities of phase $\mathrm{A}$ and $\mathrm{B}$. Here, a high overlap integral means that mixing occurs, whereas a low overlap integral indicates demixing. By taking the overlap integral for several values of $W$, data-sets were gathered for constant values of $N$ and $\sigma_{\mathrm{GD}}$. These data-sets were then interpolated to determine whether each data point was above or below the transition-point. The resulting six geometry-based phase diagrams are given in figure 4.5. The different phase diagrams from left to right display the results for varying values for the interaction parameter $W$, and from top to bottom the brush-brush interaction strength $\epsilon_{\mathrm{BB}}$. These geometry-based phase diagrams illustrate the shift in the mixing to demixing transition for a melt wetting a brush, when compared to the chemically identical wetting case at $W=0$, as predicted by the theory in section 3.1. The top row shows the mixing transition for $\epsilon_{\mathrm{BB}}=1.0$ and the bottom row shows $\epsilon_{\mathrm{BB}}=1.5$. In the left and right columns, we show the effect of a shift in the interaction parameter to an increased and a decreased net attraction $(\Delta W=0.0625)$. From the top row $\left(\epsilon_{\mathrm{BB}}=1.0\right)$, we see that for $W=-0.0625$ (top left), only mixing occurs for our settings. These results are in qualitative agreement with previous observations [54]. Only for very high grafting densities and melt chain lengths, which are not studied in the present work, one would expect to see a transition for such a negative interaction parameter. For $W=0$ (top middle), we see a mixing to demixing transition. This transition follows the entropic predictions, as the brush and melt are chemically identical and thus the enthalpic contribution to the mixing energy is zero. For $W=0.0625$ (top right), the mixing to demixing transition has shifted to lower $N$ and $\sigma_{\mathrm{GD}}$, according to the theory for a chemically distinct brush and melt (equation 4.7), as shown in figure 4.4 . 

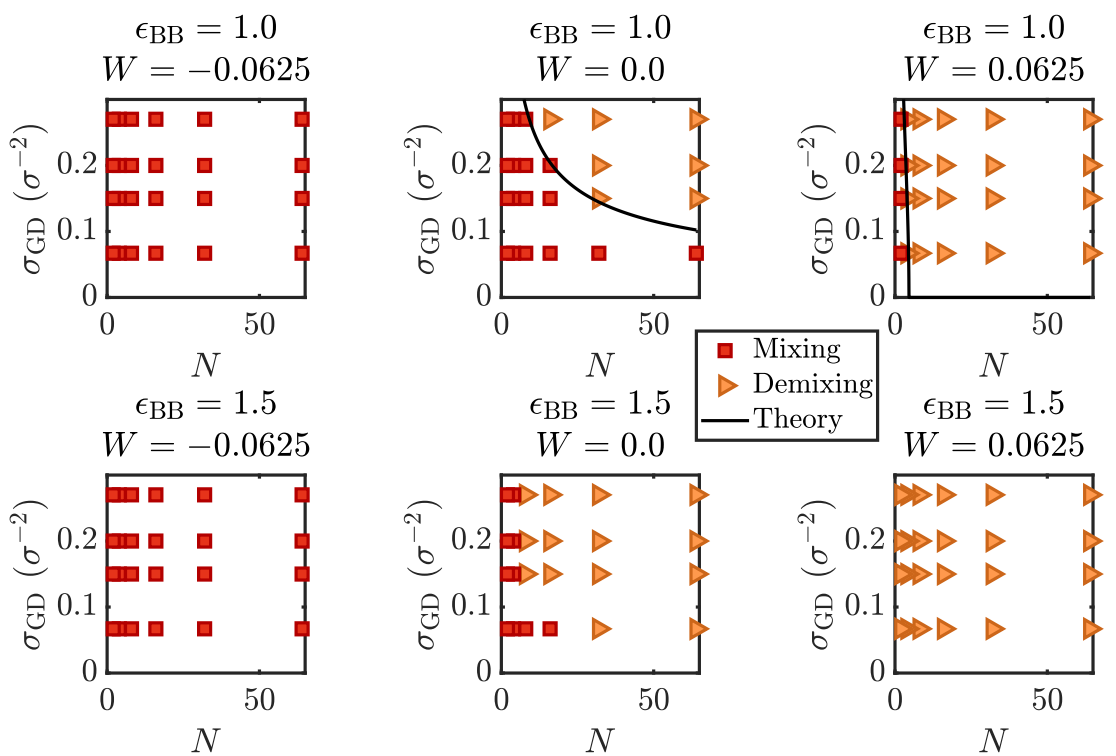

Figure 4.5: Geometry-based phase diagrams showing the influence of the grafting density $\left(\sigma_{\mathrm{GD}}\right)$ versus the degree of polymerization of the melt $(N)$, on the mixing-demixing behavior of the brush-melt system. The top row is measured for brush interactions $\epsilon_{\mathrm{BB}}$ of 1.0 , the bottom row is at $\epsilon_{\mathrm{BB}}=1.5$. The left column shows the phase diagram for an interaction parameter $W$ of -0.0625 , the middle for 0.0 , and the right column at 0.0625 .

This shift can be attributed to the higher degree of attractive interactions between brush and melt for higher values of $W$, meaning that mixing is more likely to occur. In the bottom row $\left(\epsilon_{\mathrm{BB}}=1.5\right.$, we observe no phase transitions for $W=-0.0625$ (bottom left), where we observe only mixing, and for $W=$ 0.0625 (bottom right), where we instead observe only demixing. For $W=0$, (bottom middle) we observe a phase-transition similar to the one for $W=0$ and $\epsilon_{\mathrm{BB}}=1.0$, but shifted downward, implying that demixing occurs for shorter chains compared to $\epsilon_{\mathrm{BB}}=1.0$. From this we can conclude that the free energy cost to stretch the brush polymers has increased, which is consistent with the stronger interaction between the brush polymers. Through the phase diagrams with a phase transition present (middle column and top right), we fitted the theoretical prediction (equation 4.7) of the mixing to demixing transition. For chemically identical brush and melt, we introduced a prefactor $a$, which was utilized as a fitting parameter. This fitting parameter can be related to the effective monomer size and we found it to equal $a=0.82$. For the chemically distinct system $(W=0.0625)$, we introduced a second fitting parameter to 
correct for the difference between our interaction parameter $W=\frac{1}{2}\left(\epsilon_{\mathrm{BB}}+\right.$ $\left.\epsilon_{\mathrm{LL}}\right)-\epsilon_{\mathrm{BL}}$ and the definition of the Flory Huggins parameter $\chi=\frac{z}{2 k T}\left(\epsilon_{\mathrm{BB}}+\right.$ $\left.\epsilon_{\mathrm{LL}}-2 \epsilon_{\mathrm{BL}}\right)$, resulting in a fit of the form: $\sigma_{\mathrm{GD}, \mathrm{tr}}=a((1-2 N b \chi) / N)^{\frac{1}{2}}$. From the data obtained for $W=0.0625$, we obtained $a=0.82$ and $b=1.75$. It is clear that the numerical data are well described by the dependency of equation 4.7 .

From the geometry-based phase diagrams we see that, for both values of $\epsilon_{\mathrm{BB}}$, a shift in $W$ strongly changes the mixing to demixing transition point, as was predicted by Zhulina, Aubouy and others [18, 46] (equation 4.7). This prediction, however, is strictly speaking only valid in the case of positive excluded volume interactions, meaning that $(1-2 N \chi)>0$. For our setting of $W=0.0625$, this is the case for $N=8,4$ and 2. Still, for the theoretical prediction at $\epsilon_{\mathrm{BB}}=1.0$ in figure 4.5 , this prediction remains consistent with our numerical results.

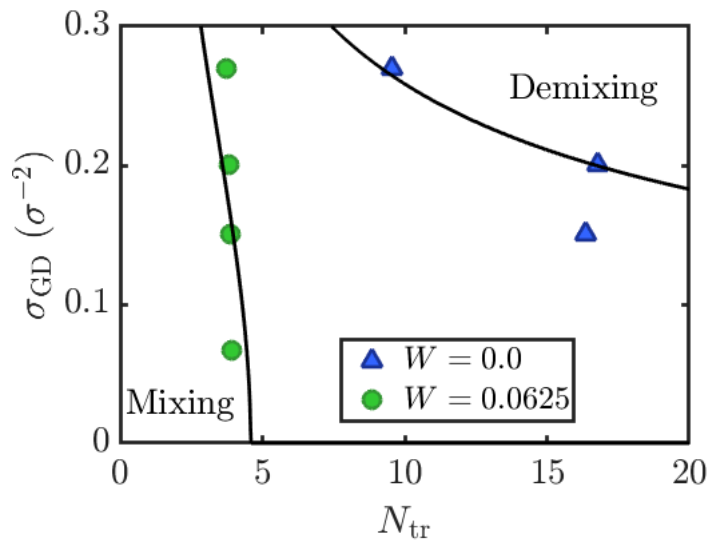

Figure 4.6: Melt chain lengths $N_{\mathrm{tr}}$ at which the mixing to demixing transition occurs, measured from the overlap integral, versus the grafting density $\sigma_{\mathrm{GD}}$, for the cases $W=0$ and $W=0.0625$.

In order to more accurately compare our results to theoretical predictions than we did in figure 4.5, we will now determine the exact $N$ at which our transition from mixing to demixing occurs. From the interpolations of the overlap integrals, we can extract the exact degree of melt polymerization at which this transition, $N_{\mathrm{tr}}$, occurs. These transition points are shown in figure 4.6, and are again fitted with equation 4.7, with the same prefactors as in figure 4.5. As expected, the $N_{\text {tr }}$ increases for lower grafting densities. The reason for this is the entropic penalty that the brushes pay, versus the increase in translational entropy for the polymer melt. In the case of long chains, this translational entropy is smaller, and in the case of densely grafted brushes, the 
entropic penalty of the brush becomes larger. Fitted through these data-sets is the theoretical prediction of the mixing to demixing transition at $W=0$ and $W=0.0625$, that is also shown in figure 4.3 . The data shows agreement to the behavior as predicted from theory for $W=0$.

Next, we would like to discuss how variations in $\sigma_{\mathrm{GD}}$ and $N$ influence the interaction-based phase diagram of figure 4.1, where the black dashed line indicates the $W_{\text {tr }}$ for mixing to demixing at $N=32$. Therefore, we determined the transition point in the interaction parameter $W_{\mathrm{tr}}$, for varying values of the polymer melt and the grafting density, and the results are presented in figure 4.7. The gray dashed lines describe the enthalpy-based transition $W_{\mathrm{tr}}$. In
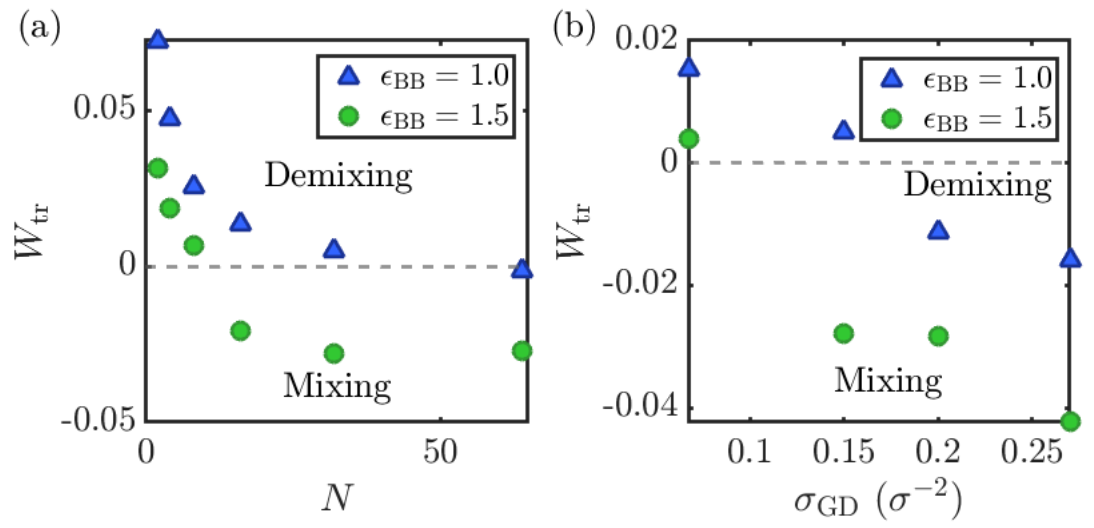

Figure 4.7: The interaction parameters $W_{\mathrm{tr}}$ at which the mixing to demixing transition occurs, as measured from the overlap integral, (a) for a defined melt length $N$, and (b) grafting density $\sigma_{\mathrm{GD}}$. The gray dashed lines in both figures describe the enthalpy-based transition.

figure 4.7 (a), $W_{\text {tr }}$ is shown as a function of $N$. For small chains, the transition occurs at $W>0$, meaning that entropy is gained by mixing. In contrast, the longer melt chains show a shift to negative $W_{\text {tr }}$, because long chains gain less translational entropy than shorter chains. This smaller amount of entropy gain is not enough to compensate for the entropic penalty to stretch the polymer brush in order to mix. Figure 4.7(b) depicts $W_{\mathrm{tr}}$ for varying $\sigma_{\mathrm{GD}}$, indicating a shift to positive $W_{\text {tr }}$ for lower grafting density brushes, and a lower, negative $W_{\text {tr }}$ for higher grafting densities. This effect of the grafting density is caused by densely grafted brushes having to pay more of an entropy penalty for stretching upon mixing, as compared to less densely grafted brushes.

In summary, when regarding only enthalpic interactions, the mixing to demixing transition occurs at $W=0$. Entropic interactions can increase or decrease $W_{\mathrm{tr}}$, depending on $N$ and $\sigma_{\mathrm{GD}}$, where shorter chains and lower 
grafting densities tend to increase $W_{\text {tr }}$, meaning that entropy is gained from mixing. Our measurements for both chemically identical and chemically distinct brushes and melts, show good agreement to theoretical predictions from equation 4.7 on the occurrence of the mixing to demixing transition. We have compared this theoretical transition both to a direct geometry-based phase diagram of our system, and to exact predictions of this transition, based on our data.

\subsubsection{Partial wetting - complete wetting}
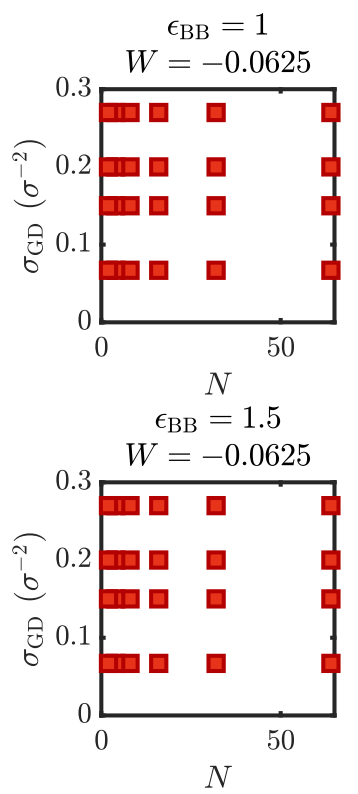
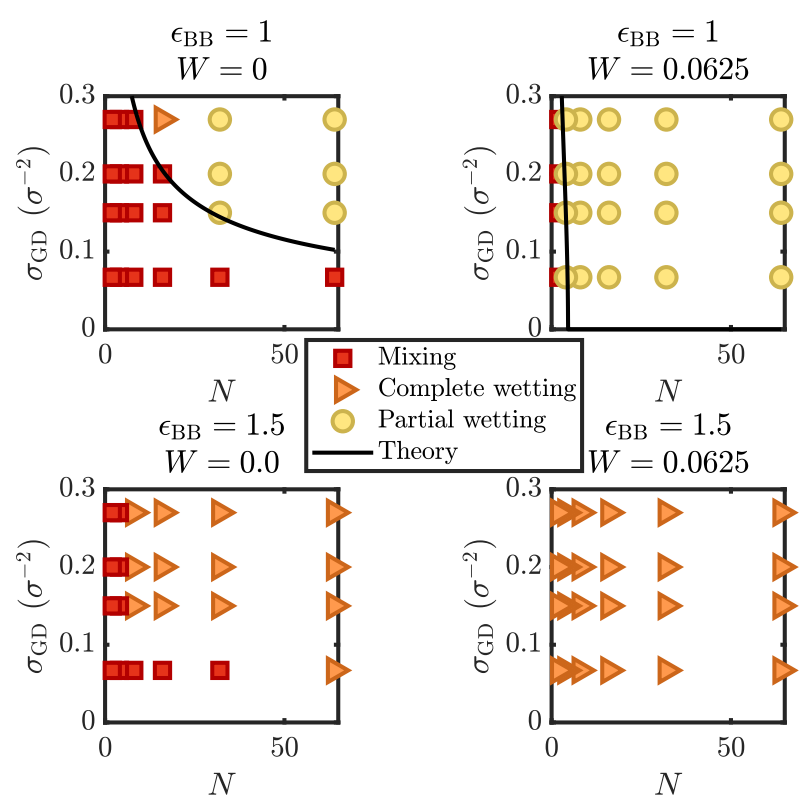

Figure 4.8: Geometry-based phase diagrams showing the influence of the grafting density $\left(\sigma_{\mathrm{GD}}\right)$ versus the degree of polymerization of the melt $(N)$, on the partial - complete wetting behavior of the brush-melt system. The top row is measured for brush interactions $\epsilon_{\mathrm{BB}}$ of 1.0, the bottom row is at $\epsilon_{\mathrm{BB}} 1.5$. The left column shows the phase diagram for an interaction parameter $W$ of -0.0625 , the middle for 0.0 , and the right column at 0.0625 .

We now focus on the transition from partial wetting to complete wetting. We show geometry-based phase diagrams in figure 4.8 indicating whether the system is partially wetting, complete wetting, or mixing. As in figure 4.5, the interaction parameter $W$ is varied from -0.0625 , to 0 to 0.0625 from left to right. The top row shows phase diagrams for $\epsilon_{\mathrm{BB}}=1.0$, the bottom row for $\epsilon_{\mathrm{BB}}=1.5$. The phase transitions in the top middle and top right diagrams were 
fitted using the same theory and fitting parameters as in figure 4.5, meaning that we consider the mixing to demixing theory. In the case of $\epsilon_{\mathrm{BB}}=1.0$ we see a transition directly from mixing to partial wetting for all grafting densities except the highest, where a complete wetting state is observed. For $\epsilon_{\mathrm{BB}}=1.5$ we observe a transition from mixing to complete wetting. We also expect to observe a complete to partial wetting transition for $\epsilon_{\mathrm{BB}}=1.5$, but for higher values of $W$, as is also illustrated in figure 4.1. This difference in transitioning from mixing to partial wetting or to complete wetting for the different $\epsilon_{\mathrm{BB}}$ 's, is caused by the stronger interaction within the brush for $\epsilon_{\mathrm{BB}}=1.5$. As the brush-brush interactions are stronger at $\epsilon_{\mathrm{BB}}=1.5$, the brush is more collapsed than a brush at $\epsilon_{\mathrm{BB}}=1.0$, resulting in a higher surface tensions $\gamma_{\mathrm{BL}}$ and $\gamma_{\mathrm{BV}}$ (see also the appendix 4.6.2 for values of $\Upsilon_{\mathrm{BV}}$ ). A higher increase for $\gamma_{\mathrm{BV}}$ than for $\gamma_{\mathrm{BL}}$ causes the spreading parameter to become higher and as such, facilitates a transition to complete wetting.

By regarding the transitions present in figure 4.3 , we expect mixing to occur for small values of $N$ such that $\sigma_{\mathrm{GD}, \mathrm{tr}}<N^{-1 / 2}$ (region 1), complete wetting for slightly larger $N$ (region 2), and partial wetting to occur at the largest values of $N$, where $\sigma_{\mathrm{GD}, \mathrm{tr}}>N / N_{\mathrm{B}}^{-2}$ (region 3 ). Therefore, we would expect to observe a larger complete wetting phase area in between the mixing and partially wetting phases for $\epsilon_{\mathrm{BB}}=1.0$ and $W=0$ and 0.0625 . However, it is difficult to clearly define the wetting state of systems that are close to a wetting transition, as illustrated in figure 4.9. Here, we show density profiles and snapshots with increasing melt length $N$, for a grafting density of $\sigma_{\mathrm{GD}}=0.27$. The density profile reveals a melt-rich phase at the top of the brush that, for increasing $N$, grows in concentration at the cost of the concentration of melt in the polymer brush bulk phase. For our definition of mixing and demixing states, we used interpolation of the overlap integrals, as mentioned in section 4.1. To distinguish between partial and complete wetting, we use visual inspection to assign wetting layers of homogeneous thicknesses to the complete wetting regime, and wetting layers of inhomogeneous thicknesses to the partially wetting regime. It is evident from the melt-rich phase at the top however, that this definition for mixing and demixing phases might be insufficient at accurately categorizing mixing phases and complete wetting phases. The snapshots beside the density profile show mixing, complete wetting and partial wetting points that are close to the wetting transition, where the pictures for $N=8,16$ and 32 display behavior that is not visibly clear to determine as either mixing, complete wetting or partially wetting. Around this point, the system can also be considered to be in the complete wetting state. For example, in the case where $N=32$, we observe a thin layer of melt wetting the entire brush, yet we find the layer of polymer melt to be thicker 

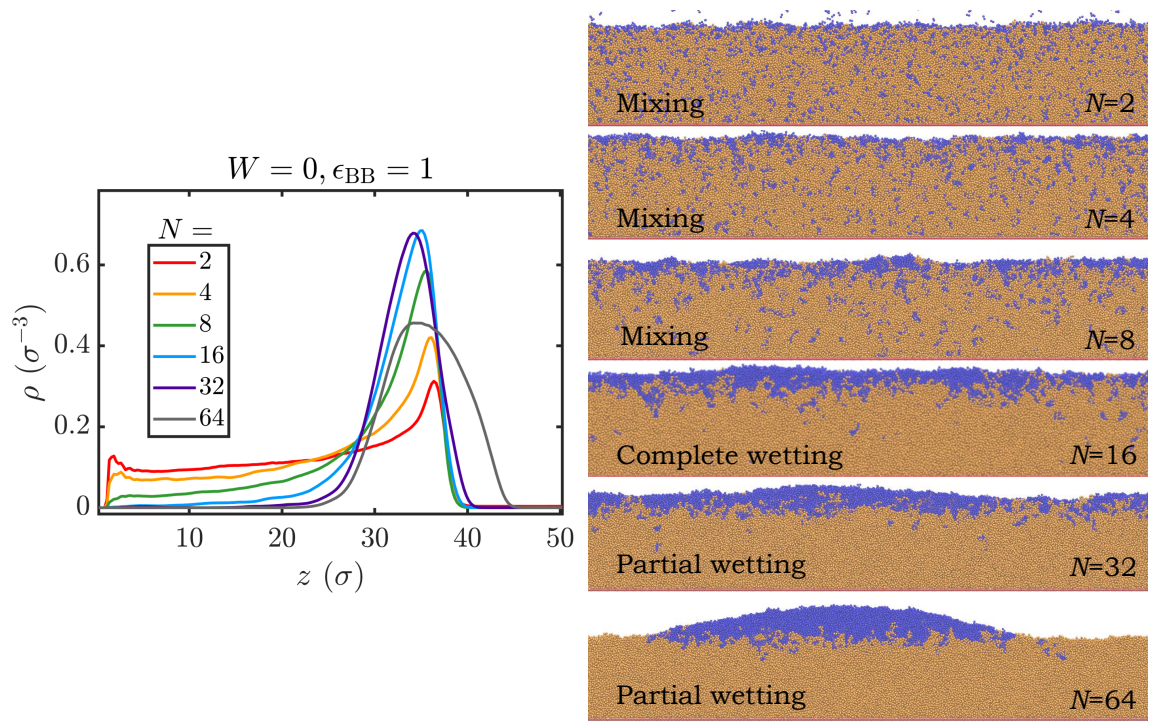

Figure 4.9: Density profiles for brush-melt systems at $\sigma_{\mathrm{GD}}=0.27, \epsilon_{\mathrm{BB}}=1.0$ and $W=0$, for variable $N$. Pictures next to the density profiles are snapshots of the brush-melt system for increasing $N$, illustrating the difficulty to clearly define a wetting state for systems that are close to a wetting transition.

locally, which we define as a droplet wetting the brush. Note that this subtlety only arises at the conjunction of the three phases; in general, the distinction between the wetting states is straightforward.

To quantify the effect of the degree of polymerization and the grafting densities on the brush wettability, figure 4.10 shows contact angle measurements $\theta$ versus $N$ and $\sigma_{\mathrm{GD}}$. The influence of $N$ on $\theta$ is given in the left and middle graphs, at $\epsilon_{\mathrm{BB}}=1.5$ and 1.0, respectively. Both graphs show a greater wettability for shorter polymer melt chains. This is a result of the increase in entropy for small chains, making the interfacial free energies $\gamma_{\mathrm{BL}}$ and $\gamma_{\mathrm{LV}}$ smaller, and thus the contact angle smaller. The different series were measured at specified values of $W$, showing that a higher interaction parameter results in a lower wettability. As a higher interaction parameter means that interactions between the brush and melt become less favorable, this behavior is as expected. The right graph of figure 4.10 shows the contact angle for varying grafting densities. The contact angles show a very weak dependence on the grafting density. The reason for this is that the last term in equation $\frac{4.8}{13}$ depends only weakly on the grafting density [47], namely $\Delta f_{\mathrm{ch}} \sigma_{\mathrm{GD}} \propto \sigma_{\mathrm{GD}}^{1 / 3}$. Since we do not vary the grafting density over several orders of magnitude, it is difficult to observe this dependency. 

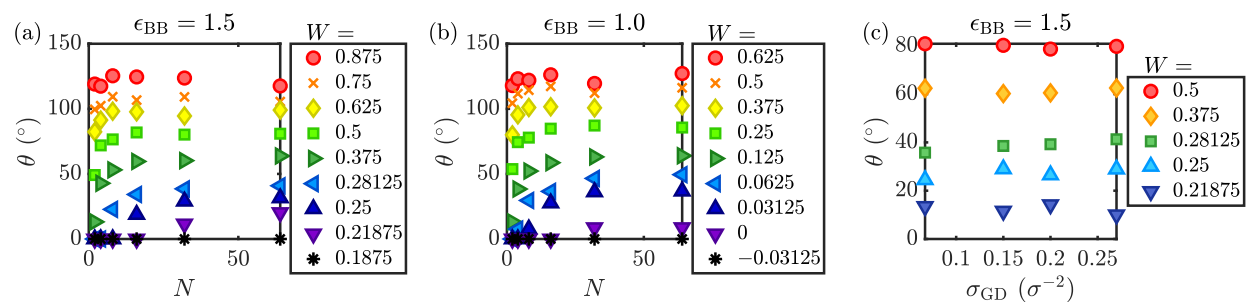

Figure 4.10: The contact angles plotted as a function of the polymer melt length $N(\mathrm{a}, \mathrm{b})$, and as a function of the grafting density $\sigma_{\mathrm{GD}}(\mathrm{c})$. Plotted are the contact angles for identical interaction parameters $W$. (a) and (b) were measured at $\sigma_{\mathrm{GD}}=0.15$, (c) was measured for $N=32$.

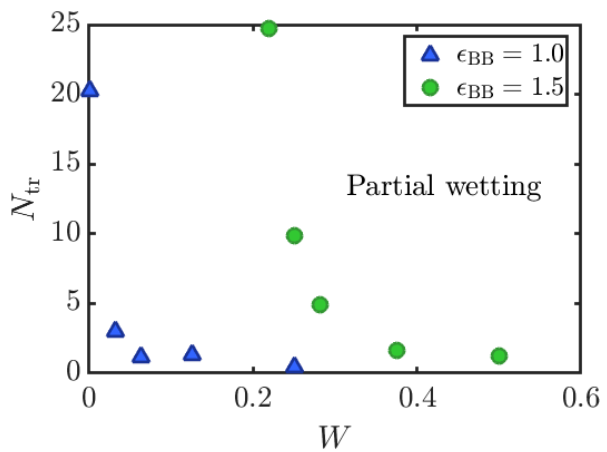

Figure 4.11: The transition point $N_{\text {tr }}$ from complete to partial wetting for varying interaction potentials $W$. The transition points are given for increased interactions at a constant $\epsilon_{\mathrm{BB}}$ of 1.0 and 1.5 in blue and green, respectively. Measurements were performed at $\sigma_{\mathrm{GD}}=0.15$

From figure 4.10 , the transition value $N_{\text {tr }}$ from complete to partial wetting can be obtained from the point where $\theta \rightarrow 0$. The resulting transition values $N_{\text {tr }}$ are shown in figure 4.11. This figure shows that $N_{\text {tr }}$ strongly decreases when $W$ is increased for both $\epsilon_{\mathrm{BB}}=1.0$ and $\epsilon_{\mathrm{BB}}=1.5$. The reason for this is that, shorter chains are less likely to partially wet, as smaller chains can gain more translational entropy by spreading than longer chains. Moreover, the data shows that there appears to be asymptotic values for $W$ at which $N_{\text {tr }}$ becomes very large. This result is consistent with other research [55], where high values of $N$ were found to reach an asymptotic value for the contact angle $\theta$ and thus the surface tension.

Next, we will consider the effect of the grafting density and melt polymer length on the interaction-based phase diagram of figure 4.1. To do so, we plot 
in figure 4.12 the contact angles as a function of the interaction parameter $W$ for the different melt-polymer lengths (left graph $\epsilon_{\mathrm{BB}}=1.5$ and middle graph $\epsilon_{\mathrm{BB}}=1.0$ ) and grafting densities (right graph). The data shows an increasing $\theta$ for increasing $W$, caused by the lower brush-melt interactions for higher interaction parameters, that directly influence the wettability between the brush and melt. The data also reveals an increased wettability for shorter melt chains, caused by the lower surface tensions of shorter polymer melt chains [56] (see also appendix 4.6.2, figure 4.19), due to their lower free energy as a result of the higher entropy, as compared to longer polymer melts. The
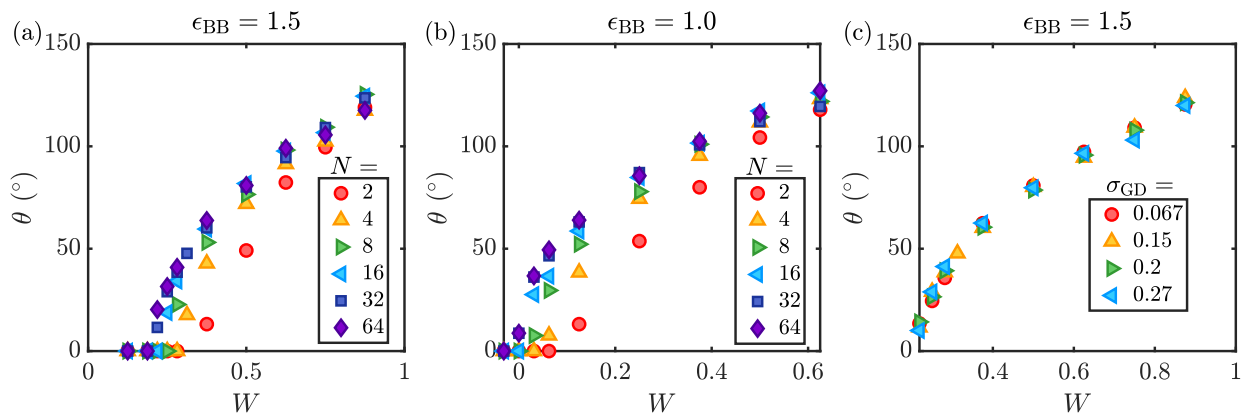

Figure 4.12: Contact angle measurements for varying degrees of the interaction parameter $W$. (a) Shows the contact angles $\theta$ for a brush wetted by a melt of varying degrees of polymerization $N$, at $\epsilon_{\mathrm{BB}}=1.5$ and $\sigma_{\mathrm{GD}}=0.15$. (b) Shows the contact angles of varying melt chain lengths at $\epsilon_{\mathrm{BB}}=1.0$ and $\sigma_{\mathrm{GD}}=0.15$. (c) Depicts the influence of the brush grafting density $\sigma_{\mathrm{GD}}$ on the contact angles for $N=32$.

right graph depicts $\theta$ plotted against $W$ for varying $\sigma_{\mathrm{GD}}$. This data illustrates again the rather weak dependency of the contact angle on the grafting density.

To examine how the complete to partial wetting transition in the interaction-based phase diagram (figure 4.1) changes upon varying the brush grafting density or melt chain length, we extract $W_{\text {tr }}$ from figure 4.12 by interpolating an exponential function fitted to the contact angles in figure 4.12. The resulting $W_{\mathrm{tr}}$ is shown in figure 4.13 , as a function of $N$ (left), and of $\sigma_{\mathrm{GD}}$ (right). The wetting transitions based on an enthalpic estimate are given as the blue and green dash-dotted lines, for $\epsilon_{\mathrm{BB}}=1.0$ and $\epsilon_{\mathrm{BB}}=1.5$, respectively. From the left graph, we see a rise in $W_{\text {tr }}$ to positive values for very short chains $N$. We can explain this through the increased wettability for small $N$, that was observed from figure 4.12. As mentioned before, short chains have lower surface tensions $\gamma_{\mathrm{BL}}$ and $\gamma_{\mathrm{LV}}$ (see also appendix 4.6.2, figure 4.19), owing to a higher entropic energy for shorter chains as compared to longer chains. For 
longer chains, we reach a constant value for $W_{\mathrm{tr}}$, as their surface tensions become similar (see figure 4.12). We assume this is caused by a limit to the intermolecular forces that can significantly contribute to the surface tension of a liquid melt. The right graph shows the dependency of $W_{\text {tr }}$ on the grafting density. As expected, we observe that $W_{\text {tr }}$ slightly decreases upon increasing the grafting density. As mentioned above, the reason for this is the rather weak dependency of the spreading parameter on the grafting density. This can also be understood from the steep decrease that is shown in the geometry-based phase diagram of figure 4.4, for $W>0.03125$ where mixing transitions occur at near-constant values of $N$ for all grafting densities. If we consider the asymptotic values for mixing in figure 4.4, we know that the mixing free energy must hardly vary for changing grafting densities. The data from figure 4.13(a) can

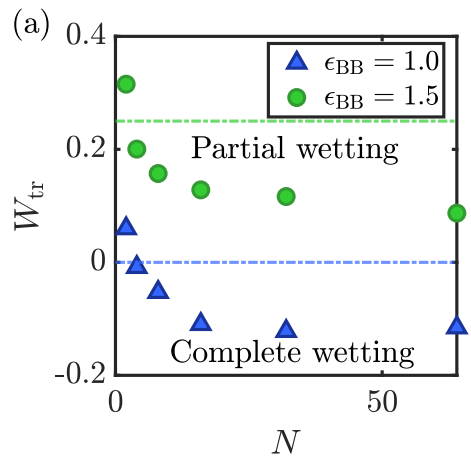

(b)

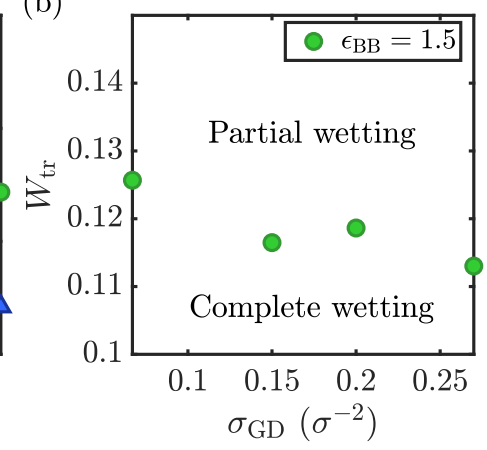

Figure 4.13: The interaction parameter transition points $W_{\mathrm{tr}}$, for complete to partial wetting, are plotted as (a) a function of the melt chain length $N$, and (b) the grafting density $\sigma_{\mathrm{GD}}$. The wetting transitions based on an enthalpic estimate are given as the blue and green dash-dotted lines, for $\epsilon_{\mathrm{BB}}=1.0$ and $\epsilon_{\mathrm{BB}}=1.5$, respectively.

be compared directly to the interaction-based phase diagram of figure 4.1. By doing so, we can compare how wetting transitions shift by changing the polymer melt length. From our data, we find that shorter $N$ values would cause the complete to partial wetting transition to shift to the right, compared to the interaction-based phase diagram of figure 4.1 (here, transitions are marked using black lines), which was measured for $N=32$. In fact, for the shortest melt chains, our $N_{\text {tr }}$ also shifts past the theoretical predictions as given in figure 4.1 (gray lines).

To conclude this paragraph, we suggest that a transition from complete to partial wetting should occur with respect to variation of geometric parameters $N$ and $\sigma_{\mathrm{GD}}$, even for settings at $W=0$, where we have not observed this to 
happen. Furthermore, we report greater wettability for shorter melt chains, due to their having a greater translational entropy gain for complete wetting, than their longer counterparts. We find no effects of the grafting density on the complete to partial wetting transitions.

\subsubsection{Partial wetting}
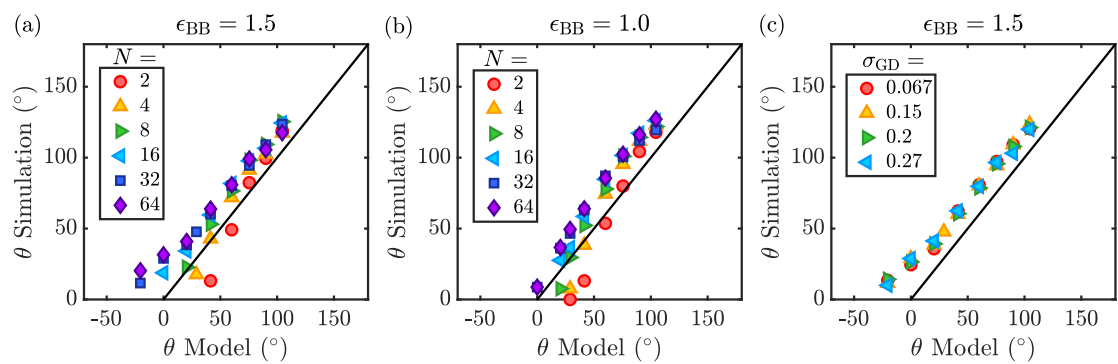

Figure 4.14: Contact angle measurements for different sets of measurements, plotted as a function of the enthalpic model prediction (equation 4.10). (a) Shows the contact angles for varying chain lengths of the melt $N$ measured at $\epsilon_{\mathrm{BB}}=1.5$ and $\sigma_{\mathrm{GD}}=0.15$. (b) Shows contact angles for varying chain lengths at $\epsilon_{\mathrm{BB}}=1.0$ and $\sigma_{\mathrm{GD}}=0.15$. (c) Shows contact angles for varying brush grafting densities $\sigma_{\mathrm{GD}}$ at $\epsilon_{\mathrm{BB}}=1.5$ and $N=32$.

Now that we have characterized the effect of entropy on the mixing to demixing transition and the complete to partial wetting transitions, we would now like to focus on entropic effects on the contact angles of the melts. We can make a theoretical prediction of the contact angles of the melt wetting the brush, according to an enthalpy-based interpretation of Young's law: [16]

$$
\cos (\theta)=\frac{2 \epsilon_{\mathrm{BL}}}{\epsilon_{\mathrm{LL}}}-1 .
$$

By plotting measured contact angles against the contact angles according to this enthalpic prediction in figure 4.14, we demonstrate deviations in our system from this purely enthalpic prediction. In the left and middle graph we varied the melt chain length, in the left graph for $\epsilon_{\mathrm{BB}}=1.5$, and for $\epsilon_{\mathrm{BB}}=1.0$ in the middle graph. The black line shows the enthalpic prediction. Overall, we find a reasonable agreement with the enthalpy-only model. However, many features are not captured. For example, we observe that short chains become increasingly hydrophilic, whereas long-chained melts show more hydrophobic behavior. This is in line with our results in figure 4.8 and figure 4.10, where shorter chains are more likely to mix or wet the brush completely, due to 
their lower surface tensions $\gamma_{\mathrm{BL}}$ and $\gamma_{\mathrm{LV}}$ shifting the spreading parameter $S$ to higher values. The right graph of figure 4.14 shows again that the contact angle depends only weakly on the grafting density. We can also see from all graphs in figure 4.14, that for small contact angles the effects of entropy are the largest. Furthermore, all cases show an offset between our enthalpic prediction and the actual contact angles, even for high contact angles. This offset cannot be caused by entropy and should, therefore, have a different origin.
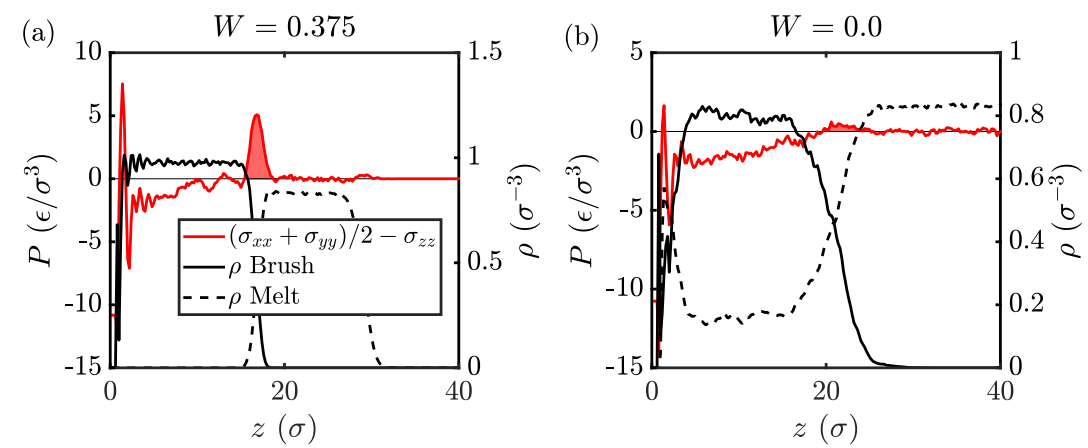

Figure 4.15: Two figures illustrating the challenge of measuring a surface tension close to the mixing to demixing transition. Shown are the total stress anisotropy $p=p_{\mathrm{T}}-p_{\mathrm{N}}$ (red), and the density profiles $\rho$ (black). The density profiles are divided in the brush profile (solid line) and the polymer melt (dashed line). The shaded area (red) in both graphs shows the surface tension peaks. (a) shows a system in the partial wetting regime $(W=0.375)$ and shows a clear surface tension peak. (b) shows the same system at the mixing regime $(W=0.0)$, where no clear surface tension peak is observed.

With the aim to obtain a more in-depth understanding of the deviations in the contact angle described above, we determine the effect of the melt chain length and grafting density on the interfacial tension $\Upsilon_{\mathrm{SL}}$ between the brush and the polymer melt using Kirkwood-Buff's analysis on the stress anisotropy. This is based on the relation:

$$
\Upsilon=\frac{1}{2} \int_{0}^{L_{z}}\left(p_{\mathrm{N}}(z)-p_{\mathrm{T}}(z)\right) d z,
$$

where $L_{z}$ is the system size in $z$, and $p_{\mathrm{N}}$ and $p_{\mathrm{T}}$ denote the pressure in the normal and tangential directions. However, as a brush does not have a constant density and no strictly defined interface, it is difficult to evaluate the interfacial tension involving a polymer brush. Here, we defined the interfacial tension of the brush according to Dimitrov's guidelines [57], meaning that we 
integrate over all positive values of the stress anisotropy away from the brushwall interface. In figure 4.15 we show the measured local stress and densities of the brush and melt phases, for two different values of $W$. The left graph is well into the partial wetting regime, and shows a clear surface tension peak (shaded red area). The right graph however, illustrates that it is challenging to define the brush-melt surface tension when entering the mixing regime, as this graph shows a mixed state at $\epsilon=1.0$ and $\sigma_{\mathrm{GD}}=0.15$ and $N=4$, where only a small peak can be observed. We expect mixing to only occur for values of $\Upsilon_{\mathrm{BL}}<0$, however. This positive $\Upsilon_{\mathrm{BL}}$ indicates again the difficulty in exactly defining the location of the wetting transitions, as discussed in section 4.2 in figure 4.9 .

Using Dimitrov's definition for the brush surface tension [57] $\Upsilon_{\mathrm{BL}}$, we plot the measured surface tensions in figure 4.16. Panels (a) and (b) show the surface tensions for varying melt chain lengths, respectively for $\epsilon_{\mathrm{BB}}=1.5$, and $\epsilon_{\mathrm{BB}}=1.0$. If we consider only enthalpic interactions, we expect $\Upsilon_{\mathrm{BL}} \sim W$,
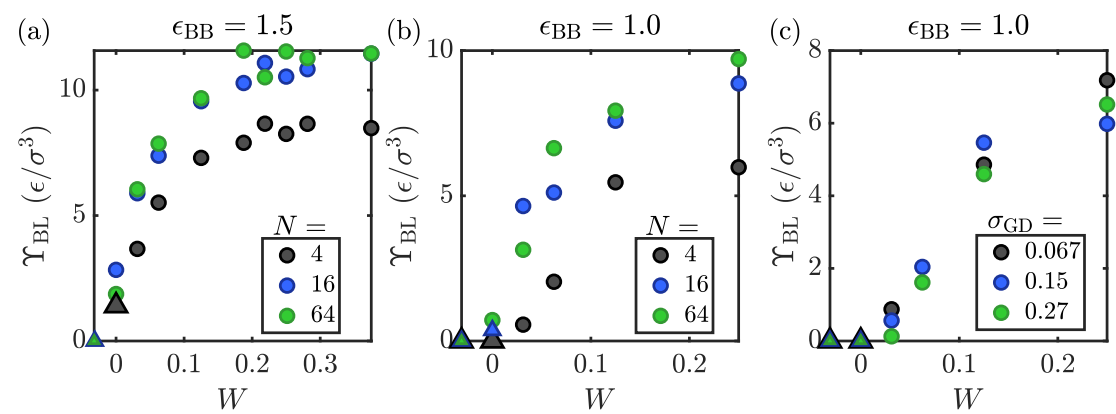

Figure 4.16: Interfacial tensions of brush-melt systems versus their interaction parameter $W$. (a) shows surface tensions at $\epsilon_{\mathrm{BB}}=1.5$ and $\sigma_{\mathrm{GD}}=0.15$, for a brush wetted by a melt of length $N=4$ (black), 16 (blue), and 64 (green). Circles denote an unmixed wetting state and triangles represent the mixing cases. (b) shows the surface tensions at $\epsilon_{\mathrm{BB}}=1$ and $\sigma_{\mathrm{GD}}=0.15$, for a brush wetted by a melt of the same lengths. (c) shows the surface tensions as measured at $\epsilon_{\mathrm{BB}}=1$ and $N=32$ for varying grafting densities $\sigma_{\mathrm{GD}}$, where the low grafting density is shown in black, the middle grafting density in blue, and the highest grafting density in green.

which we observe approximately, but we find a saturation for higher $W$. This saturation may be explained through the formation of a depletion layer between the brush and the melt phase, so that we measure an average interfacial tension of the brush-vacuum and the melt-vacuum, instead of a brush-melt interfacial tension. Furthermore, we expect smaller $N$ to give lower values of $\Upsilon_{\mathrm{BL}}$, as 
small chains have the capability to mix with brush chains, without causing the brush to lose much entropy due to stretching. From both of these graphs, we indeed observe that the surface tension is lower for smaller chains. We also observe a higher surface tension for a higher value of $\epsilon_{\mathrm{BB}}$, indicating that a collapsed brush has a higher surface tension than a more extended one, which is indeed what we find (see appendix 4.6.2).

In figure 4.16(c), we display the effect of the brush grafting density on the surface tension values of the brush-melt interface. From this data, we observe no significant effect of the grafting density on the surface tension within the statistical uncertainty. This is consistent with our other results where we did not observe effects of the grafting density on the wetting behavior of our brush, neither in the complete to partial wetting transition, nor in the contact angles. The cause of this observation is the same in all cases.

(a)

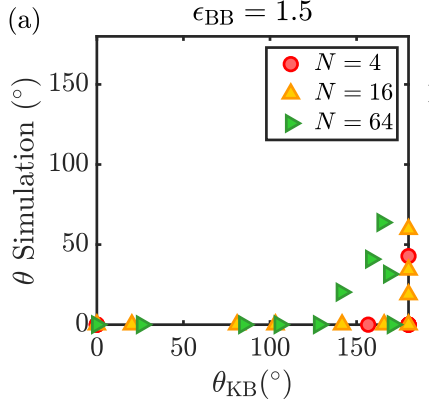

(b)

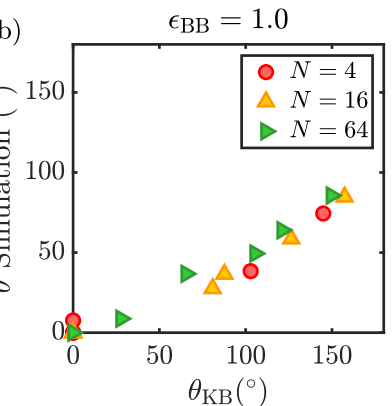

Figure 4.17: Contact angle measurements for different sets of measurements. (a) Shows the contact angles for varying chain lengths of the melt $N$ measured at $\epsilon_{\mathrm{BB}}$ 1.5. (b) Shows contact angles for varying chain lengths at $\epsilon_{\mathrm{BB}}$ 1.0. The contact angles given here, show the contact angle as measured from spherical fits through a droplet wetting a substrate, $\theta_{\theta}$, and the contact angle as determined from the surface tension measurements $\theta_{\gamma}$ Both graphs were measured at $\sigma_{\mathrm{GD}}=0.15$.

Lastly, we compare the contact angles extracted from our simulations to contact angles that we calculate via Young's law, using the surface tension extracted from our Kirkwood-Buff analysis (see also appendix 4.6.2 for values of $\Upsilon_{\mathrm{BV}}$ and $\Upsilon_{\mathrm{LV}}$ ). These results are summed up in figure 4.17. From this data, we see that the Kirkwood-Buff approach to predicting the contact angle is not very accurate; in particular given the comparatively good agreement with the enthalpic prediction shown in figure 4.14. This difference can be explained by considering that the contact angle measurement is based upon surface energies $\gamma$, and the Kirkwood-Buff analysis measures surface tensions 
$\Upsilon$ between the materials, which for complex elastic surfaces are very different quantities [58, 59].

\subsection{Conclusions}

In summary, we have shown that when considering the wetting transitions for a polymer brush wetted by a polymer melt, entropic effects can either aid or inhibit the transitions. For the mixing to demixing transition, enthalpic arguments predict the transition to occur at $W=0$, as the interaction potentials between all phases are equal at this point. We have shown that short chains and low grafting densities contribute a positive entropic effect to this transition, meaning that the transition from mixing to demixing is inhibited, as we observe mixing states for $W>0$. Long chains and high grafting densities contribute negatively, so that demixing is facilitated, for $W<0$. This is caused by the fact that polymer melts can win translational entropy by mixing, yet this translational entropy gain is smaller for longer polymer chains. Upon mixing, the polymer brushes pay an entropic penalty as they stretch out to allow for mixing. Our results for mixing and demixing compare well to predictions including both enthalpic and entropic effects, as developed by Aubouy et al 18 .

When considering the transition from complete to partial wetting, we find that short melt chains show higher wettability, meaning that they gain more translational entropy upon wetting a surface completely, as compared to long melt chains. As a result, the interfacial free energies for short chains, $\gamma_{\mathrm{BL}}$ and $\gamma_{\mathrm{LV}}$, are smaller. The effect of $N$ on the interaction parameter transition $W_{\mathrm{tr}}$ is quite strong for small $N$. This means that complete wetting is still very likely to occur for small $N$ even for very high melt-melt interactions. We observe that the complete to partial wetting transition depends only weakly on the grafting density, in agreement with predictions from de Gennes [47].

Lastly, we considered the partial wetting state, where we compared drop contact angles to measurements of surface tension $\Upsilon$. The comparison of our measured contact angles to an enthalpic prediction according to Young's law, shows good agreement, however entropic effects of the polymer chain length cause deviations in the exact behavior. Measurements of the surface tension $\Upsilon_{\mathrm{BL}}$ do not compare well to the measured drop contact angles, which we explain by considering that surface tensions as gathered from Kirkwood-Buff's analysis, are not equal to the surface energies $\gamma$ that are measured from contact angle measurements. In chapter 5, we will expand more upon this difference. 


\subsection{Appendix}

\subsubsection{Brush swelling}

Figure 4.18 shows the swelling ratio for a polymer brush, as a function of the brush-brush attraction $\epsilon / k T$. The inflection point indicates the point where an extended brush transitions into a collapsed brush.

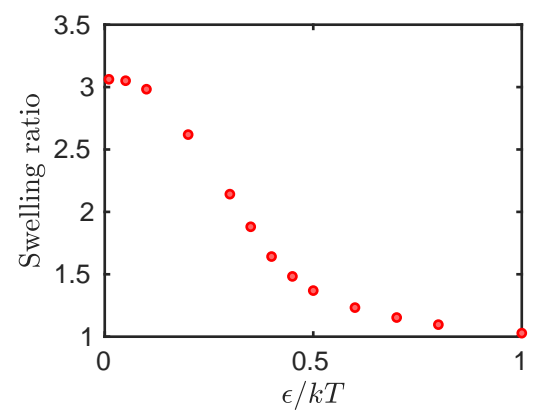

Figure 4.18: A diagram showing the swelling ratio of a polymer brush as a function of the brush-brush attraction $\epsilon / k T$. At the inflection point the brush transitions from an extended brush in good solvent to a collapsed brush in bad solvent.

\subsubsection{Surface tensions}

The table below shows the values of $\Upsilon_{\mathrm{BV}}$ for $\epsilon_{\mathrm{BB}}=1.0$ and $\epsilon_{\mathrm{BB}}=1.5$. The

\begin{tabular}{|c|c|}
\hline$\epsilon_{\mathrm{BB}}$ & $\Upsilon_{\mathrm{BV}}$ \\
\hline 1.0 & 2.70 \\
1.5 & 3.46 \\
\hline
\end{tabular}

values of $\Upsilon_{\mathrm{LV}}$ for varied chain lengths $N$ is shown in figure 4.19 


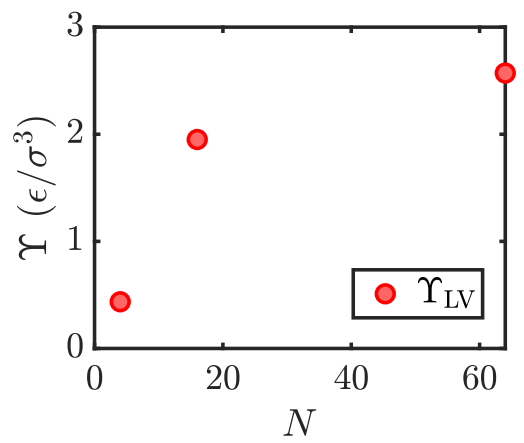

Figure 4.19: Shown are the surface tensions $\Upsilon_{\mathrm{LV}}$ for three increasing values of $N$, as measured according to the Kirkwood-Buff analysis.

\section{Bibliography}

[1] S. T. Milner, "Polymer brushes", Science 251, 905 (1991).

[2] O. Azzaroni, "Polymer brushes here, there, and everywhere: recent advances in their practical applications and emerging opportunities in multiple research fields", J. Polym. Sci. Part B: Polym. Chem. 50, 3225 (2012).

[3] W.-L. Chen, R. Cordero, H. Tran, and C. K. Ober, "50th anniversary perspective: Polymer brushes: Novel surfaces for future materials", Macromolecules 50, 4089-4113 (2017), URL https://doi.org/10.1021/acs. macromol.7b00450.

[4] I. Tokareva, S. Minko, J. H. Fendler, and E. Hutter, "Nanosensors based on responsive polymer brushes and gold nanoparticle enhanced transmission surface plasmon resonance spectroscopy", Journal of the American Chemical Society 126, 15950-15951 (2004), URL https://doi.org/10. 1021/ja044575y, pMID: 15584714.

[5] H. Merlitz, G.-L. He, C.-X. Wu, and J.-U. Sommer, "Nanoscale brushes: How to build a smart surface coating", Phys. Rev. Lett. 102, 115702 (2009), URL https://link.aps.org/doi/10.1103/PhysRevLett.102. 115702 .

[6] L. I. Klushin, A. M. Skvortsov, A. A. Polotsky, S. Qi, and F. Schmid, "Sharp and fast: Sensors and switches based on polymer brushes with adsorption-active minority chains", Phys. Rev. Lett. 113, 068303 (2014), URL https : //link .aps .org/doi/10.1103/PhysRevLett.113.068303. 
[7] M. Chen, W. H. Briscoe, S. P. Armes, and J. Klein, "Lubrication at physiological pressures by polyzwitterionic brushes.", Science 323, 1698-1701 (2009).

[8] J. Yu, N. E. Jackson, X. Xu, Y. Morgenstern, Y. Kaufman, M. Ruths, J. J. de Pablo, and M. Tirrell, "Multivalent counterions diminish the lubricity of polyelectrolyte brushes", Science 360, 1434-1438 (2018), URL http: //science.sciencemag.org/content/360/6396/1434

[9] Y. Yu, Y. Yao, S. van Lin, and S. de Beer, "Specific anion effects on the hydration and tribological properties of zwitterionic phosphorylcholinebased brushes", European Polymer Journal 112, 222-227 (2019).

[10] J. D. Willott, T. J. Murdoch, G. B. Webber, and E. J. Wanless, "Nature of the specific anion response of a hydrophobic weak polyelectrolyte brush revealed by afm force measurements", Macromolecules 49, 23272338 (2016), URL https://doi.org/10.1021/acs.macromol.5b02656.

[11] Y. Yu, R. A. Lopez de la Cruz, B. D. Kieviet, H. Gojzewski, A. Pons, G. J. Vancso, and S. de Beer, "Pick up move and release of nanoparticles utilizing co-non-solvency of pnipam brushes", Nanoscale 9, 1670-1675 (2017), URL http://dx.doi.org/10.1039/C6NR09245D.

[12] H. Yang, H. Zhu, M. M. R. M. Hendrix, N. J. H. G. M. Lousberg, G. de With, A. C. C. Esteves, and J. H. Xin, "Temperature-triggered collection and release of water from fogs by a sponge-like cotton fabric", Advanced Materials 25, 1150-1154 (2013), URL https://onlinelibrary. wiley.com/doi/abs/10.1002/adma.201204278.

[13] J. A. Howarter and J. P. Youngblood, "Self-cleaning and anti-fog surfaces via stimuli-responsive polymer brushes", Advanced Materials 19, 3838-3843 (2007), URL https://onlinelibrary.wiley.com/doi/abs/ 10.1002/adma.200700156.

[14] A. Halperin and P. G. de Gennes, "Wetting of polymer covered surfaces", Journal Physique 47, 1243-1247 (1986).

[15] D. Long, A. Ajdari, and L. Leibler, "How do grafted polymer layers alter the dynamics of wetting?", Langmuir 12, 1675-1680 (1996).

[16] L. I. S. Mensink, J. H. Snoeijer, and S. de Beer, "Wetting of polymer brushes by polymeric nanodroplets", Macromolecules 52, 2015-2020 (2019). 
[17] P. G. de Gennes, "Conformations of polymers attached to an interface", Macromolecules 13, 1069 (1980).

[18] M. Aubouy and E. Raphael, "Surface-tethered chains in polymeric matrixes", Journal de Physique II 3, 443-448 (1993).

[19] G. S. Grest, "Interfacial sliding of polymer brushes: a molecular dynamics simulation", Phys. Rev. Lett. 76, 4979 (1996).

[20] P. G. Ferreira, A. Ajdari, and L. Leibler, "Scaling law for entropic effects at interfaces between grafted layers and polymer melts", Macromolecules 31, 3994-4003 (1998), URL https://doi .org/10.1021/ma9712460.

[21] J. H. Maas, G. J. Fleer, F. A. M. Leermakers, and M. A. Cohen Stuart, "Wetting of a polymer brush by a chemically identical polymer melt: Phase diagram and film stability", Langmuir 18, 8871-8880 (2002).

[22] C. Pastorino, K. Binder, T. Kreer, and M. Müller, "Static and dynamic properties of the interface between a polymer brush and a melt of identical chains", The Journal of Chemical Physics 124, 064902 (2006), URL https://doi.org/10.1063/1.2162883.

[23] G. Reiter and R. Khanna, "Negative excess interfacial entropy between free and end-grafted chemically identical polymers", Phys. Rev. Lett. 85, 5599-5602 (2000), URL https://link.aps.org/doi/10.1103/ PhysRevLett.85.5599.

[24] X. Zhang, F. K. Lee, and O. K. C. Tsui, "Wettability of end-grafted polymer brush by chemically identical polymer films", Macromolecules 41, 8148-8151 (2008), URL https://doi.org/10.1021/ma801549r.

[25] M. Aubouy, G. H. Fredrickson, P. Pincus, and E. Raphael, "End-tethered chains in polymeric matrixes", Macromolecules 28, 2979-2981 (1995), URL https://doi.org/10.1021/ma00112a051.

[26] F. Zhou and W. T. S. Huck, "Surfaces grafted with polymer brushes as ideal building blocks for "smart" surfaces", Phys. Chem. Chem. Phys. 8, 3815 (2006).

[27] K. Park, S. H. Park, E. Kim, J.-D. Kim, S.-Y. An, H. S. Lim, H. H. Lee, D. H. Kim, D. Y. Ryu, D. R. Lee, and J. H. Cho, "Polymer brush as a facile dielectric surface treatment for high-performance, stable, soluble acene-based transistors", Chemistry of Materials 22, 5377-5382 (2010). 
[28] S. Liu, R. Eijkelenkamp, J. Duvigneau, and G. J. Vancso, "Silica-assisted nucleation of polymer foam cells with nanoscopic dimensions: Impact of particle size, line tension, and surface functionality", ACS Applied Materials \& Interfaces 9, 37929-37940 (2017).

[29] G. S. Grest and K. Kremer, "Molecular dynamics simulation for polymers in the presence of a heat bath", Phys. Rev. A 33, 3628 (1986).

[30] K. Kremer and G. S. Grest, "Dynamics of entangled linear polymer melts: A molecular-dynamics simulation", The Journal of Chemical Physics 92, 5057-5086 (1990), URL https://doi.org/10.1063/1.458541.

[31] D. Mukherji, C. M. Marques, and K. Kremer, "Collapse in two good solvents, swelling in two poor solvents: defying the laws of polymer solubility?", Journal of Physics: Condensed Matter 30, 024002 (2018), URL http://stacks.iop.org/0953-8984/30/i=2/a=024002.

[32] S. de Beer and M. H. Müser, "Friction in (im-) miscible polymer brush systems and the role of transverse polymer tilting", Macromolecules $\mathbf{4 7}$, 7666 (2014).

[33] S. de Beer, L. I. S. Mensink, and B. D. Kieviet, "Geometry-dependent insertion forces on particles in swollen polymer brushes", Macromolecules 49, 1070-1078 (2016).

[34] W. Humphrey, A. Dalke, and K. Schulten, "Vmd - visual molecular dynamics", J. Molec. Graphics 14, 33 (1996).

[35] G. S. Grest and M. Murat, "Structure of grafted polymeric brushes in solvents of varying quality: a molecular dynamics study", Macromolecules 26, 3108-3117 (1993), URL http://dx.doi.org/10.1021/ma00064a019

[36] J. H. Weijs, A. Marchand, B. Andreotti, D. Lohse, and J. H. Snoeijer, "Origin of line tension for a lennard-jones nanodroplet", Physics of Fluids 23, 022001 (2011), URL https://doi.org/10.1063/1.3546008.

[37] S. Plimpton, "Fast parallel algorithms for short-range molecular dynamics", Journal of Computational Physics 117, 1 (1995).

[38] T. Kreer, M. H. Müser, K. Binder, and J. Klein, "Frictional drag mechanisms between polymer-bearing surfaces", Langmuir 17, 7804 (2001).

[39] A. Milchev, D. I. Dimitrov, and K. Binder, "Polymer brushes with nanoinclusions under shear: A molecular dynamics investigation", Biomicrofluidics 4, 032202 (2010), URL http://scitation.aip.org/content/aip/ journal/bmf/4/3/10.1063/1.3396446. 
[40] M. E. R. Shanahan, "The influence of solid micro-deformation on contact angle equilibrium", Journal of Physics D: Applied Physics 20, 945 (1987), URL http://stacks .iop.org/0022-3727/20/i=7/a=018.

[41] E. R. Jerison, Y. Xu, L. A. Wilen, and E. R. Dufresne, "Deformation of an elastic substrate by a three-phase contact line", Phys. Rev. Lett. 106, 186103 (2011), URL https://link.aps.org/doi/10.1103/ PhysRevLett.106.186103.

[42] F. Léonforte and M. Müller, "Statics of polymer droplets on deformable surfaces", The Journal of Chemical Physics 135, 214703 (2011), URL https://doi.org/10.1063/1.3663381.

[43] J. N. Israelachvili, Intermolecular and Surface Forces (Academic Press, Elsevier, USA) (2011).

[44] R. Descas, J.-U. Sommer, and A. Blumen, "Grafted polymer chains interacting with substrates: computer simulations and scaling", Macromol. Theory Simul. 17, 429 (2008).

[45] L. G. MacDowell and M. Müller, "Adsorption of polymers on a brush: Tuning the order of the wetting phase transition", The Journal of Chemical Physics 124, 084907 (2006), URL https://doi.org/10.1063/1. 2172597

[46] E. Zhulina and O. Borisov, "Structure and stabilizing properties of grafted polymer layers in a polymer medium", Journal of Colloid and Interface Science 144, 507 - 520 (1991), URL http://www.sciencedirect.com/ science/article/pii/0021979791904166.

[47] P. G. de Gennes, "Wetting: statics and dynamics", Rev. Mod. Phys. 57, 827 (1985).

[48] L. Leibler, A. Ajdari, A. Mourran, G. Coulon, and D. Chatenay, "Wetting of grafted polymer surfaces by compatible chains", OUMS Conference on Ordering in Macromolecular Systems, Osaka 301 (1994).

[49] S. T. Milner, T. A. Witten, and M. E. Cates, "Theory of the grafted polymer brush", Macromolecules 21, 2610 (1988).

[50] A. N. Semenov, "Contribution to the theory of microphase layering in block-copolymer melts", Sov. Phys. JETP 61, 733-742 (1985).

[51] S. Alexander, "Adsorption of chain molecules with a polar head a scaling description", J. Phys.-Paris 38, 983 (1977). 
[52] C. Gay, "Wetting of a Polymer Brush by a Chemically Identical Polymer Melt", Macromolecules 30, 5939-5943 (1997), URL http://pubs.acs. org/doi/abs/10.1021/ma970107f

[53] M. W. Matsen and J. M. Gardiner, "Autophobic dewetting of homopolymer on a brush and entropic attraction between opposing brushes in a homopolymer matrix", The Journal of Chemical Physics 115, 2794-2804 (2001), URL https://doi.org/10.1063/1.1385557.

[54] I. Borukhov and L. Leibler, "Enthalpic stabilization of brush-coated particles in a polymer melt", Macromolecules 35, 5171-5182 (2002), URL https://doi.org/10.1021/ma011351g.

[55] B. Kim, D. Y. Ryu, V. Pryamitsyn, and V. Ganesan, "Dewetting of pmma on ps-brush substrates", Macromolecules 42, 7919-7923 (2009), URL https://doi.org/10.1021/ma9013498.

[56] D. N. Theodorou, "Variable-density model of polymer melt surfaces: structure and surface tension", Macromolecules 22, 4578-4589 (1989), URL https://doi .org/10.1021/ma00202a033.

[57] D. I. Dimitrov, A. Milchev, and K. Binder, "Polymer brushes in solvents of variable quality: Molecular dynamics simulations using explicit solvent", Journal of Chemical Physics 127 (2007).

[58] R. Shuttleworth, "The surface tension of solids", Proceedings of the Physical Society. Section A 63 (1950).

[59] B. Andreotti and J. H. Snoeijer, "Statics and dynamics of soft wetting", Annual Review of Fluid Mechanics (2020). 


\section{Chapter 5}

\section{Nanoscale elasto-capillary probes of the Shuttleworth effect ${ }^{*}$}

The surface energy of a solid interface can depend on the amount by which the surface is stretched. This strain-dependence gives rise to capillary phenomena that have no counterpart for simple liquid interfaces. Most notably one needs to carefully distinguish surface energy and surface tension, which is known as the Shuttleworth effect. However, the mechanical consequences of this effect, in particular for wetting, remain under debate. Here we explore the Shuttleworth effect in Molecular Dynamics simulations, comparing the outcomes of two independent tests on two model substrates (amorphous and crystalline, in the stiff regime) to direct measurements of the surface tensions. It is shown how this provides a fully consistent view on wetting in the presence of the Shuttleworth effect, and shows how elasto-capillarity emerges at the nanoscale. With this, we establish a framework that paves the way for systematic explorations of the Shuttleworth effect, for solid materials of increasing complexity.

\subsection{Introduction}

The interfacial mechanics of elastic substrates has recently gained much interest, since capillary forces are found to dramatically alter the mechanical response of soft solid matter [1-7]. The effect of surface tension that acts along the free surface of the solid has implications for technologies involving adhesion,

${ }^{*}$ To be submitted as: L.I.S. Mensink and J. H. Snoeijer, Nanoscale elasto-capillary probes of the Shuttleworth effect 
wetting, patterning and nanofabrication, and biomechanics [8, 9]. However, the proper description of these surface tension forces has remained controversial [10 16]. One of the reasons for this is that quantitative experiments probing surface rheology are challenging, as one needs to carefully separate surface effects from bulk elasticity. Another complication is conceptual: there is no consensus on how to properly interpret the mechanics of solid capillarity in the presence of three-phase contact lines.

The main conceptual difficulty is due to the fact that surface energy $\gamma$ of a solid substrate can depend on the amount of surface strain $\epsilon$. Such a strain-dependence $\gamma(\epsilon)$ has important implications that were first discussed by Shuttleworth in 1950 [17]. Most prominently this implies that the surface tension $\Upsilon$, i.e. the force per unit length pulling along the interface, has a different magnitude than the surface energy $\gamma$. This is a strong departure with respect to liquid interfaces, for which the interfacial tension is equal to the surface free energy. More precisely, the two quantities are related by the Shuttleworth equation [8, 9, 17, 18]

$$
\Upsilon=\gamma+(1+\epsilon) \frac{d \gamma}{d \epsilon},
$$

which for small strain reduces to the more familiar form $\Upsilon=\gamma+d \gamma / d \epsilon$. These concepts of surface tension and surface strain can be extended to a more precise tensorial form [8]. The Shuttleworth equation can be understood from the virtual work done by $\Upsilon$ to create new interfacial area by straining, leading to a change in surface free energy $d(\gamma A)=\gamma d A+A d \gamma$. The second term reflects a change of surface energy by straining, and is the origin of the term $d \gamma / d \epsilon$ in (5.1).

In the recent literature, a number of schemes have been proposed to experimentally probe the Shuttleworth effect. A conceptually straightforward approach [11] is based on Young's law for the liquid contact angle $\theta$, given by

$$
\gamma_{\mathrm{LV}} \cos \theta=\gamma_{\mathrm{SV}}-\gamma_{\mathrm{SL}},
$$

where $\gamma_{\mathrm{SV}}$ and $\gamma_{\mathrm{SL}}$ are the 'dry' and 'wet' surface energies of the solid. Upon straining the substrate and measuring the contact angle, one thus probes the strain-dependence of the difference $\gamma_{\mathrm{SV}}-\gamma_{\mathrm{SL}}$. A change in contact angle with $\epsilon$ was indeed observed experimentally, for a variety of drops on stiff glassy polymers [11. However, the contact angle remained constant on soft elastomeric surfaces. A second probe for of the Shuttleworth effect is based on an elastic version of the Wilhelmy plate [18 20]. Like the traditional Wilhelmy plate experiment, this consists of partially immersing a solid into a liquid reservoir, 
but now one measures the strain along the plate. In the presence of a Shuttleworth effect, one observes a discontinuity of elastic strain inside the solid that is proportional to the difference $\gamma_{\mathrm{SV}}^{\prime}-\gamma_{\mathrm{SL}}^{\prime}$; though the validity of this method was called into question [11. There are also proposals to directly determine the surface tension $\Upsilon$ of the solid interface. For very soft solid substrates, the surface is forced into a sharp corner and the contact angles follow from Neumann's law [10]. By measuring the contact angles as a function of strain, experiments have indeed probed a strain-dependent surface tension [10]. Also this method was called into question in a recent work [16]. Finally, Molecular Dynamics have offered a way to directly measure the surface tension as function of strain [12, 15], based on the Kirkwood-Buff method that is commonly used for liquid interfaces [21, 22]. Given the disparity of probes for the Shuttleworth effect, and the various concerns put forward in the literature, it is important to establish the validity of these methods by cross-checking their outcomes for a single system.

The aim of this paper is to provide a consistent interpretation framework for the Shuttleworth effect, by comparing the results of different tests discussed above. Using Molecular Dynamics simulations we perform three independent nano-scale probes of the Shuttleworth effect, namely stretching of the substrate, the elastic Wilhelmy plate, and direct measurement of the surface tension via Kirkwood-Buff. We consider two types of substrates, respectively consisting of a crystalline solid and an amorphous polymer gel. In both cases we focus on stiff regimes, for which there is no significant wetting ridge formed below the contact line (a finite wetting ridge would compromise a proper scale separation in the simulations). It is found that the three probes indeed give a consistent picture that is summarized in figure 5.1 the crystal exhibits a change in liquid contact angle upon straining, while this is not the case for the amorphous gel. This resembles experimental observations, and offers a clear mechanical interpretation of elastic wetting in the presence of the Shuttleworth effect.

\subsection{Molecular Dynamics simulations}

\subsubsection{Materials}

We performed Molecular Dynamics simulations of a generic liquid-vapor system, in contact with two different types of substrates. As a fluid we used a simple Lennard-Jones interaction potential between particles [23] 


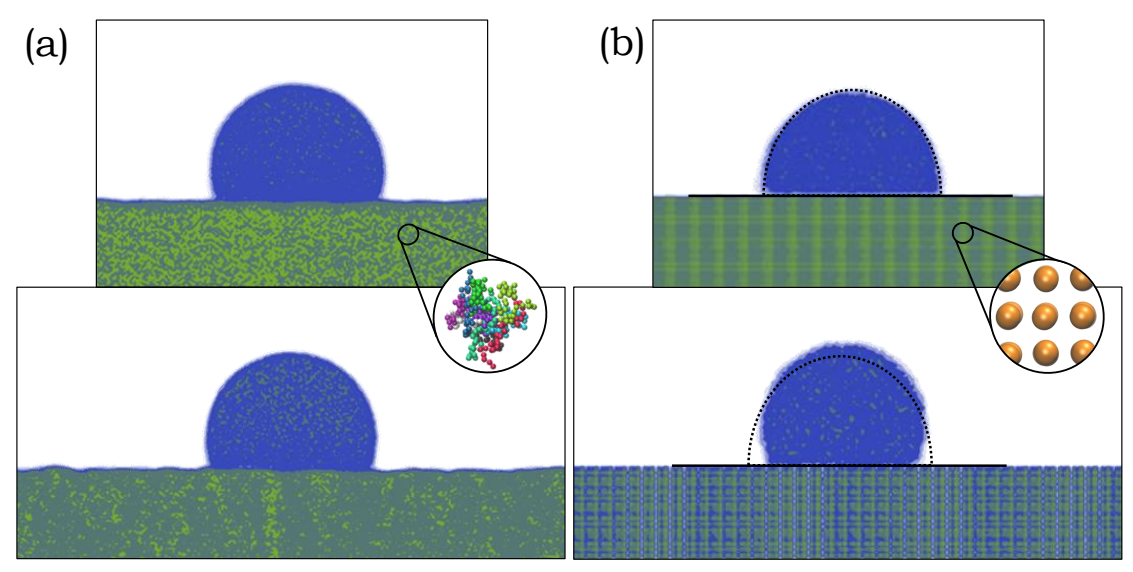

Figure 5.1: Wetting behavior of solids under external strain. The top panels and bottom panels respectively show a the wetting of a liquid drop before and after the substrate is stretched. (a) The substrate consists of a crosslinked polymer gel, for which the contact angle does not change upon straining. (b) The substrates consists of a Simple Cubic solid. To guide the eye, an isodensity contour of the unstrained droplet case is shown in the top and bottom figures of the crystal solid, showing the change in contact angle.

$$
U_{L J}\left(r_{i j}\right)=4 e_{\alpha \beta}\left[\left(\frac{d}{r_{i j}}\right)^{12}-\left(\frac{d}{r_{i j}}\right)^{6}\right],
$$

where $r_{i j}$ is the distance between two particles $i$ and $j, d$ is the distance between the repulsive core diameters of two particles, and $e_{\alpha \beta}$ is the interaction parameter between the particle species. Here we further introduced labels $\alpha$ and $\beta$ to denote the particle species (liquid L, or solid S), since all particles, not only the fluid, will interact via this Lennard-Jones potential. In our simulations, we used the potential with a cut-off at $2.5 d$.

For the substrates, we will compare results for a very stiff crystalline solid and a much softer amorphous gel that consists of a crosslinked polymer network. The crystalline solid is created using a simple mass and spring model of particles arranged into a simple cubic structure, similar to [20]. The particles are connected to their nearest neighbours and their next-nearest neighbours using simple harmonic springs. The lattice spacing between particles was taken at $a=0.8 d_{L L}$, while the spring constant was set at $k=385 e_{L L} / d_{L L}^{2}$. Below we will report calibrations of the Young's modulus for this system.

The second type of solid consists of a fully crosslinked polymer network that possesses an amorphous structure, both in the bulk and near the inter- 
face [4, 12, 15, 24]. This 'gel' was created by connecting $N=32$ Lennard-Jones particles into polymer chains, by adding a FENE (Finite Extensible Nonlinear Elastic) potential [25]:

$$
U_{\mathrm{FENE}}(r)=-\frac{1}{2} k_{s} R_{\text {max }}^{2} \ln \left(1-\frac{r^{2}}{R_{\max }^{2}}\right)+4 e\left(\frac{d}{r}\right)^{12} .
$$

For the FENE potential, we use a maximum extension $R_{\max }=1.5 d_{\mathrm{LL}}$ and a spring potential $k_{s}=25\left(\frac{e_{\mathrm{LL}}}{d_{\mathrm{LL}}^{2}}\right)$. These values allowed a sufficiently large timestep, without the occurrence of bond breaking [25]. A bath of these polymer chains was then equilibrated for $4.10^{6} \tau$ with a time step dt of $0.003 \tau$, where the timescale $\tau=\sqrt{\frac{m d^{2}}{e}}$, so their arrangement is amorphous. These polymer chains were connected into a fully crosslinked gel by randomly adding more FENE potentials between ('crosslinking') the polymeric chains. Crosslinks were also placed through the periodic boundary conditions, and no polymer chains were left uncrosslinked, so that one single, continuous gel-network is created. Self-interactions were avoided by making sure the smallest dimensions well exceed twice the radius of gyration. This gel has a finite elastic modulus, as will be characterized below. The stiffness can be varied by the degree of crosslinking [4, 12, 15, 24]. Here we deliberately focus on a comparatively stiff case, where during the wetting we do not observe the formation of a significant wetting ridge. This allows for a scale separation between the elasto-capillary length and the drop size, which is essential to ensure that Young's law can be safely applied [9].

All simulations were performed using GROMACS software [26 28]. Visualizations of the results were obtained using VMD (Visual Molecular Dynamics) [29]. Though we do not have a specific physical system in mind, we choose to report our results in SI units. For this, the mass of all particles was set to $m=10 \mathrm{amu}$, particle size to $d=0.34 \mathrm{~nm}$, and interaction potential to $e=3 k_{\mathrm{B}} T$ with the temperature $T=300 \mathrm{~K}$ in all cases. With this, the characteristic time of all systems is $\tau=\sqrt{\frac{m d^{2}}{e}}=0.6$ ps.

\subsubsection{Configurations}

The interface mechanics in the presence of the Shuttleworth effect will be explored systematically using three different setups, by which we obtain: (i) direct measurement of the surface tension and the elastic stress, (ii) measurement of the contact angle for stretched solids, (iii) strain-discontinuity using the 'elastic Wilhelmy plate' [18, 20]. Importantly, we will cross-check whether these different measurements give consistent mechanical interpretations of the Shuttleworth effect. 


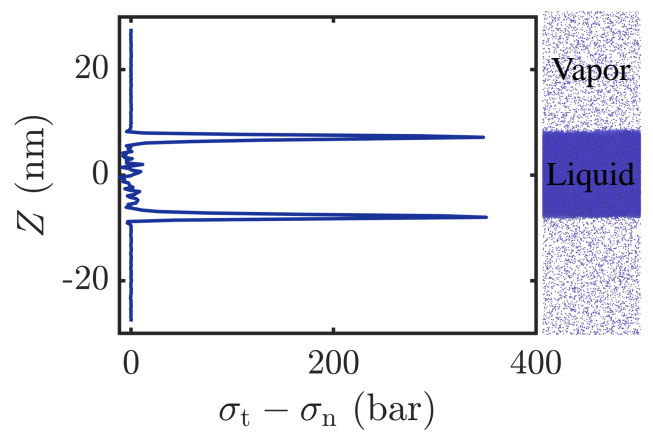

Figure 5.2: Stress anisotropy $\sigma_{\mathrm{t}}-\sigma_{\mathrm{n}}$ in the vicinity of a liquid-vapor interface of a Lennard-Jones fluids. The integral over the peak provides the liquid-vapor surface tension.

The first configuration consists of a slab of solid in contact with the LennardJones fluid, similar to the set-up for a fluid slab shown in figure 5.2. Surface tension $\Upsilon$ appears as a highly localized stress near the interface, which using the Kirkwood-Buff formula 21, 22] can be computed as:

$$
\Upsilon=\int d z\left(\sigma_{\mathrm{t}}-\sigma_{\mathrm{n}}\right),
$$

where the integral runs across the interface. Here, $\sigma_{\mathrm{t}}$ and $\sigma_{\mathrm{n}}$ respectively are the stress components tangential and normal to the interface. There are measured in the simulations using the GROMACS-LS version of GROMACS, as developed by J. M. Vanegas et al. [30, 31, which was designed specifically to serve this purpose for liquid interfaces. Here we apply the same method for solids. The Kirkwood-Buff measurements were performed on a system in an NVT ensemble, using a Nose-Hoover thermostat. Measurements were run for $20 \mathrm{~ns}$. In figure 5.2 we show the localized tangential stress near the vaporliquid interface, which in dimensional units gives a liquid-vapor surface tension $\Upsilon=\gamma_{\mathrm{LV}}=29 \mathrm{mN} / \mathrm{m}$. The surface tensions for elastic solids, and the subtleties associated to their accurate determination, will be discussed in Section 5.3

For the solid substrates, we also determined the elastic moduli by stretching the slab of bulk material in one direction (plain strain conditions), and measuring the bulk stress. The results are shown in figure 5.3, showing a linear relation between stress and strain for small deformations. In these plane-strain conditions, the slope give the elastic modulus $E=\tilde{E} /\left(1-\nu^{2}\right)$, where $E$ is the Young's modulus. The measured values respectively are $E=168 \mathrm{GPa}$ for the crystal, and $E=0.92 \mathrm{GPa}$ for the gel. This allows to evaluate the elastocapillary lengths $(\Upsilon / E)$ for both systems, respectively giving $\Upsilon / E=1.9 \cdot 10^{-4} \mathrm{~nm}$ for the very stiff crystal and $\Upsilon / E=0.07 \mathrm{~nm}$ for the gel. These lengths give 
the typical size of elastic displacements induced by a liquid drop. The stiffness of the gel can be varied by orders of magnitude upon reducing the density of crosslinks [4, 12, 15, 24. Here we deliberately consider a very stiff gel, so that the droplet does not induce any wetting ridge - in this case we can simply use Young's law to deduce information on the surface energies. For softer gels where a wetting ridge forms, the strict validity of Young's law is restricted [9] and for the present purpose is therefore less suitable.

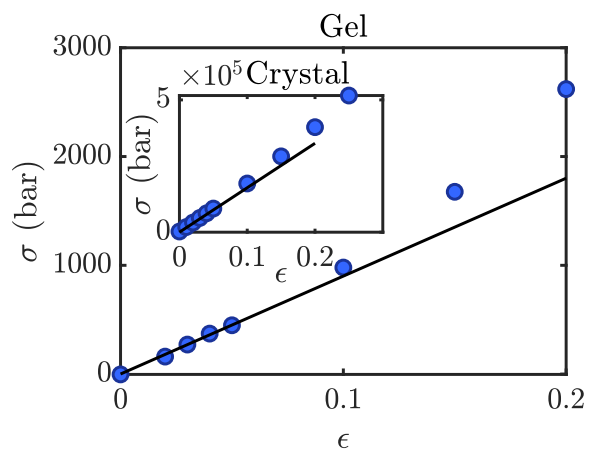

Figure 5.3: Determining the elastic modulus $E$ of the substrate by stressstrain curves. Main figure corresponds to the amorphous polymer gel $(E=$ $0.92 \mathrm{GPa})$, while the inset gives the result for the crystal $(E=168 \mathrm{GPa})$. The moduli are determined from the linear fits for small strain.

In the second setup, we simulated a thin quasi-two-dimensional slab of solid material that is in contact with a single droplet of Lennard-Jones fluid. Examples are provided in figure 5.1. In these measurements, we fixed the domain along the thin $y$-direction, to generate plane strain conditions, while allowing for an imposed strain $\epsilon$ in the $x$-direction. The simulations are carried out in an NPT ensemble, using the Nose-Hoover thermostat and ParrinelloRahman barostat. For the gel, the substrate consists of 430080 beads, with a droplet of 82680 particles. Dimensions prior to stretching are $63 \mathrm{~nm}$ and $100 \mathrm{~nm}$ along $x$ and $z$, while the thin $y$ direction was kept at $10 \mathrm{~nm}$. For the crystalline solid, the substrate and drop respectively consist of 262080 and 30750 particles. Dimensions prior to stretching are $63 \mathrm{~nm}, 36 \mathrm{~nm}$ along $x$ and $z$, and $7.5 \mathrm{~nm}$ along $y$. After equilibration for $10 \mathrm{~ns}$, measurements were averaged over $50 \mathrm{~ns}$ (or $10^{7}$ steps). From this system, contact angles of the drop wetting the substrates were measured from the droplets iso-density contours. Using the method described in [20], multiple contours are used to obtain an accurate fit of the droplet shape by a spherical cap. The contact angle follows from the intersection of this cap with the substrate, which is reported for various values of imposed strain $\epsilon$. 
For the third configuration, the elastic Wilhelmy plate, we used a thin, quasi-two-dimensional slab of solid that is partially immersed in a bath of Lennard-Jones fluid. A snapshot of this simulation is shown in figure 5.4 The top and the bottom of the plate are held fixed, but capillary effects near the contact line will generate elastic deformations inside the substrate. The simulation box is now periodic only in the $y$-direction, and in order to infer the strength of the Shuttleworth effect it is important that the plate has a large aspect ratio. Important parameters are thus the length of the plate $(55 \mathrm{~nm}$ for the crystal, $72 \mathrm{~nm}$ for the gel), and the plate width $(2.7 \mathrm{~nm}$ for the crytal, 6.7 for the gel). We simulated this set-up in an $N V T$ ensemble using the velocityrescaling thermostat. Measurements were equilibrated for $40 \mathrm{~ns}$. By comparing the particle position with and without the liquid, we infer the displacements induced by the presence of the contact line.

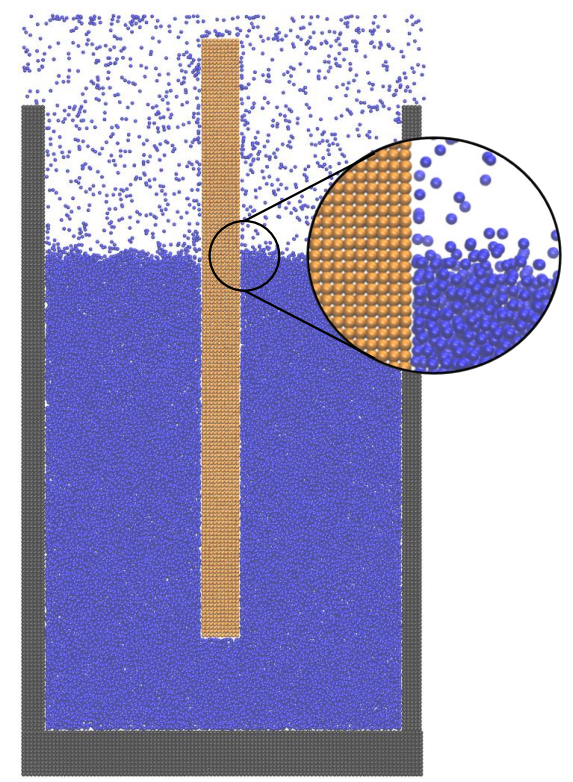

Figure 5.4: Simulation of the elastic Wilhelmy plate. The partially immersed plate is held fixed at the top and bottom. A discontinuity of strain across the contact line appears in the presence of the Shuttleworth effect. 


\subsection{Results}

\subsubsection{Contact angles}

The most direct manifestation of the Shuttleworth effect is via the liquid contact angle $\theta$, as illustrated in figure 5.1. As the solid is strained, a change in the contact angle implies a change of the difference $\gamma_{\mathrm{SV}}(\epsilon)-\gamma_{\mathrm{SL}}(\epsilon)$, via Young's law. The resulting contact angles are shown in figure 5.5, for both the crystalline solid and the amorphous gel. Clearly, the contact angle exhibits a strong dependence on the strain for the crystal (green triangles). The contact angle is observed to increase with strain, effectively rendering the solid more hydrophobic. For the gel, by contrast, $\theta$ exhibits no measurable dependence on the strain (blue circles). This either implies that there is no Shuttleworth effect, or that the difference $\gamma_{\mathrm{SV}}(\epsilon)-\gamma_{\mathrm{SL}}(\epsilon)$ remains constant. Note that both solids include data for negative $\epsilon$; we could not extend the simulations to stronger compressions, as these led to a buckling instability.

Let us remark that there is no sign of contact line pinning in our simulations. Namely, pinning would lead to an increase of the contact area of the drop when stretching and hence would lead to a reduction of $\theta$. Such a reduction is not observed for the gel nor for the crystal, ensuring that the measurement is not polluted by pinning - and that Young's law is indeed applicable.

The observations in figure 5.5 are perfectly in line with experimental measurements by Schulman et al. [11], who also observed no variation of the contact angle on incompressible elastomers, but a substantial effect on much stiffer glassy polymer substrates. However, the polymer substrates used here are much stiffer than the elastomers used in the experiment [11. This is important, since the condition of 'equality of chemical potential' [9, 13], possibly leading to $\gamma_{\mathrm{SL}}^{\prime} \approx \gamma_{\mathrm{SV}}^{\prime}$, no longer applies in our simulations. Hence, there is no thermodynamic reason why the system would adapt to achieve $\gamma_{\mathrm{SL}}^{\prime} \approx \gamma_{\mathrm{SV}}^{\prime}$. So it is likely that the derivatives $\gamma_{\mathrm{SV}}^{\prime}$ and $\gamma_{\mathrm{SL}}^{\prime}$ are both equal to zero (though we cannot exclude that they are nonzero and happen to cancel out).

To further quantify the Shuttleworth effect for the crystal, we expand Young's law 5.1 in the limit of small strain,

$$
\cos \theta_{\mathrm{Y}}=\cos \theta_{0}+\varepsilon\left[\gamma_{\mathrm{SV}}^{\prime}(0)-\gamma_{\mathrm{SL}}^{\prime}(0)\right] / \gamma,
$$

where $\theta_{0}$ is the contact angle at vanishing strain. From this expansion it is clear that the slope of the data in figure 5.5 provides the strength of $\gamma_{\mathrm{V}}^{\prime}-$ $\gamma_{\mathrm{SL}}^{\prime}$. The green dashed line through the data gives the Shuttleworth effect, in terms of the difference $\gamma_{\mathrm{SV}}^{\prime}-\gamma_{\mathrm{SL}}^{\prime}=-0.95 \gamma_{\mathrm{LV}}$ for the crystal (at $\epsilon \approx 0$ ). This value is actually not fitted, but obtained independently from the elastic 


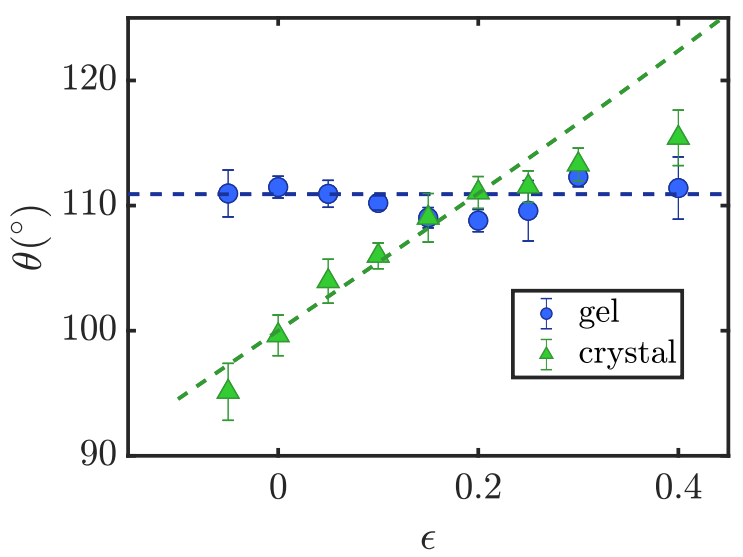

Figure 5.5: Contact angle $\theta$ for substrates placed under various strain $\epsilon$. The blue circles represent contact angle measurements of the amorphous polymer gel substrate. The black triangles represent the crystal substrate. The green dashed line corresponds to $\gamma_{\mathrm{SV}}^{\prime}-\gamma_{\mathrm{SL}}^{\prime}=-0.95 \gamma_{\mathrm{LV}}$, as obtained from the elastic Wilhelmy plate measurement on the crystal. The blue dashed line indicates that we could not measure a systematic change of $\theta$ with $\epsilon$.

Wilhelmy plate experiment that will be discussed below. Indeed, it provides a perfectly consistent description of our contact angle measurements. Hence, the derivative with respect to strain is comparable to the typical scale of the surface energies, and the Shuttleworth effect is an order unity contribution to the surface tension. The horizontal blue dashed line indicates there is no measurable dependence for the gel.

For later reference, we also report the contact angles at $\epsilon=0$. For the crystal we obtain $\theta_{0}=100^{\circ}$, while for the gel $\theta_{0}=112^{\circ}$. From this one can infer the difference in surface energies at $\epsilon=0$, as respectively $\gamma_{\mathrm{SV}}-\gamma_{\mathrm{SL}}=-0.17 \gamma_{\mathrm{LV}}$ for the crystal, and $\gamma_{\mathrm{SV}}-\gamma_{\mathrm{SL}}=-0.37 \gamma_{\mathrm{LV}}$ for the gel.

\subsubsection{Elastic Wilhelmy plate}

We now explore the second method to probe the Shuttleworth effect, which is based on the elastic Wilhelmy plate (cf. figure 5.4). Owing to the slenderness of the plate, the vertical strain away from the contact line will be homogeneous inside the solid. However, in the presence of a Shuttleworth effect, a discontinuity in strain will appear across the contact line. This is captured by

$$
\epsilon^{+}-\epsilon^{-}=\frac{2\left(\gamma_{\mathrm{SL}}^{\prime}-\gamma_{\mathrm{SV}}^{\prime}\right)}{E W}
$$


where $W$ is the width of the plate, $\epsilon=d u_{z} / d z$ is the strain based on the vertical displacement, while the + and - respectively indicate the dry and immersed parts of the solid. This discontinuity can be understood by drawing a control volume over a region that includes the contact line zone, and by computing the resultant capillary forces that are exerted onto the system. First, the liquid-vapour interface pulls with a vertical downward component $\gamma \cos \theta=\gamma_{\mathrm{SV}}-\gamma_{\mathrm{SL}}$. The solid surface tensions pull upwards with $\Upsilon_{\mathrm{SV}}-\Upsilon_{\mathrm{SL}}$. Combined with the liquid-vapor force, using also (5.1), this gives rise to an upward resultant capillary force $\gamma_{\mathrm{SV}}^{\prime}-\gamma_{\mathrm{SL}}^{\prime}$, on both sides of the plate. To achieve equilibrium, this must be balanced by a difference in elastic tension, $W E \epsilon^{+}-W E \epsilon^{-}$, leading to 5.7 ).

In the simulations we measured the displacement of particles from such a plate that is partially immersed inside of a liquid bath, as compared to that same plate kept suspended in a gas environment. Figure 5.6.(a) shows the displacements $u_{z}$ for the crystalline solid. The bottom and top of the plate are kept fixed $\left(u_{z}=0\right)$, while the contact line is located at $z=0$. Clearly, there is a difference in strain $\epsilon$ between the wetted part of the plate $(z<0)$ and the dry part $(z>0)$. The immersed zone has a negative strain, $\epsilon^{-}=-4.5 \cdot 10^{-5}$, and is therefore compressed. The upper part has a positive strain, $\epsilon^{+}=7.6 \cdot 10^{-5}$, and is stretched. This observed strain-discontinuity is in line with previous simulations [20] and experiment [19], signalling the presence of the Shuttleworth effect.

We are now in a position to quantify the strength of the Shuttleworth effect, inserting the observed strain discontinuity in (5.7). Given that $W=2.7 \mathrm{~nm}$, and $E=168 \mathrm{GPa}$, we obtain

$$
\gamma_{\mathrm{SV}}^{\prime}-\gamma_{\mathrm{SL}}^{\prime}=-0.95 \gamma_{\mathrm{LV}}
$$

So even though the strains are extremely small in the simulations, owing to the large value of the Young modulus, the Wilhelmy plate can be used to demonstrate a strong Shuttleworth effect. The measured value can be translated to a change in contact angle with strain, which has been superimposed as the green dashed line in figure 5.5. It indeed provides a very accurate description of the observed change of the liquid angle. Hence, the two independent probes of the Shuttleworth effect, contact angle measurement and the elastic Wilhelmy plate, provide a consistent measurement of solid capillarity near the contact line.

We now turn to the elastic Wilhelmy plate measurement on the amorphous gel. Given the absence of a strain-dependence on the contact angle in figure 5.5, we do not expect a measurable discontinuity of strain for the gel. The displacements are shown in figure 5.6(b), where we zoom in on the noisy signal 

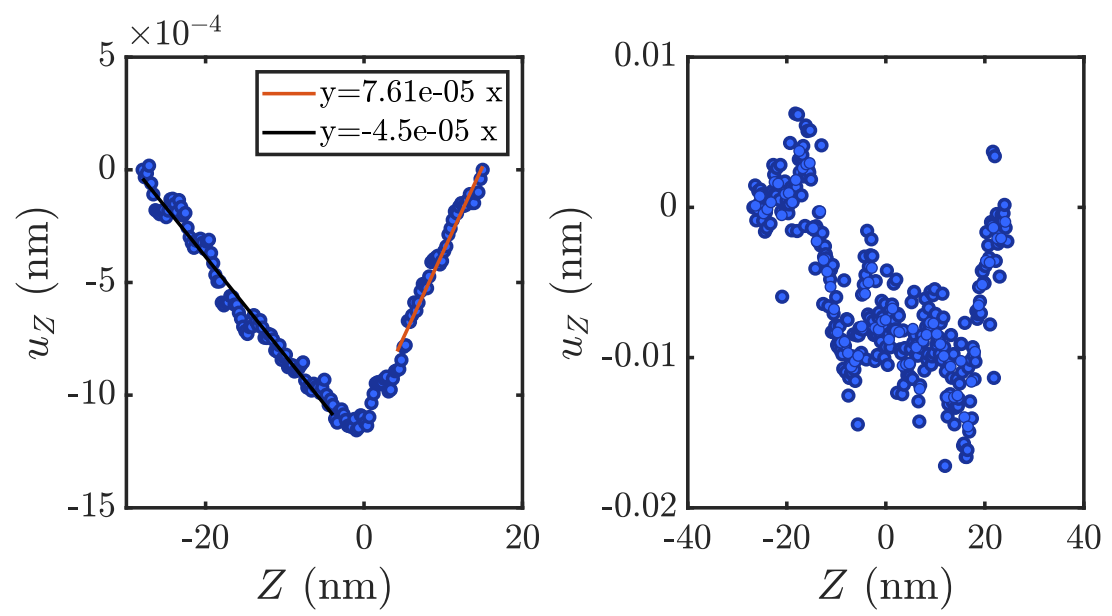

Figure 5.6: Elastic displacement in the vertical direction $u_{z}$ along the elastic Wilhelmy plate. The top and bottom of the plate are held fixed $\left(u_{z}=0\right)$, while the contact line is located at $z=0$. The left figure shows displacement for a crystal wire, for which a clear discontinuity in strain $d u_{z} / d z$ is observed. The right figure provides a zoom on the noisy signal obtained for the amorphous gel (see text).

that we could measure. Given the scatter of data we refrain from trying to impose linear fits, but we can still use the signal to obtain an upper bound for the Shuttleworth effect. Given that the maximum displacements are of the order of $0.01 \mathrm{~nm}$, spread out over $20 \mathrm{~nm}$ along the plate, we estimate an upper bound for $\left|\epsilon^{+}-\epsilon^{-}\right|<0.5 \cdot 10^{-3}$. For a plate of $W=6.7 \mathrm{~nm}$ and $G=0.92 \mathrm{GPa}$, this implies that for the amorphous gel

$$
\left|\gamma_{\mathrm{SV}}^{\prime}-\gamma_{\mathrm{SL}}^{\prime}\right|<0.05 \gamma_{\mathrm{LV}}
$$

Hence, based on the Wilhelmy plate measurement, one concludes that the Shuttleworth effect would at most contribute by the order of a few percent. This is again consistent with the contact angles in figure 5.5. which exhibit no measurable dependence with imposed strain.

\subsubsection{Direct measurement of surface tension}

Molecular dynamics simulations in principle allow for a direct measurement of the surface tension, using the stress-anisotropy near the interface. For this one can make use of the Kirkwood-Buff relation (5.5), usually applied to liquidliquid interfaces. Indeed, a similar localized stress-anisotropy appears for elas- 
tic substrates [15], and we now investigate this in our simulations. The result is shown in figure 5.7, where we plot $\sigma_{\mathrm{t}}-\sigma_{\mathrm{n}}$ across the interface. The integral over each of the peaks provides the surface tension that is pulling along the interface.
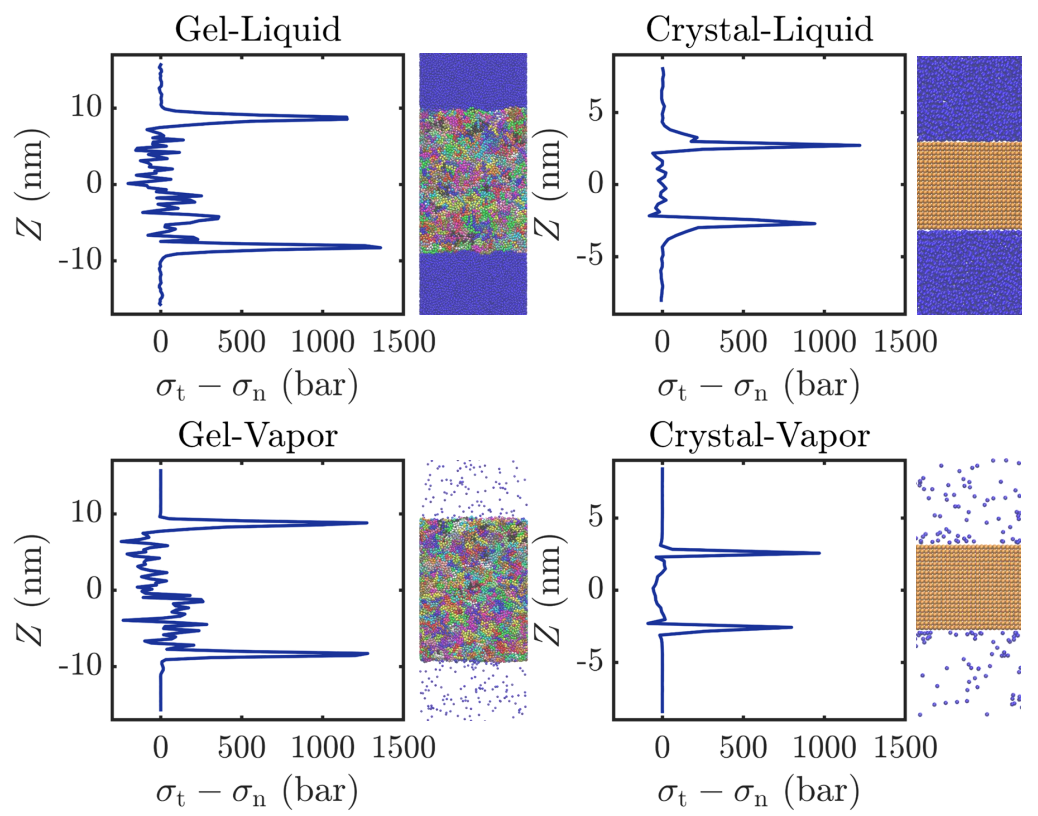

Figure 5.7: Direct measurement of surface tension $\Upsilon$ using the Kirkwood-Buff relation, by integrating over the stress anisotropy $\sigma_{\mathrm{t}}-\sigma_{\mathrm{n}}$ at the interface. The top and bottom panels respectively correspond to solid-liquid and liquid-vapor interfaces, for the amorphous gel (left) and the crystal (right).

In practice, however, elastic substrates pose an important difficulty that is not present for liquids. Namely, the elasticity will induce stress-anisotropy in the bulk of the material, which needs to be separated from the anisotropy in the interface. To illustrate this we compare the stress profiles inside a gel substrate, obtained for a case without stretching $(\epsilon=0)$ and with stretching $(\epsilon=0.15)$. Figure 5.8 (a) shows that the peaks corresponding to the interfaces are slightly closer for the stretched case (blue data) as compared to the unstretched case (black data). This is a consequence of the incompressibility of the medium; the stretching along the substrate leads to a reduction of the substrate thickness. This effect can also be seen directly from the density profiles in figure 5.8(b).

The most important feature in figure 5.8(a) is that a nonzero signal appears in between the two peaks for the stretched case (blue). This is the bulk elastic stress, which arises due to the finite elastic modulus. Importantly, to measure 

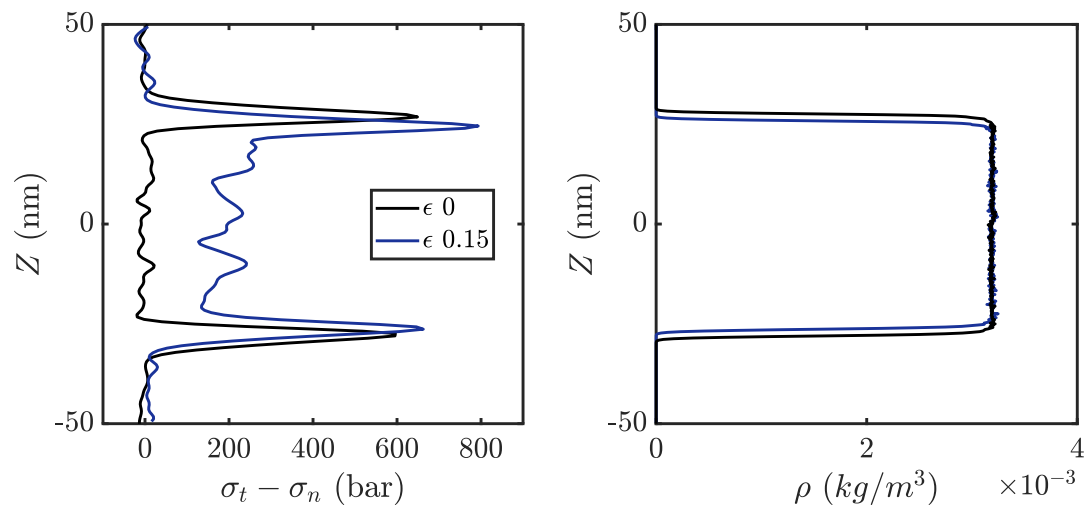

Figure 5.8: Left: stress anisotropy $\sigma_{\mathrm{t}}-\sigma_{\mathrm{n}}$ for the solid-vapor interface of the amorphous gel, with and without strain. Right: Corresponding density profiles. The black line corresponds to $\epsilon=0$, the blue line to $\epsilon=0.15$.

the surface tension one needs to decide which part of the signal belongs to the surface tension, and which part is purely bulk elasticity - hence we need to subtract the bulk signal. This is not a problem as long as typical values of the stress inside the interface $\gamma / a$ (where $a$ is the width of the interface) are large compared to the elastic stress $\sim E \epsilon$, but for a case such as in figure 5.8(a) it is not clear how this can be done unambiguously.

In order to obtain well-defined values of the surface tension, we adapt the size of the simulation domain such that the stress anisotropy is in bulk is minimal (figure 5.7). For the amorphous gel, this gives $\Upsilon_{\mathrm{SL}}=98 \mathrm{mN} / \mathrm{m}$, and $\Upsilon_{\mathrm{SV}}=83 \mathrm{mN} / \mathrm{m}$. Given that in the previous sections we concluded that $\gamma_{\mathrm{SV}}^{\prime}-\gamma_{\mathrm{SL}}^{\prime} \approx 0$, the Kirkwood-Buff values gives a direct prediction for the surface energies, i.e. $\gamma_{\mathrm{SV}}-\gamma_{\mathrm{SL}}=-0.52 \gamma_{\mathrm{LV}}$. Applying Young's law, this corresponds to a contact angle $\theta_{0}=121^{\circ}$, which is in reasonable agreement with the observed contact angle $110^{\circ}$. To probe the uncertainty in the KirkwoodBuff measurement associated to elastic stress, we verified that a change in box-size of $\pm 1 \%$ gives variations in $\Upsilon_{\mathrm{SL}}$ of about $\pm 10 \mathrm{mN} / \mathrm{m}$. Such a variation could be the source of the difference with the observed contact angle.

Quantitative measurements for the surface tension are even more challenging for the much stiffer, crystalline solid, as elastic effects are even more difficult to remove. The values obtained in our simulations are $\Upsilon_{\mathrm{SL}}=42 \mathrm{mN} / \mathrm{m}=$ $1.46 \gamma_{\mathrm{LV}}$, and $\Upsilon_{\mathrm{SV}}=25 \mathrm{mN} / \mathrm{m}=0.87 \gamma_{\mathrm{LV}}$ for the crystal. To estimate the uncertainty of these values, we compare them to the measurement of $\theta_{0}$, which gave $\gamma_{\mathrm{SV}}-\gamma_{\mathrm{SL}}=-0.17 \gamma_{\mathrm{LV}}$, combined with that of the elastic Wilhelmy plate, for which $\gamma_{\mathrm{SV}}^{\prime}-\gamma_{\mathrm{SL}}^{\prime}=-0.95 \gamma_{\mathrm{LV}}$. Applying the Shuttleworth equation (5.1), this gives $\Upsilon_{\mathrm{SV}}-\Upsilon_{\mathrm{SL}}=-1.12 \gamma_{\mathrm{LV}}$, which is approximately a factor 2 larger than 
the value predicted by the Kirkwood-Buff measurement. This emphasizes once more the challenge of directly measuring the surface tension in the presence of strong elastic effects.

\subsection{Discussion}

To summarize, we have systematically explored various probes of the Shuttleworth effect as proposed in the recent literature, applied at the nanoscale using Molecular Dynamics simulations. We have compared the results for two different model substrates, consisting of a crystalline solid and an amorphous polymer gel. While the gel is soft compared to the crystal, it is still sufficiently stiff so that the elastocapillary length $\gamma / E$ is small compared to molecular scales so that no significant wetting ridge is formed.

It is found that the contact angle of a wetting drop changes significantly when straining the crystalline substrate, while no effect was observed for the gel - providing direct evidence of a strong Shuttleworth effect only for the crystal. This is in good agreement with recent experimental observations [11]. This observed strain-dependence is in quantitative agreement with the measurement of $\gamma_{\mathrm{SV}}^{\prime}-\gamma_{\mathrm{SL}}^{\prime}$ from the elastic Wilhelmy plate, providing a clear validation of these two probes of the Shuttleworth effect. A direct measurement of the surface tension using the Kirkwood-Buff relation turned out more challenging; the main reason being that it is difficult to separate the interface contribution from bulk elastic stress anisotropy. While qualitatively consistent with the other probes, the actual values appear to differ up to a factor 2 for the stiff solid. An accurate measurement of surface tension as function of imposed strain using the Kirkwood-Buff relation might be feasible for very soft materials, such as done in [12, 15]; however, care needs to be taken that the values do not depend on how the bulk elastic stress is removed when performing the integrals.

The results presented here provide a clear mechanical interpretation of how wetting is altered by the Shuttleworth effect, for the case of comparatively stiff solids. While this paves the way for a systematic exploration for more complex substrates, it remains to be explored how the Shuttleworth effect depends on the molecular structure of more complex, exceedingly soft materials.

\section{Bibliography}

[1] S. Mora, T. Phou, J.-M. Fromental, L. M. Pismen, and Y. Pomeau, "Capillarity driven instability of a soft solid", Phys Rev Lett 105, 214301 (2010).

[2] A. Marchand, S. Das, J. H. Snoeijer, and B. Andreotti, "Contact angle 
on a soft solid: From young's law to neumann's law", Physical Review Letters 109 (2012).

[3] R. W. Style and E. R. Dufresne, "Static wetting on deformable surfaces, from liquids to soft solids", Soft Matter 8, 7177-7184 (2012).

[4] Z. Cao, M. J. Stevens, and A. V. Dobrynin, "Elastocapillarity: Adhesion and wetting in soft polymeric systems", Macromolecules 47, 6515-6521 (2014), URL https://doi.org/10.1021/ma5013978.

[5] C.-Y. Hui, T. Liu, T. Salez, E. Raphael, and A. Jagota, "Indentation of a rigid sphere into an elastic substrate with surface tension and adhesion", Proc. R. Soc. A 471, 20140727 (2015).

[6] N. Naderman, C.-Y. Hui, and A. Jagota, “?”, PNAS 110, 10541 (2013).

[7] S. Mondal, M. Phukan, and A. Ghatak, "?", PNAS 112, 12565 (2015).

[8] R. W. Style, A. Jagota, C.-Y. Hui, and E. R. Dufresne, "Elastocapillarity: Surface tension and the mechanics of soft solids", Annual Review of Condensed Matter Physics 8, 99-118 (2017), URL https://doi .org/10. 1146/annurev-conmatphys-031016-025326.

[9] B. Andreotti and J. H. Snoeijer, "Statics and dynamics of soft wetting", Annual Review of Fluid Mechanics (2020).

[10] Q. Xu, K. Jensen, R. Boltyanskiy, R. Sarfat, R. W. Style, and E. R. Dufresne, "Direct measurement of strain-dependent solid surface stress", Nature Communications 8, 555 (2017).

[11] R. D. Schulman, M. Trejo, T. Salez, E. Raphael, and K. Dalnoki-Veress, "Surface energy of strained amorphous solids", Nature Communications 9, 982 (2018).

[12] H. Liang, Z. Cao, Z. Wang, and A. V. Dobrynin, "Surface stresses and a force balance at a contact line", Langmuir 34, 7497-7502 (2018), URL https://doi.org/10.1021/acs.langmuir.8b01680.

[13] J. H. Snoeijer, E. Rolley, and B. Andreotti, "Paradox of contact angle selection on stretched soft solids", Physical Review Letters 121, 068003 (2018).

[14] S. Karpitschka, S. Das, M. van Gorcum, H. Perrin, B. Andreotti, and J. H. Snoeijer, "Soft wetting: Models based on energy dissipation or on force balance are equivalent", Proceedings of the National Academy of Sciences 115, E7233-E7233 (2018). 
[15] H. Liang, Z. Cao, Z. Wang, and A. V. Dobrynin, "Surface stress and surface tension in polymeric networks", ACS Macro Letters 7, 116-121 (2018), URL https://doi.org/10.1021/acsmacrolett.7b00812.

[16] R. Masurel, M. Roché, L. Limat, I. Ionescu, and J. Dervaux, "Elastocapillary ridge as a noninteger disclination", Phys. Rev. Lett. 122, 248004 (2019).

[17] R. Shuttleworth, "The surface tension of solids", Proceedings of the Physical Society. Section A 63 (1950).

[18] B. Andreotti and J. H. Snoeijer, "Soft wetting and the Shuttleworth effect, at the crossroads between thermodynamics and mechanics", EPL 113, 66001 (2016).

[19] A. Marchand, S. Das, J. H. Snoeijer, and B. Andreotti, "Capillary Pressure and Contact Line Force on a Soft Solid", Phys. Rev. Lett. 108, 094301 (2012).

[20] J. H. Weijs, B. Andreotti, and J. H. Snoeijer, "Elasto-capillarity at the nanoscale: on the coupling between elasticity and surface energy in soft solids", Soft Matter 9, 8494 (2013), URL http://xlink.rsc.org/?DOI= c3sm50861g.

[21] J. G. Kirkwood and F. P. Buff, "The Statistical Mechanical Theory of Surface Tension", The Journal of Chemical Physics 17 (1949), URL http://dx.doi.org/10.1063/1.1747248http://aip.scitation. org/toc/jcp/17/3.

[22] M. Nijmeijer, C. Bruin, A. Bakker, and J. Van Leeuwen, "Wetting and drying of an inert wall by a fluid in a molecular-dynamics simulation", Phys. Rev. A 42, 6052-6059 (1990).

[23] J. Stecki and S. Toxvaerd, "Correlations in the liquid interfaces of simple liquids", The Journal of Chemical Physics 103, 9763-9771 (1995), URL https://doi.org/10.1063/1.469940.

[24] H. Mehrabian, J. Harting, and J. H. Snoeijer, "Soft particles at a fluid interface", Soft Matter 12, 1062-1073 (2016), URL http://dx.doi.org/ 10.1039/C5SM01971K.

[25] K. Kremer and G. S. Grest, "Dynamics of entangled linear polymer melts: A molecular-dynamics simulation", The Journal of Chemical Physics 92, 5057-5086 (1990), URL https://doi.org/10.1063/1.458541. 
[26] S. Pronk, S. Páll, R. Schulz, P. Larsson, P. Bjelkmar, R. Apostolov, M. R. Shirts, J. C. Smith, P. M. Kasson, D. van der Spoel, B. Hess, and E. Lindahl, "GROMACS 4.5: a high-throughput and highly parallel open source molecular simulation toolkit", Bioinformatics 29, 845-854 (2013), URL http://www.ncbi.nlm.nih.gov/pubmed/ 23407358http://www.pubmedcentral.nih.gov/articlerender.fcgi? artid=PMC3605599https://academic.oup.com/bioinformatics/ article-lookup/doi/10.1093/bioinformatics/btt055.

[27] B. Hess, C. Kutzner, D. van der Spoel, and E. Lindahl, "GROMACS 4: Algorithms for Highly Efficient, Load-Balanced, and Scalable Molecular Simulation", Journal of Chemical Theory and Computation 4, 435-447 (2008), URL http://www.ncbi.nlm.nih.gov/pubmed/26620784http:// pubs.acs.org/doi/abs/10.1021/ct700301q.

[28] D. Van Der Spoel, E. Lindahl, B. Hess, G. Groenhof, A. E. Mark, and H. J. C. Berendsen, "GROMACS: Fast, flexible, and free", Journal of Computational Chemistry 26, 1701-1718 (2005), URL http://www.ncbi.nlm.nih.gov/pubmed/16211538http:// doi.wiley.com/10.1002/jcc.20291.

[29] W. Humphrey, A. Dalke, and K. Schulten, "VMD: Visual molecular dynamics", Journal of Molecular Graphics 14, 33 - 38 (1996), URL http: //www.sciencedirect.com/science/article/pii/0263785596000185.

[30] J. M. Vanegas, A. Torres-Sánchez, and M. Arroyo, "Importance of Force Decomposition for Local Stress Calculations in Biomembrane Molecular Simulations", Journal of Chemical Theory and Computation 10, 691-702 (2014), URL http://pubs .acs .org/doi/abs/10.1021/ct4008926.

[31] A. Torres-Sánchez, J. M. Vanegas, and M. Arroyo, "Examining the mechanical equilibrium of microscopic stresses in molecular simulations", Phys. Rev. Lett. 114, 258102 (2015), URL https://link.aps.org/doi/ 10.1103/PhysRevLett.114.258102. 


\section{Summary}

Soft polymeric solids, as inspired by nature, have gained in popularity among scientists and engineers, for their potential in technological applications. These soft solids can be engineered to respond to external stimuli, such as $\mathrm{pH}$, humidity, temperature or light. In order to truly employ all the possibilities of these soft solids, it is important to have a good understanding of the fundamental behavior of these substances on a molecular level. This thesis sheds further light and insights on the wetting behavior of soft solids, by using Molecular Dynamics simulations. In short, we explored the wetting phase transitions present for wetted polymer brushes, the dependence of their wetting transitions on both enthalpic and entropic interactions, and we explored the difference between surface free energies and surface tensions for soft solids.

In the first two chapters, we provide a general background on the wetting of soft polymeric materials, as well as on the method of Molecular Dynamics.

Then, in chapter 3, we focus on the wetting behavior of a polymer brush, wetted by a polymeric nanodroplet. We varied the interaction parameters between the brush and the droplet, and the brush-brush interactions, to increase and decrease the wettability of the substrate. We report the occurrence of three wetting states for a polymer brush-polymer melt system: mixing, complete wetting, and partial wetting. The transitions between these wetting states could largely be predicted using only enthalpy-based theories. To predict the exact location of the wetting transition however, entropic effects also needed to be taken into account. In this chapter, we also report an autophobic wetting state, which is found for melts wetting a soft brush. This autophobic wetting behavior is the opposite of what is generally found for the behavior of gels, where softer surfaces induce a transition from Young to Neumann state wetting.

In chapter 4, we continued our research on the wetting behavior of polymer brushes, focusing on the role of entropic interactions on the transitions between wetting states. We have varied the interaction parameters between brush and droplet, and between the brush particles, and we have varied the brush grafting density and the polymer melt chain length, to find their influ- 
ence on the wetting transitions. From these tests, we have shown that the transition from mixing to demixing shows a positive entropic contribution for short melt chains and low brush grafting densities, so that the transition to demixing is inhibited. For longer melt chains and higher grafting densities, the entropy contributes negatively to the transition, meaning that demixing is facilitated instead. These effects are caused due to the densely grafted polymer brushes having to pay an entropic penalty upon stretching, to allow for mixing to occur, whereas polymer melts gain translational entropy upon mixing, yet this entropy gain becomes smaller for longer polymer melt chains. For the complete to partial wetting transition, we have found that short melt chains show higher wettability, and we report only a weak influence of the grafting density upon the wetting transition. Similarly to the mixing to demixing transition, short melt chains are more likely to wet a surface completely, due to a larger gain in the translational entropy. To conclude this chapter, we have considered the partial wetting state solely, where we compared droplet contact angles to measurements of the surface tension. We found here that the measurement of surface energies from contact angle measurements, do not compare well to measurements of the surface tension from Kirkwood-Buff analysis.

Lastly, in chapter 5 , we further research the fundamental difference between surface energies and surface tensions. To do so, we switched our scope from polymer brushes to simpler systems using polymer gels and cubic crystals, so that the elastic properties will be homogeneous throughout the material. We have compared the wetting behavior of liquids wetting on strained gels and crystals, to explore the difference between the surface free energy and the surface stress in these materials. We have employed two tests of the surface tensions, using contact angles and elastic Wilhelmy plate measurements. From both measurements, we observed a clear dependency of the surface energies on the strain for the crystal, whereas no such effects were found for the polymer gel.

This thesis, in short, reports the importance of taking into account both enthalpic and entropic effects in determining what wetting states can be found for the wetting of soft substrates. We also report on ways to quantify the difference between surface free energy and surface tension in solids, where we find only minimal effects for a soft gel, whereas a rigid crystal shows clear differences. With these conclusions, this thesis opens up ideas for further research into the importance and presence of this difference, called the Shuttleworth effect, in other soft solids such as polymer brushes. As the presence of a Shuttleworth effect for soft gels is still up for debate, it is interesting to also look into the Shuttleworth effect for other types soft solids. This might give rise to new insights in the origin of the Shuttleworth effect. Especially since chapter 4 
hints at the possibility of a measurable Shuttleworth effect in polymer brushes. 


\section{Samenvatting}

Zachte vaste stoffen, zoals geïnspireerd door de natuur, hebben dankzij hun potentie voor technologische toepassingen een toename gezien in hun populariteit onder wetenschappers en ingenieurs. Deze zachte vaste stoffen kunnen zodanig bewerkt worden zodat ze reageren op externe stimuli zoals de $\mathrm{pH}$, luchtvochtigheid, temperatuur en/of licht. Om werkelijk volledig gebruik te kunnen maken van alle mogelijkheden die deze zachte vaste stoffen bieden, is het belangrijk om een goed begrip te hebben van het fundamentele gedrag van deze materialen op een moleculair niveau. Deze thesis geeft nieuwe inzichten in het bevochtigingsgedrag van deze zachte materialen, door middel van Moleculaire Dynamica simulaties. In deze thesis hebben we de wetting fasetransities verkend voor polymeerborstels, de afhankelijkheid van deze wettingtransities van zowel enthalpische als entropische interacties, en hebben we het verschil tussen de oppervlakte vrije energiën en oppervlaktespanningen verkend voor zachte vaste stoffen.

In de eerste twee hoofdstukken geven we een algemene achtergrond over de bevochtiging van zachte polymere materialen, en over de Moleculaire Dynamicamethode.

In hoofdstuk 3 focussen we op het bevochtigingsgedrag van een polymeerborstel die bevochtigd word door een polymeersmelt nanodruppel. We hebben de interactieparameters tussen de borstel en de druppel, en tussen de borstelborstel interacties gevariëerd, om zo het bevochtigingsvermogen van het substraat te verhogen en te verlagen. In onze simulaties vinden we drie bevochtigingstoestanden: mengen, complete bevochtiging, en gedeeltelijke bevochtiging. De overgangen tussen deze bevochtigingstoestanden kunnen grotendeels voorspeld worden op basis van theoriën die enkel op enthalpische interacties gebaseerd zijn. Om de exactie locatie van de bevochtigingstransitie te voorspellen, is het echter nodig om ook entropische effecten mee te nemen. In dit hoofdstuk melden we ook het bestaan van een autofobische bevochtiginstoestand, die voorkomt in gevallen waar een polymeersmelt een zachte borstel bevochtigd. Dit autofobe bevochtigingsgedrag is het tegenovergestelde van wat over het algemeen gevonden wordt voor het gedrag in polymere gels, 
waar zachtere oppervlakken een overgang veroorzaken van Young's bevochtigingstoestand naar de Neumann's bevochtigingstoestand.

In hoofdstuk 4 vervolgen we ons onderzoek naar het bevochtigingsgedrag van polymeerborstels en focussen we op de rol van entropische interacties op de overgang tussen bevochtigingstoestanden. De interactieparameters tussen de borstel en de druppel, en tussen de borsteldeeltjes zijn gevariëerd, en de borstel entdichtheid en de polymeersmelt-ketenlengte zijn gevariëerd, om zo de invloed van deze factoren te vinden op de bevochtigingsovergangen. Uit deze simulaties hebben we aangetoond dat de overgang van mengen naar ontmengen, een positieve entropische bijdrage laat zien voor korte smeltketens en lage borstel entdichtheden, wat betekent dat de overgang naar ontmengen verhinderd wordt. Voor langere smeltketens en hogere entdichtheden geeft de entropie een negatieve bijdrage aan de overang, zodat ontmenging juist vergemakkelijkt wordt. Deze effecten worden veroorzaakt doordat, door de hoge entdichtheid van de borstels, de polymeerborstels een entropische penalty betalen, doordat ze uit moeten rekken om menging van de fases toe te kunnen laten. Daarentegen verkrijgen de polymeersmeltketens translationele entropie door te mengen, maar deze entropische winst voor de polymeersmelt wordt kleiner als de smeltketens langer worden. Voor de overgang van complete naar gedeeltelijke bevochtiging, hebben we gevonden dat korte smeltketens een hogere bevochtbaarheid vertonen, en vinden we slechts een zwakke invloed van de entdichtheid op de bevochtigingsovergang. Vergelijkbaar met de argumenten voor de overgang van mengen naar ontmengen, zijn de korte smeltketens hier meer geneigd om een oppervlak compleet te bevochtigen, doordat zij een grotere winst in translationele entropie ervaren. Ter afsluiting van dit hoofdstuk, hebben we ons gericht op enkel de gedeeltelijke bevochtigingstoestand, waar we contacthoekmetingen van de druppels hebben vergeleken met metingen van de oppervlaktespanning. We hebben hier geconcludeerd dat de metingen van oppervlakte energiën door middel van contacthoekmetingen niet goed vergeleken kunnen worden met metingen van de oppervlaktespanning uit Kirkwood-Buff analyses.

Ten slotte hebben we in hoofdstuk 5 verder onderzoek verricht naar het fundamentele verschil tussen de oppervlakte-energiën en oppervlaktespanningen. Om dit verschil goed te kunnen onderzoeken, hebben we onze focus verlegd van polymeerborstels naar twee eenvoudigere systemen, polymere gels en kubische kristallen, zodat de elastische eigenschappen homogeen zullen zijn door de bulk van het materiaal. We hebben het bevochtigingsgedrag vergeleken voor vloeistoffen die aangespannen gels en kristallen bevochtigen. We hebben gebruik gemaakt van twee tests om de oppervlaktespanningen te meten, door middel van contacthoekmetingen en de elastische Wilhelmy-plaat. 
Uit beide metingen hebben we een duidelijke afhankelijk geobserveerd van de oppervlakte-energiën op de mate van spanning op het kristal, terwijl zulke effecten niet waargenomen zijn voor de polymeergel.

Kortom, in deze thesis rapporteren we het belang van zowel de enthalpische als de entropische effecten in het bepalen van welke bevochtigingstoestand verwacht kan worden bij de bevochtiging van zachte vaste stoffen. We tonen ook twee manieren om het verschil te kwantificeren tussen de oppervlakte vrije energie en oppervlaktespanning in vaste stoffen, waar we slechte minimale effecten gevonden hebben voor zachte gels, terwijl rigide kristallen duidelijke verschillen vertonen. Met hulp van deze conclusies, verschaft deze thesis ideeën voor vervolgonderzoek naar het belang en de aanwezigheid van dit verschil in de oppervlakte-energie en oppervlaktespanning, die ook wel het Shuttleworth-effect genoemd wordt, in andere zachte vaste stoffen zoals polymeerborstels. Aangezien de aanwezigheid van een Shuttleworth-effect voor zachte gels nog steeds ter discussie staat, is het interessant om ook te kijken naar de mogelijke aanwezigheid van het Shuttleworth effect in andere soorten zachte materialen. Dit zou kunnen leiden tot nieuwe inzichten in de oorsprong van het Shuttleworht-effect, vooral aangezien hoofdstuk 4 aanwijzingen geeft naar de mogelijke aanwezigheid van een waarneembaar Shuttleworth-effect in polymeerborstels. 


\section{Acknowledgements}

After 4 years, my period as a PhD-student at the Physics of Fluids group has now come to an end, and with that, also a full decade at the University of Twente. Of course, during this time, I had the privilege of getting to know a lot of people that were there to help and guide me. Hereby, I would like to thank all of you.

First off, I would like to thank my supervisors, Jacco and Sissi. I cannot express how much optimism and fascination with science have been of help to me. Jacco, thank you for explaining the concepts of wetting, elasticity, and general mathematical formulations many times. You were always patient with me and have been of help numerous times. Thank you for trusting me with this research. Sissi, thank you for introducing me to the world of computer simulations. I truly believe that these simulations form a good addition to experimental research, due to their controlled environments. I would also like to thank you for your unparalleled enthusiasm and fascination with polymeric research. Whenever I came up with results that I was not initially able to explain, I could always count on your reaction to be 'Cool! Let's get to the bottom of this!'. You have been a true inspiration to me.

Besides my supervisors, I want to thank Hadi, who has helped me out during the first few months of my PhD. Thank you for giving me advice on all the computational aspects of my computer simulations, your have helped me out a lot in those few months.

I want to thank the supporting staff at our group. Bas, thank you for all the times that you have helped me to get my computer back on the network, and for solving all my LINUX and cluster-related problems. Joanita, thank you for all that you do for this group. You are always fast and efficient. I do not know what PoF would do without you. Gert-Wim, although I may not have needed your help often, you were always friendly and I do very much appreciate having fellow Tukkers around.

I would also like to thank my officemates who have kept me company during many hours in the office. Alvaro, thank you for being there with me for almost my entire journey. To this day, I still miss your DJ skills and bright demeanor in 
the office. Minori, thank you for your company in the office. I really enjoyed our conversations about the Japanese culture. Maxime, you were mischievous, yet always lovable. Thank you for making my desk look well-organized. Michiel, thank you for your company and all our conversations about politics and human rights. It is always nice to have a fellow-socialist/environmentalist in my presence. Pablo, it's a pity we have not been able to talk more during your first few months and my last few months here. Nevertheless, you were friendly company!

Now, I would like to thank my paranymphs, Myrthe and Simone. Myrthe, we already knew each other before we both started working at PoF. I really admire the way that you can communicate so openly with people. You were always up for a much-needed tea break and offered a listening ear when needed. Simone, you joined me on the adventure of pursuing a $\mathrm{PhD}$ at a physics group, whilst having a background in chemistry. We may not have been part of the same research group, but you were never far, and so we were able to catch up over a drink. I want to thank you both for your friendship and for being my paranymphs.

I would also like to give thanks to my colleagues. PoF is a large group, with ample opportunities to discuss science, but also for social interactions and sports. I want to thank our little BATA running club. Thank you to Jessica and Anja for undertaking this adventure with me of 'learning how to run', and thank you to Myrthe and Maaike for joining us and for giving us extra motivation to keep running. Running definitely became more fun with your company. I had a great time at the BATA thanks to you all. During my last months here, I also took up bouldering. I would like to thank our group of PoF-climbers for their company during my time in the boulder hall. Thank you all for teaching me how to succesfully climb up some simulated mountains, Ricardo, Maaike, Jessica, Carola, Rodrigo, Pierre, Dennis and Biljana! I would also like to thank all the people that joined for our many walks during lunch time. Thank you for your company and for the good conversations. We have had frequent walks together, Rianne, Pascal, Michiel, Arjen, Robin, Kirsten, Maaike, Myrthe, Ruben, Michiel, Jessica and Anja.

I also want to thank Sjoukje, for her help on designing my thesis cover.

Dan wil ik ten slotte ook graag mijn vrienden en mijn familie bedanken voor hun aanwezigheid, interesse en steun. Jullie betekenen ongelooflijk veel voor mij. Mam, pap en Juri, bedankt voor alles wat jullie voor me doen. Bedankt dat jullie altijd klaarstaan om te luisteren, en langs te komen als ik het moeilijk heb. Bedankt voor jullie interesse in mijn onderzoek en alle steun die ik van jullie heb mogen ontvangen gedurende mijn leven.

Gerwin, bedankt voor alles dat je me geeft. Ik beleef graag samen met jou 
de avonturen die het leven te bieden heeft, zowel groot als klein. Het is een voorrecht om met jou te mogen zijn. Bedankt voor je aanwezigheid en al je steun en aandacht. 
